\title{
Enhanced Molecular Dynamics Approach for Thermal Transport Phenomena in Complex Systems
}

\author{
by
}

\section{Sylvie Antoun}

M.Sc in Mechanical Engineering, American University of Beirut, 2015

B.Sc in Mechanical Engineering, American University of Beirut, 2014

\author{
A dissertation \\ presented to Ryerson University \\ in partial fulfillment of the \\ requirements for the degree of \\ Doctor of Philosophy \\ in the Program of \\ Mechanical and Industrial Engineering
}

Toronto, Ontario, Canada, 2019

(C)Sylvie Antoun 2019 


\section{AUTHOR'S DECLARATION FOR ELECTRONIC SUBMISSION OF A DISSERTATION}

I hereby declare that I am the sole author of this dissertation. This is a true copy of the dissertation, including any required final revisions, as accepted by my examiners.

I authorize Ryerson University to lend this dissertation to other institutions or individuals for the purpose of scholarly research.

I further authorize Ryerson University to reproduce this dissertation by photocopying or by other means, in total or in part, at the request of other institutions or individuals for the purpose of scholarly research.

I understand that my dissertation may be made electronically available to the public. 


\title{
Abstract
}

\author{
Enhanced Molecular Dynamics Approach for Thermal Transport Phenomena in Complex \\ Systems \\ Doctor of Philosophy 2019 \\ Sylvie Antoun \\ Mechanical and Industrial Engineering \\ Ryerson University
}

This thesis introduces an enhanced Molecular Dynamics (MD) approach, blended with fine-tuned Force Field (FF) models to reflect more realistic experimental conditions and achieve a precise representation of the atomic interactions in complex systems.

Firstly, an enhanced MD algorithm consisting of an upgraded non-equilibrium integration scheme, namely eHEX, coupled with an augmented TraPPE-UA force field, was generated and put to use to predict Soret effect in a binary mixture: n-pentane/n-decane. The results were compared to other MD approaches and validated with respect to benchmarked experimental data. The suggested method showed a closer agreement with experimental data than the previous MD findings. The reinforced potential field (TraPPE-UA) was capable of reflecting the real molecular interactions between the hydrocarbons and reproduce the liquid mixture properties at different conditions. Moreover, the extended HEX method succeeded in conserving the system's overall energy with minor fluctuations and attaining a stationary state, ensuring the precision of the integration scheme and the satisfaction of local equilibrium.

Secondly, the performance of the previously proposed approach was further studied to test its performance on a ternary mixture of methane/n-butane/n-dodecane at five different compositions. Thermodiffusion separation ratio of each component was assessed at 333.15 $\mathrm{K}$ and $35 \mathrm{MPa}$, and compared to the experimental data as well as 3 other MD models from the literature. A good qualitative agreement between the experimental data and the MD model observed in this work was observed, displaying the least deviation when compared to the other MD approaches. The method was capable of adequately representing the physics

behind the thermodiffusive separation and deepening the microscopic understanding of the segregation process in a ternary mixture undergoing large thermal gradients. Put differently, the approach elucidates the relative contribution of the cross-interactions found between the unlike species in the mixture and their corresponding composition.

Next, an enhanced MD approach was also presented to predict the dynamics and thermophysical properties of suspended $\gamma$-alumina nanoparticles (NPs) in acidic aqueous solutions. The previous MD work have unveiled numerous impediments in terms of reproducing the thermal transport phenomena in nanofluids. A hybrid potential field, comprised of refined 
force field models (ClayFF and SPC/E), was implemented to allow a precise integration of the nanoscale phenomena into the dynamics and structure of charged alumina NPs, thereby bridging the challenging gap between the solid-liquid interfacial chemistry and the overall thermodynamic properties. The original CLAYFF was augmented to properly account for the energy and momentum transfer between the water molecules and the positively charged NPs, while keeping the number of parameters small enough to allow modeling of a relatively large nanofluidic system. The results were in good agreement with the experimental data. An increase of the NPs volumetric concentration $(\phi)$ lead to the enhancement of thermal conductivity along with an increase of viscosity. The results demonstrate the crucial role played by the repulsive electrostatic forces yielding well-dispersed NP suspensions, specially at low $\phi$. The post analysis of Mouromtseff number demonstrated that at lower $\phi$, the system show a higher propensity for stability and enhancement for $\phi$ less than $2 \%$, specially at high temperatures. On the contrary, for volumetric concentrations higher than $2 \%$, the system thermal performance deteriorates which is expected due to the fact that the system exhibit a critical condition of aggregation and clogging.

With all of the above findings in mind, the MD framework presented in this thesis represents an improved step towards a precise and computationally balanced MD modelling that bridges the relation between molecular signatures and macroscopic features, capable of overcoming the shortcomings present in mainly two emerging thermal applications: 1) Soret effect in hydrocarbon mixture and 2) thermal transport of alumina-water nanofluids.

Thesis Supervisor: M. Ziad Saghir

Thesis Co-Supervisor: Seshasai Srinivasan

Title: Professor, Department of Mechanical Engineering 


\section{Acknowledgements}

In handing in this dissertation, I wish to express my gratitude for the people whose support was instrumental in completing my $\mathrm{PhD}$ at Ryerson University.

First, I would like to kindly thank my advisor, Prof. M. Ziad Saghir, who provided me with the opportunity to come to Canada and pursue my research. His guidance and his trust are greatly appreciated. He has also offered candid career advice which will be invaluable in shaping my future. I also would like to thank Prof. Seshasai Srinivasan for his academic guidance and for co-advising me throughout my PhD journey.

Secondly, my engagement with the Ryerson community was enriched by the wonderful people I met, especially at the Athletic Department. Among those, I would like to single out Meghan Gaudette and Pippin Lee, for being my closest friends and supporting me through everything. Our conversations, laughter and wild adventures carried me through many crazy weeks.

Most importantly, I want to express my deepest gratitude to the man I met during my PhD journey, Xavier Yuvens Lachance. He helped me reconnect with my initial fire, realize the importance of a balanced life and push through all the challenges I faced, with a smile on my face. It is hard for me to imagine completing this dissertation without his presence by my side; he was the greatest inspiration.

Finally, this picture wouldn't be complete without acknowledging the unwavering support and encouragements of my mom, Suzanne, my dad, Jacques and my sisters, Maya and Sally. Their faith, care and love for me give meaning to my achievements. 


\section{Table of Contents}

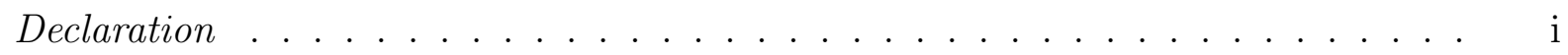

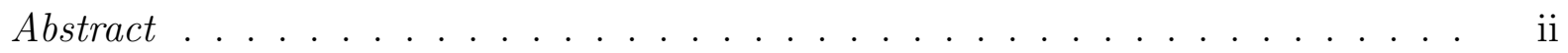

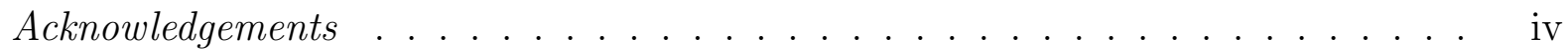

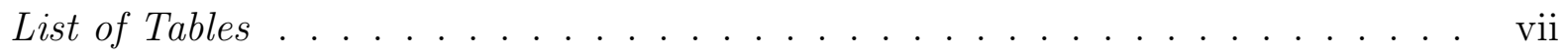

List of Figures ............................. ix

$\begin{array}{llr}1 & \text { Introduction } & 1\end{array}$

1.1 Project Motivation . . . . . . . . . . . . . . . . . . . . . . 3

1.2 Objective and Scope $\ldots \ldots \ldots \ldots \ldots \ldots$

1.3 Thesis structure $\ldots \ldots \ldots \ldots \ldots \ldots \ldots$

\begin{tabular}{lll}
\hline 2 & Applications Background & 10
\end{tabular}

2.1 Thermodiffusion in Petroleum . . . . . . . . . . . . . . . . . . 10

2.2 Thermal Performance in Nanofluids . . . . . . . . . . . . . . . . . 12

3 Molecular Dynamics: Methodology, Applications, and Perspectives 15

3.1 Foundation . . . . . . . . . . . . . . . . . . . . . 15

3.2 Force Field Models . . . . . . . . . . . . . . . . . . . . . . . . . 18

3.3 Linear Response Theory: Heat and Mass Transport . . . . . . . . . . . . . . 21

3.4 MD Algorithms for Transport Coefficients Calculation. . . . . . . . . . . . 25

$3.4 .1 \quad$ Equilibrium Molecular Dynamics (EMD) . . . . . . . . . . . . 25

3.4 .2 Non-Equilibrium Molecular Dynamics (NEMD) . . . . . . . . . . . 26

4 Enhanced Molecular Dynamics Modeling of Thermodiffusion in Binary $\begin{array}{lr}\text { Mixtures } & 29\end{array}$

$4.1 \quad$ Introduction . . . . . . . . . . . . . . . . . . . . . . . . . . . . . . . . . . . . . . 29 
4.2 Theoretical Formulation . . . . . . . . . . . . . . . . . . . . . . 34

4.2 .1 The Enhanced Heat Exchange (eHEX) Algorithm . . . . . . . . . . . 34

4.2 .2 Force Field Model . . . . . . . . . . . . . . . . . . . . . . . . . . 36

4.3 Methodology and Numerical Computations . . . . . . . . . . . . . . . 38

4.3 .1 EMD Simulations . . . . . . . . . . . . . . . . . . . . . . . . . . . . . . 38

4.3 .2 BD-NEMD Simulations . . . . . . . . . . . . . . . . . . . . . . 40

4.4 Results and Discussions $\ldots \ldots \ldots$. . . . . . . . . . . . . . . . . . . . . 41

4.4 .1 Validation of the Force Field Model . . . . . . . . . . . . . . . 41

4.4 .2 Linearity and Convergence of Composition and temperature profiles . 42

4.4 .3 Comparison of $S_{T}$ with experimental and other numerical work . . 49

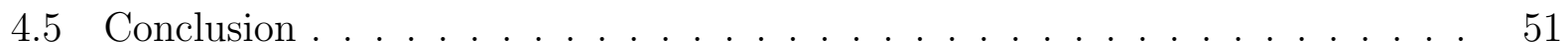

5 Enhanced Molecular Dynamics Evaluation of Thermophobicity in Ternary $\begin{array}{ll}\text { Mixtures } & 54\end{array}$

5.1 Introduction . . . . . . . . . . . . . . . . . . . . 54

5.2 Methodology . . . . . . . . . . . . . . . . . . . 57

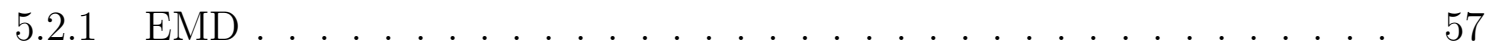

5.2 .2 BD-NEMD $\ldots \ldots \ldots \ldots \ldots \ldots \ldots$

5.3 Results and Discussion . . . . . . . . . . . . . . . . . . . . . . . . . . 59

$5.3 .1 \quad$ Validation of mixture density and self-diffusion coefficient . . . . . . 59

5.3 .2 Thermodiffusion Factor $\ldots \ldots \ldots \ldots$

5.4 Conclusion . . . . . . . . . . . . . . . . . . . . . . . . . 71

6 Molecular Dynamics Evaluation of Alumina Nanofluid Thermal Transport Performance $\quad 73$

6.1 Introduction . . . . . . . . . . . . . . . . . . . . . . . . . 73

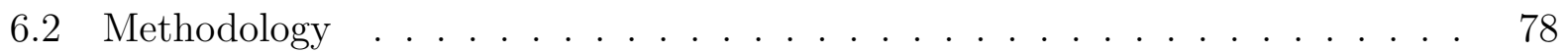

$6.2 .1 \quad$ Initial Configuration and Choice of Potential FF . . . . . . . . . . 79

6.2 .2 EMD Simulations . . . . . . . . . . . . . . . . . . . . . . . . 82

6.3 Results and Discussions $\ldots \ldots$. . . . . . . . . . . . . . . . . . . . . . 83

6.3 .1 Simulation Model Validation . . . . . . . . . . . . . . . . . . . . 83

6.3 .2 Thermal conductivity and Viscosity calculation . . . . . . . . 85

6.3 .3 Mouromtseff Factor $(\mathrm{Mo}) \ldots \ldots \ldots$. . . . . . . . . . . . 88 
6.4 Conclusion . . . . . . . . . . . . . . . . . . . . . . . . 93

7 Conclusions and Future Research Directions 95

7.1 Contributions . . . . . . . . . . . . . . . . . . . 95

7.1.1 Thermodiffusion in Binary n-alkanes . . . . . . . . . . . . . . . . . . 95

7.1 .2 Thermodiffusion in Ternary n-alkanes . . . . . . . . . . . . . . . . . . 96

7.1.3 Thermal Transport of Nanofluids . . . . . . . . . . . . . . . . . . . . 97

7.2 Challenges Faced $\ldots \ldots \ldots$. . . . . . . . . . . . . . . . . . . . . . . . . . . . . . 98

7.3 Recommendations for Future Work . . . . . . . . . . . . . . . . . . . . . . . 99

A Molecular Dynamics Evaluation of Alumina Nanofluid Thermal Transport $\begin{array}{lr}\text { Performance } & 101\end{array}$

\begin{tabular}{ll}
\hline Bibliography & 115
\end{tabular} 


\section{List of Tables}

4.1 Potential energy functions and parameters for the TraPPE UA model . . . . 37

4.2 Densities of $\mathrm{nC}_{5}-\mathrm{nC}_{10}$ mixture for different molar composition at $300.15 \mathrm{~K}$ and $0.1 \mathrm{MPa}$. The $\%$ error $\Delta$ of the numerical density of this work was computed with respect to NIST data . . . . . . . . . . . . . . . . . . . . . . 42

4.3 Potential energy functions and parameters for the TraPPE UA model . . . . 42

4.4 Experimental data of diffusion $(D)$ and thermal diffusion coefficients $\left(D_{T}\right)$ measured using different techniques at $300.15 \mathrm{~K}$ for the $\mathrm{nC}_{5}-\mathrm{nC}_{10}$ mixture for three different molar compositions. . . . . . . . . . . . . . . . . . . . . 49

4.5 Numerical values of ST computed using BD-NEMD with different force field (FF) models for the the $\mathrm{nC}_{5}-\mathrm{nC}_{10}$ mixture at three different molar compositions. The values in the brackets represent the percentage error with respect to the benchmark values. HEX along with augmented TraPPE-UA* correspond to this work results. . . . . . . . . . . . . . . . . . . . . . . . 51

4.6 Numerical values of $S_{T}$ computed using HEX and eHEX along with augmented TraPPE-UA * for the $\mathrm{nC}_{5}-\mathrm{nC}_{10}$ mixture at three different molar compositions. The values in the brackets represent the percentage error with respect to the benchmark values. . . . . . . . . . . . . . . . . . . . . . . 52

5.1 Densities of $\mathrm{nC}_{1}-\mathrm{nC}_{4}-\mathrm{nC}_{12}$ mixture for different molar composition at 333.15 $\mathrm{K}$ and $35 \mathrm{MPa}$. The \% error $\Delta$ of the numerical density of this work was computed with respect to experimental data. . . . . . . . . . . . . . . . 60

5.2 Self-diffusion coefficients $(D)$ of $\mathrm{nC}_{1}, \mathrm{nC}_{4}$ and $\mathrm{nC}_{12}$ for the different ternary mixture molar compositions. . . . . . . . . . . . . . . . . . . . . . . 61 
5.3 Numerical values of thermodiffusion factor computed for each component using this work MD algorithm and other models of Galliero [1] for all the molar compositions. The values in brackets represent the percentage error with respect to the experimental values. . . . . . . . . . . . . . . . . . . 67

5.4 Experimental and this work MD results of thermodiffusion separation ratio $k_{T, i}^{\prime}$ for each component at every mixture mole fraction. . . . . . . . . . . . . 70

A.1 Force field parameters implemented in this work [2, 3] . . . . . . . . . . . . . 102 


\section{List of Figures}

4.1 Illustration of the simulation box, $\Omega$, with a hot reservoir, $\Gamma_{1}$ (red), and a cold reservoir, $\Gamma_{2}$ (blue). The center of mass velocities of $\Omega, \Gamma_{1}$, and $\Gamma_{2}$ are $v_{\Omega}$, $v_{\Gamma 1}$, and $v_{\Gamma 2}$, respectively. . . . . . . . . . . . . . . . . . . . . . 35

4.2 Temperature (top), density (middle), and $\mathrm{nC}_{10}$ mole fraction (bottom) profiles for the three different molar compositions of $\mathrm{nC}_{5}-\mathrm{nC}_{10}$ : (a) $x_{C 10}=0.2$, (b) $x_{C 10}=0.5$, and (c) $x_{C 10}=0.8 \ldots \ldots$. . . . . . . . . . . 43

$4.3 \quad$ System overall temperature (red) and energy (blue) variation as a function of simulation time for the three different molar compositions of $\mathrm{nC}_{5}-\mathrm{nC}_{10}$ : (a) $x_{C 10}=0.2$, (b) $x_{C 10}=0.5$, and (c) $x_{C 10}=0.8$. . . . . . . . . . . . . . . 45

4.4 Temperature (top) and $\mathrm{nC}_{10}$ mole fraction (bottom) profiles for the three different molar compositions of $\mathrm{nC}_{5}-\mathrm{nC}_{10}$ : (a) $x_{C 10}=0.2$, (b) $x_{C 10}=0.5$, and (c) $x_{C 10}=0.8$. . . . . . . . . . . . . . . . . . . . . . . . . 46

4.5 System density profile for the three different molar compositions of $\mathrm{nC}_{5}-\mathrm{nC}_{10}$ : (a) $x_{C 10}=0.2$, (b) $x_{C 10}=0.5$, and (c) $x_{C 10}=0.8$. . . . . . . . . . . . . 47

4.6 Temperature (top) and number density of $\mathrm{nC}_{10}$ (bottom) gradients variation over simulation time for the three different molar compositions of $\mathrm{nC}_{5}-\mathrm{nC}_{10}$ : (a) $x_{C 10}=0.2$, (b) $x_{C 10}=0.5$, and (c) $x_{C 10}=0.8$. . . . . . . . . . . . . 48

5.1 Normalized local kinetic energy (left) and potential energy (right) variation with NEMD simulation time in hot (red), cold (blue), and middle (green) slab regions and represented for (a) mixture $2\left(\mathrm{xC}_{4}=0.2\right)$, (b) mixture $3\left(\mathrm{xC}_{4}=\right.$ $0.4)$, and (c) mixture $5\left(\mathrm{xC}_{4}=0.6\right) . \ldots \ldots \ldots$. . . . . . . . 63 
5.2 Mole fraction profile of nC1 (black dots), $\mathrm{nC} 4$ (red triangles), and nC12 (blue squares) for three different molar compositions (a) mixture $2\left(\mathrm{xC}_{4}=0.2\right)$, (b) mixture $3\left(\mathrm{xC}_{4}=0.4\right)$, and (c) mixture $4\left(\mathrm{xC}_{4}=0.5\right)$. The error bars which represent the standard deviation of the mean are only shown for mixture 3 . 64

5.3 Normalized concentration (density number) gradient variation with NEMD simulation time for each component in (a) mixture $2\left(\mathrm{xC}_{4}=0.2\right)$, (b) mixture $3\left(\mathrm{xC}_{4}=0.4\right),(\mathrm{c})$ mixture $4\left(\mathrm{xC}_{4}=0.5\right)$, and $(\mathrm{d})$ mixture $5\left(\mathrm{xC}_{4}=0.6 . \quad\right.$. . 65

5.4 Comparison of the thermodiffusion factor for each component (a) $\mathrm{nC}_{1}$, (b) $\mathrm{nC}_{4}$, and (c) $\mathrm{nC}_{12}$ in the ternary mixture for all the five molar compositions. Values correspond to the experimental other numerical values in the literature and this work MD results. . . . . . . . . . . . . . . . . . . . . . . . 68

6.1 NPs dispersion morphology in water for (a) $\phi=1 \%$ (b) $\phi=2 \%$ (c) $\phi=3 \%$ (d) $\phi=4 \%$. Red and green represent oxygen and aluminum atoms of NPs and blue symbolizes water molecules (base fluid). . . . . . . . . . . . . . . . . . . 80

6.2 Variation of $\mathrm{Al}_{2} \mathrm{O}_{3}$-water nanofluid density with temperature for the different NPs volumetric concentration. . . . . . . . . . . . . . . . . . . 84

6.3 Variation of $\mathrm{Al}_{2} \mathrm{O}_{3}$-water nanofluid specific heat capacity with temperature for the different NPS volumetric concentration. . . . . . . . . . . . . . . . . . 85

6.4 Time evolution of (a) HCACF and (b) SACF for the nanofluids at $\phi=2 \%$ and $\mathrm{T}=25{ }^{\circ} \mathrm{C}$. . . . . . . . . . . . . . . . . . . 86

6.5 Variation of $\mathrm{Al}_{2} \mathrm{O}_{3}$-water nanofluid viscosity with temperature for the different NPs volumetric concentration. . . . . . . . . . . . . . . . . . . . 87

6.6 Variation of $\mathrm{Al}_{2} \mathrm{O}_{3}$-water nanofluid thermal conductivity with temperature for the different NPs volumetric concentration. . . . . . . . . . . . . . . . . . . . 88

6.7 Relative change of nanofluid viscosity (left) and thermal conductivity (right) with respect to $\phi$ at different system temperatures. . . . . . . . . . . . . . . 89

6.8 Heat Transfer efficiency ratio $\left(\mathrm{Mo}^{*}\right)$ and the corresponding thermal conductivity $\left(k^{*}\right)$ and viscosity $\left(\mu^{*}\right)$, ratios as a function of temperature for (a) $\phi=$ $1 \%$ (b) $\phi=2 \%$ (c) $\phi=3 \%$ (d) $\phi=4 \%$. . . . . . . . . . . . . . . . . . . 90

6.9 Global illustration of the heat transfer enhancement ratio computed at different $\phi(\%)$ and $\mathrm{T}\left({ }^{\circ} \mathrm{C}\right)$ conditions. . . . . . . . . . . . . . . . . . . . . 92 
A.1 Variation of the nanofluid viscosity ratio (left) and thermal conductivity ratio (right) as a function of $\phi$ for $\mathrm{T}=25^{\circ} \mathrm{C}$. . . . . . . . . . . . . . . . . 103 


\section{Chapter 1}

\section{Introduction}

This thesis is a concerted effort into developing fine-tuned molecular dynamics (MD) algorithms blended with augmented force field models in an attempt to achieve a better microscopic representation of the real atomic topologies and potential interactions, which highly affect the accuracy of thermal transport predictions. The MD models were revamped and applied to two specific applications covered in-depth throughout this dissertation work:

(1) Soret effect of multicomponent hydrocarbon mixtures: The numerical target behind this field is to unravel the pitfalls found in thermodiffusion experimental discoveries and theoretical formulations, thereby bringing a possible numerical alternative, a lowcost reliable MD tool, to complement and delineate strategies to advance the grading of pre-exploitation of hydrocarbons in underground oil reservoirs.

(2) Thermophysical and transport characteristics of alumina-water nanofluids suspended in a low $\mathrm{pH}$ level regime. The aim of this MD application is to bring a more precise image of the inter-particle bonding and dynamics with the focus on modulating the solid-liquid interface structure and charge corresponding to an acidic $\mathrm{pH}$ conditions, at which one can avoid aggregation and prolong nanofluids stability. Many theoretical and experimental efforts have been carried out to gain insight into the water metal oxide interfacial behaviour. Recent experimental findings on interfacial phenomena shed light on the specific factors influencing the inter-NP and water-NP interactions, thereby clarifying the multiple, closely coupled processes controlling the dispersion 
of NPs in water (i.e. dissolution, adsorption, protonation, hydroxylation, etc.).The MD algorithm was linked to a hybrid potential field, comprised of refined force field models (extended CLAYFF and SPC/E) to accurately reproduce the interaction potential of protonated aluminium-oxides, including correctly capturing the attractive van der Waals and the repulsive electrostatic interactions, particularly important at the nanoscale.

This enhanced MD approach stands as a promising strategy to build a rational understanding and control of NPs aggregation in the base fluid, visualize their dynamics and predict their macsrocopic properties. Moreover, to estimate the overall performance of the nanofluid in convective heat transfer applications, the predicted thermophysical properties were combined into one factor of merit, evaluated at different volumetric concentrations and temperatures. Most modern systems involve heterogeneous composition of species with dissimilar shape, size, chemical activity, and atomic interactions (such as electrostatic, dispersion and interfacial interactions). The use of more than one component introduces new variables, which strongly modify the atomic interactions and the system dynamics via cross-coupling effects. Thermal, electric and chemical effects are often present and intimately interconnected, leading to a broad spectrum of new phenomena for which a good theoretical understanding and accurate models are often lacking.

Overall, the contribution attempts of this work is to build on recent MD progress in the field of thermodiffusion and thermal transport prediction of nanofluids. On one part, the close agreement of Soret effect results with benchmarked data herein imply that our MD approach is a low-cost precise alternative and altogether a valuable link between thermodiffusion experiments and theories, essentially in oil and gas industrial applications. On the other part, the MD predictions have shown that the addition of nanoparticles (NPs) leads to a relative enhancement of the nanofluids thermal conductivity accompanied with a gain of viscosity, thereby showing a good agreement with the experimental findings [4]. The radial distribution functions along with the mean square displacement (self-diffusion) of both species, alumina NPs and the water molecules attained at each $\phi$ level, demonstrate the crucial role played by the repulsive electrostatic forces yielding well-dispersed NP suspensions, especially at low volumetric concentration (depicting a relatively lower viscosity). In fact, the 
surface charge density have shown great potential to stabilize NPs assembly in a base fluid, as demonstrated in some reported experimental papers [5], thereby qualitatively validating our modelling approach. The post analysis of the factor of merit Mouromtseff number (Mo) demonstrated that at lower $\phi$, the system show a higher propensity for enhancement and stability, specially at high temperatures. On the contrary, for $\phi$ higher than $2 \%$, the system thermal performance deteriorates, due to the fact that the nanofluid exhibits a critical condition of aggregation and clogging, thereby establishing a threshold for its functionality. The results have shown that the MD model developed here demonstrates an improved numerical effort, compared to previous MD attempts, for predicting the thermophysical properties of alumina/water while establishing a precise representation of the atomic topology and interfacial potential interactions.

An empirical-numerical synergy will eventually allow scientists to discover unique transport phenomena and open up a wide range of innovative solutions for nanofluid based thermal management, storages and convective heat transfer to design, simulate and optimize thermal systems efficiently, with a better accuracy and and computational efficiency.

The majority of the material that appears in this thesis has been either submitted for publication or published in peer-reviewed journals. This introductory chapter elaborates on the motivation, novelty, objective, scope, structure and contributions of this thesis.

\subsection{Project Motivation}

The shortfall of reliable MD tools capable of quantifying Soret effect prompted our initial drive to elucidate the underlying physics, on an atomic scale, behind the compositional grading in multicomponent mixtures under geothermal gradients and ultimately bolster the evaluation of pre-exploitation distribution of hydrocarbons in underground oil reservoirs. In particular, this interest in this work is stirred by the need to generate precise non-equilibrium MD models adequate enough to mimic thermodiffusion phenomena in underground petroleum and eventually complement/interpret the empirical database obtained from microgravity experiments conducted in space for ternary mixtures at high pressures and temperatures. 
The research gaps on both numerical and experimental sides, carried us to further investigate the below shortcomings of Soret effect quantification:

- The scarcity and lack of consensus on the available experimental data. In fact, performing thermodiffusion measurements under laboratory conditions is very sensitive and data can be easily distorted by mechanical disturbances: the relative magnitude of the components separation under the effect of a temperature gradient is very small and it is easily affected by external factors such as gravity field, mechanical vibrations and the procedure of post-processing the system components.

- Theories fail to quantify the thermodiffusive separation ratio in multicomponent asymmetric systems (entailing more than 2 components) and to account for the interplay of multiple factors such as: molecular masses, effective molecule size, temperature, mixture composition, and cross intermolecular interactions between unlike species.

- Non-Equilibrium MD (NEMD) predictions of transport properties are highly susceptible to the choice and the degree of refinement of the potential field models. The all-atomic representation of the interactions found in large systems of n-alkanes is computationally expensive. Therefore, one should find a balance between the level of resolution and computational efficiency of the force field model (i.e. reduce the total number of interactions sites and ultimately the total simulation time while still holding a precise picture of realistic inter-connections between the different hydrocarbons).

Moving on to nanofluids MD application, the numerical findings on nanofluids existing in the literature are skewed by many simplifying assumptions: they mostly neglect the effect of NPs surface atomic structure and electrostatics resulting from various chemical/physical processes occurring from solvating solid nonmaterial in aqueous solutions of different ionic strength. For example, dissolution, adsorption (physisorption and chemisorption), protonation/deprotonation and other processes highly affect local functionalities of the nanofluid found at the interface. The specific motives that brought this research to tackle MD modelling of nanofluids are the below:

- The suspension of NPs in aqueous solutions, known as nanofluid, hold promise in many energy applications, including thermal management, energy storages, sustainable power harvesting for cooling/heating purposes, etc. The ability to tune NP interactions, and 
thereby modulate the dispersion level, holds the key to rationally synthesize a stable nanofluid and extend the shelf-life of its properties and functionality. However, engineering nano-sized suspensions remain a challenging task both experimentally and theoretically. Recent empirical and quantum studies on interfacial phenomena shed light on the specific factors influencing the inter-NP and water-NP interactions, thereby clarifying the multiple, closely coupled processes controlling the interactions between NPs dispersed in water (i.e. dissolution, adsorption, protonation, hydroxylation, etc.).

- Despite the widespread experimental investigations of heat transfer in nanofluids, it remains unclear how the acidic - basic properties of alumina NPs predominantly governed by the nature and concentration of hydroxyl groups evolve in aqueous medium and affect the system macroscopic properties.

- There is an essential need to combine MD modelling with precise potential fields qualified to emulate the hydrated/protonated state of aluminum-oxide surface to help provide an improved understanding of the surface charge effect on the NPs dispersion level and the system thermal transport properties.

\subsection{Objective and Scope}

The main objective of this work is to produce enhanced MD simulations synergistically combined with augmented force field models, rectify the atomic representation of crossinteractions between the system species, and ultimately improve MD numerical predictions of thermal transport properties. This thesis thoroughly tackles two distinctive application fields: 1) Soret effect in petroleum and 2) heat transfer enhancement in nanofluids.

Briefly, the main contributions that this work offers to the research are the following:

- An enhanced non-equilibrium MD approach, namely eHEX combined with an augmented TraPPE-UA force field, was capable to adequately represent the physics behind the thermodiffusive separation (magnitude and sign) and deepen the microscopic understanding of the segregation process in binary/ternary hydrocarbon mixtures undergoing large thermal gradients.

- The ability to numerically estimate self-diffusion and thermal diffusion properties of oil reservoirs while ensuring the continuity of both the temperature and concentration 
gradients at stationary state and emphasizing that the system behavior, despite the extreme thermal fluxes, is still in the domain of linear response and converges with minimal computational error.

The proposed NEMD approach showed the closest agreement to the benchmarked experimental data compared to other MD models found in the literature. The method succeeded to conserve the system overall energy with minor fluctuations and attain a stationary state under large thermal gradients which demonstrates the precision of the integration scheme and the satisfaction of local equilibrium condition. Moreover, the reinforced potential field was capable to reflect the real molecular interactions between the hydrocarbons and reproduce the liquid mixture properties at different conditions.

In the ensuing chapters of this thesis work, we intend to produce enhanced MD simulations synergistically combined with fortified force field models (namely CLAYFF coupled with $\mathrm{SPC} / \mathrm{E}$ ), to accurately predict the thermophysical properties of alumina-water nanofluids at different volumetric concentrations and temperatures, mainly in an acidic regime ( $\mathrm{pH}$ range $3 \sim 3.75<<$ PZE) and subsequently estimate their overall performance for convective heat transfer applications.

More specifically, the aim is to develop an improved MD model capable of predicting the dynamic behavior of positively charged alumina NPs scattered in water to uncover the critical links between nanoscale and macroscale phenomena, and facilitate a controlled suspension of the nanofluid. The main objectives of the nanofluid MD application are the following:

- Forge a rational understanding and control of the interparticle potential (i.e. crossinteractions between $\mathrm{Np}$-Np, Np-water and water-water) to ensure a reliable prediction of the topology and the potential energies between the dispersed NPs and the base fluid. In fact, various chemical phenomena occur at the solid-liquid interface when immersing NPs in water, such as hydration, dissolution, protonation/deprotonation (depending on the solution ionic strength) which highly modify the surface atomic structure, partial charges, bonding types and polarity at the solid-liquid interface.

- Modulate the dispersion level of the nano-colloidal system by manipulating the solidliquid interfacial chemistry (alumina-water) to mimic the conditions of a stable system. Preventing aggregation, clogging and sedimentation of NPs in the base fluid while achieving a better enhancement of the overall thermophysical properties have been the 
primary focus of advanced nanofluids preparation methods in order to maintain their stability and extend the shelf-life of their functionality in various thermal applications [6].

In summary, our MD computations were designed to fill the gap and justify the discrepancies of the experimental and theoretical findings, by elucidating the actual thermal transport mechanisms at a hitherto unobservable scale. All in all, this thesis intends to demonstrate that an enhanced MD tool combined with fine-tuned force field models is a viable and altogether effective approach to solving complex thermal engineering problems, ultimately capable of avoiding many of the pitfalls associated with conventional theoretical and empirical frameworks.

\subsection{Thesis structure}

In this section, we briefly summarize the work flow of this dissertation. Firstly, we produce an enhanced non-equilibrium MD algorithm and implement it to predict Soret effect in a hydrocarbon binary mixture: n-pentane/n-decane. Using the same basis, we move on to a ternary mixture to gain a better insight of the thermodiffusive behavior of methane/nbutane/n-dodecane and ultimately prove the power of our enhanced method. Finally, our sights on a more energy efficient future drive our MD work towards nanofluids, specifically the evaluation of thermal transport behavior in alumina-water. The specifics of each chapter of this thesis proceed as follows:

Chapter 2 - Applications Background: This preliminary chapter introduces a brief background of the two engineering areas of interest to further contextualize and motivate the necessity of implementing enhanced MD techniques, starting with 1) thermodiffusion in hydrocarbon mixtures, and followed by 2) thermal transport of nanofluids for energy efficiency applications.

\section{Chapter 3 - Molecular Dynamics: Methodology, Applications, and Perspectives:}

This chapter discusses the fundamentals of MD simulations, including the underlying algorithms and theory, force field model and limitations. 
Chapter 4 - Enhanced Molecular Dynamics Modeling of Thermodiffusion in Binary Mixtures: This chapter introduces an enhanced non-equilibrium MD approach, model predictions for a binary hydrocarbon mixture with experimental data. Multiple MD simulations were performed to calculate and compare Soret coefficient predictions of n-pentane/n-decane $\left(\mathrm{nC}_{5}-\mathrm{nC}_{10}\right)$, evaluated at $300.15 \mathrm{~K}$ and $0.1 \mathrm{MPa}$ for three different molar compositions. An augmented TraPPE-UA force field combined with the original HEX approach was first tested and compared to the numerical data obtained from the different force field models available in literature, which were based on the same BD-NEMD algorithm. Subsequently, the performance of an enhanced HEX algorithm was compared to the original HEX model, when coupled with the augmented TraPPE-UA.

Chapter 5 - Enhanced Molecular Dynamics Evaluation of Thermophobicity in Ternary Mixtures: This chapter further studies the feasibility of the previously proposed enhanced MD algorithm in ternary mixtures at five different compositions in the attempt to understand the physics behind the separation response in each mixture and the effect of cross-interactions between the molecules of different species influencing thermodiffusion behavior. The thermodiffusion factor or separation ratio of methane/n-butane/n-dodecane $\left(\mathrm{nC}_{1}-\mathrm{nC}_{4}-\mathrm{nC}_{12}\right)$ was assessed at $333.15 \mathrm{~K}$ and $35 \mathrm{MPa}$, and compared to the experimental data as well as to 3 other MD models from the literature.

\section{Chapter 6 - Molecular Dynamics evaluation of Alumina nanofluid thermal trans-} port performance: This chapter introduces an MD approach synergistically combined with augmented force field models to investigate the thermophysical properties and overall thermal performance of $\mathrm{Al}_{2} \mathrm{O}_{3}$-water nano fluid at different volumetric concentration and temperatures. In all the MD runs, alumina nano-composites were assumed to be suspended in an aqueous solution of an acidic $\mathrm{pH}$ level (in the rage of $3 \sim 3.75$ ) corresponding to the conditions at which one can prepare a stable well-dispersed nanofluids. To achieve the latter, the force fields were revamped by manipulating the solid-liquid interfacial atomic structure and electrostatic states in order to create positively charged alumina NPs, thereby bringing a closer solution of delineating methods for preparing and modulating the stability/aggregation of nanofluids.

Chapter 7 - Conclusions and Future Research Directions: This chapter provides 
a summary of the work completed in this thesis and gives suggestions for future work based on the contributions herein. 


\section{Chapter 2}

\section{Applications Background}

As there are many cases in which measurements and theoretical predictions are challenging, contradicting or impossible, MD simulations recreate a wide range of engineering problems to justify the discrepancies and fill the gap left by experimental discoveries and theoretical formulations.

In this thesis work, we attempt to illustrate specific aspects and validate the usefulness of the MD approach dealing with multicomponent thermofluid. This preliminary chapter introduces a brief background of the two engineering areas of interest to further contextualize and motivate the necessity of implementing enhanced MD techniques, starting with 1) thermodiffusion in hydrocarbon mixtures, and followed by 2) thermal transport of nanofluids for energy efficiency applications.

\subsection{Thermodiffusion in Petroleum}

When a temperature gradient is applied to a mixture, it produces not only a transport of energy but also a transport of mass. This non-equilibrium diffusive effect (the establishment of a concentration gradient under the influence of a temperature gradient) is called thermodiffusion or Soret effect [7]. Different constituents may drift to the cold or to the warm areas, and mixtures may display a relative accumulation in any of the two areas. Such a physical process is observed in a plethora of natural and technological applications including

petroleum, biology, environmental engineering as well as material science [7]. The separation 
appears in a wide variety of systems of different chemical nature, ranging from molecular liquids, gases and even solids, such as polymer solutions, biological fluids, oil reservoirs, charged electrolytes and colloidal dispersions, gas bubbles in liquids or aerosols. Evidently, most systems of scientific interest contain significantly more than two constituents [8].

The quantification of this effect still lacks a clear theory and explanation. In fact, a general principle capable of predicting thermodiffusion, from non-associating to associating mixtures, is still unclear as the response of species to a temperature gradient strongly depends on the chemical structure, interfaces and the ensuing cross-interactions mode. It has proven almost impossible to formulate a unified model that can adequately capture all aspects and atomic morphology influencing the separation of species [9].

During the last decade, the advances in computation and the accessibility of atomistic techniques such as MD have enabled the discovery of the molecular contributions on thermodiffusion in real physical systems: simulations have provided a way to mimic the nonequilibrium conditions and observe the unusual effects controlling the orientation of the fluid species [10, 11].

Our work was instigated by the extensive investigations and debates around quantifying thermodiffusion in hydrocarbons mixtures. The majority of oil and gas industrial interests focus on exploring and extracting petroleum fluids from geological formations and wells located in the deep crust [12]. Crude oil reservoirs mainly consist of multicomponent mixtures of varying structural shape and composition under the effect of gravity, chemical reactions and thermal gradients, with the Soret effect playing a decisive role in the establishment of their initial state. The empirical data of physical and transport properties of real reservoir fluids are relatively scarce: experimental techniques are expensive, difficult to perform and fail to cover the full range of working conditions. A comprehensive review of the available experimental studies of asymmetric ternary and multicomponent hydrocarbon mixtures is given in [12 15]. Modeling these mixtures remains a challenging task, due to the asymmetry in composition of such complex systems and the nature of cross-interactions between unlike particles.

Definitely, there is a lack of MD attempts when it comes to evaluating the effect of 
asymmetry on the degree of heat and mass transfer in ternary mixtures [16]. Most of the MD findings are limited to binary liquid mixtures, consisting of two components with similar masses [14]. The necessity to gain better insight into the molecular origin in multicomponent mixtures and quantify the thermodiffusive effect on the species distribution carried us to construct an enhanced MD algorithm capable to appropriately reflect the thermophobicity concept in hydrocarbon reservoirs.

\subsection{Thermal Performance in Nanofluids}

In the last few decades, dispersions of nanofluids have received a great attention in a large variety of thermal engineering applications. A nanofluid is a mixture of nanometersized solid particles suspended in a conventional fluid, such as water, oil or ethylene glycol [17]. The NPs could be metal particles, such as $\mathrm{Al}, \mathrm{Cu}$, and $\mathrm{Ni}$; oxides, such as $\mathrm{Al}_{2} \mathrm{O}_{3}, \mathrm{TiO}_{2}, \mathrm{CuO}, \mathrm{SO}_{2}, \mathrm{Fe}_{2} \mathrm{O}_{3}$ and $\mathrm{Fe}_{3} \mathrm{O}_{4}$; and some other composite materials, such as AlN, $\mathrm{SiC}$, and graphene [17]. Unique properties and phenomena arise when adding nanoparticles (NPs) to base fluids. Engineering nano has been offering tremendous opportunities by pushing nanoscale heat transfer frontiers in thermal management, dispatchable renewables, energy storages, sustainable harvesting for cooling/heating purposes, as well as nanofabrication techniques. [18, 19].

Nevertheless, exploring paradigms at the few-nanometer scale requires unprecedented precision, not readily possible through experimental techniques or conventional theories [20, 21. In particular, a complete description of nanoscale thermal behavior remains a great challenge, yet a promising platform for discovery to enable new and improved functionalities.

Complex physical and chemical adsorption phenomena are expected at the NP surface. The possible ion migrations upon surface hydration modify the chemical nature (surface density, electronic structure, morphology, etc.) and ultimately the thermophysical and rheological properties of the nanofluid. Far from being anecdotal, the various interactions found at the solid-liquid interface, involving pair potential forces, electrical layer action and steric action, largely govern NPs morphology by changing their dispersion, shape and aspect ratio $[22,23]$. 
Research on nanofluids has been tempered by a lack of consensus and clarity to identify the real heat transfer mechanisms behind the enhancement in thermal technologies, such as: aggregation level, electric charge ( $\mathrm{pH}$ level), Brownian motion, interfacial nanolayer, nanoclusters size and configuration [6, 24].

The heat transfer performance can greatly improve from high surface area to volume ratio of the NPs, especially at very low volume fraction [23]. But due to the strong interactions between them, NPs always tend to aggregate or settle out of solution under the influence of gravity [25]. The formation of clusters is a common occurrence during the preparation and processing of NPs, which results in not only sedimentation and clogging but also deterioration of the nano-colloidal characteristics, such as thermal conductivity, viscosity and specific heat capacity [25]. Maintaining a static nanofluid with a homogeneous suspension is crucial to extend the shelf-life of the nanofluid, while preserving its unique properties and functionality. Several physical and chemical treatments have been suggested to obtain stable nanofluids, including 1) the addition of stabilizing agents or surfactants, 2) pH regulation to modify particles electrostatics and solution ionic strength and 3) the application of powerful forces on nanoclusters using ultrasonic waves [25].

Adding nanomaterials to conventional fluid could lead to superior heat transfer performance; however, combining and optimizing the different thermophysical properties of nanofluids lead to performance tradeoffs and requires further investigations [22, 26, 27]. For example, most studies deal with thermal conductivity enhancement, and few examine viscosity; when they do, the data is rarely coupled. The dynamic viscosity is very sensitive to NP concentration or agglomeration state: the addition of NPs may favor the thermal conductivity, but is detrimental to the fluidity, due to unfavorable clustering and increased interparticles collisions [27, 28]. Therefore, it is important to give viscosity the same attention, due to its critical effect on the overall performance of thermal energy plants. In other words, an all-encompassing measurement of both thermal conductivity and viscosity of the nanofluid is essential to bring response elements on the actual enhancement of the heat transfer coefficient.

Despite many interesting phenomena described and understood, there are still several 
important issues that need to be solved for practical application. The winning composition of nanofluids meeting all engineering requirements (high heat transfer coefficients, long-term stability and low viscosity) has not been formulated yet, because of the complexity and multi-variability of nanofluid systems [26, 28]. 


\section{Chapter 3}

\section{Molecular Dynamics: Methodology, Applications, and Perspectives}

In this chapter, we proceed with an introduction to MD simulation, followed by a more detailed exposition of the methodology used to simulate the systems of interest pertinent to the present thesis work. More specialized preliminary information pertaining to the content of an individual chapter can also be found in this chapter.

\subsection{Foundation}

Today, computer simulations are an essential tool in scientific research. They serve as a complement to experiments, enabling us to understand and further develop systems that are not experimentally feasible in laboratory. In some cases, experiments are impossible to conduct: too dangerous, too expensive or incapable of detecting microscopic comportment's [11. Theories based on scarce experimental data carry these shortcomings in the form of approximations, assumptions and simplifications. Therefore, one can turn to molecular simulations to circumvent the pitfalls that exist in experiments and theories.

Molecular modeling acts as an intermediate between the classical models and laboratory measurements, being considerably more precise than theory and significantly less expensive than experiments. It does not substitute experimental measurement, but rather complements them and validates the theoretical models. Molecular models are particularly useful 
for constructing complicated molecular systems, visualizing 3D structures and understanding the relationship between structure, dynamics and function. The typical scale of molecular simulations (ranging from less than 1 nanometer to a micrometer) helps address chemical reactions, equilibrium properties and transport phenomena [29]. Depending on the type of problem addressed, atomic structure can be defined at very high resolution, using QuantumMechanics (QM), with every single electron modelled, or at coarser levels with Molecular Dynamics (MD), where the smallest particles represented are atoms or parts of molecules. The former comes at the expense of severe computational cost [30]. Due to the multitudinous interactions between electrons of atoms and molecules, QM struggles to deal with macromolecules [31]. Put differently, it is simply too expensive to reproduce the collective dynamic behavior of larger systems, like the ones adopted in this work.

Reliable MD simulations can be performed on a variety of systems, thanks to the development of numerous ingenious algorithms which save computer time, while nevertheless exploring all possible configurations. In the light of the applications in this thesis, MD serves as the most suitable method. In the following sections, we provide a background discussion of the main theoretical ingredients and methodology of the MD approach. We first introduce the formulations of classical MD algorithms and the Force Field models, followed by a description of the Linear Response Theory to derive the thermal transport properties. Finally, we represent the fundamental features of Equilibrium MD (EMD) and Non-Equilibrium MD (NEMD) approaches adopted for the calculation of the system properties with regard to the applications of this thesis project.

It must be noted that the description of any additional information required to simulate the systems of interest can be found in the chapter of the corresponding application.

\section{Mathematical Formulation}

In MD, the dynamics of a system consisting of an ensemble of atomic particles is studied by numerically integrating Newton's laws of motion (see Eq. (3.1) for each particle, using prescribed interatomic potentials [11]. 


$$
\begin{aligned}
& \boldsymbol{F}_{\boldsymbol{i}}=m_{i} \frac{d^{2} \boldsymbol{r}_{\boldsymbol{i}}(t)}{d t^{2}} \\
& \boldsymbol{F}_{i}=-\frac{\partial U\left(\boldsymbol{r}_{\boldsymbol{i}}, \ldots \boldsymbol{r}_{\boldsymbol{N}}\right)}{\partial \boldsymbol{r}_{\boldsymbol{i}}}
\end{aligned}
$$

Where $F_{i}$ is the force acting on atom $i(1,2, \ldots, N)$ with mass $m_{i}$ and $t$ denotes the time. MD simulation requires calculation of the gradient of the potential energy $U(r)$, which therefore must be a differentiable function of the atomic coordinates $r_{i}$.

The trajectories generated by time integration schemes correspond to a sequence of system microstates. Assuming ergodicity, these microstates can be sampled to obtain thermodynamic quantities and system properties at equilibrium as well as steady state [32]. Static equilibrium quantities can be obtained by averaging over the trajectory, which must be of sufficient length to form a representative ensemble of the state of the system. In addition, dynamic information can be extracted [32, 33]. The microcanonical averages can be computed according to Eq. (3.2) or (3.3) as shown below:

$$
\langle A\rangle=\lim _{\tau \rightarrow \infty} \frac{1}{\tau} \int_{0}^{\tau} A[x(t)] d t \equiv \bar{A}
$$

where $x(t)$ is a representative point of the phase space defined as $x(t)=\left(\mathbf{r}^{N}(t), \mathbf{p}^{N}(t)\right)$ where $\mathbf{r}$ and $\mathbf{p}$ denote the position and momentum vectors respectively. This formula can be discretized for MD simulations as follows:

$$
\langle A\rangle=\frac{1}{M} \sum_{n=0}^{M} A\left(x_{n \Delta t}\right)
$$

Another asset of MD simulation is that non-equilibrium properties can be efficiently studied by keeping the system in a steady non-equilibrium state, as discussed in the following sections. 


\section{Assumptions, Approximations and Limitations}

When using a particular MD model to predict the properties of a molecular system, one should be aware of the assumptions, simplifications, approximations and limitations that are implicit in the model. Many simulation factors such as the system size, processes timescale, the accuracy of the potential field as well as the precision of the integration schemes radically impact the validity of the MD results [11].

\subsection{Force Field Models}

The force field is one of the most crucial part of MD simulations. Cast in the form of a simple mathematical function, it must faithfully represent the interaction between atoms. It is used to describe the intramolecular and intermolecular potential energy of a collection of atoms, and the corresponding parameters that will determine the energy of a given configuration [30]. These functions and parameters have been derived from experimental results and quantum mechanical calculations of small model compounds. They are often refined by computer simulations to compare calculated condensed phase properties with experiment or reproduce benchmarked results of system properties [34].

However, approximations and simplifications lead to limitations in transferability and reliability of MD results. Simplifying assumptions in the force field model alter the results of MD predictions [11]. These inaccuracies call for more thorough potential fields, with minimal use of assumptions. Improvement of the quality and extension of the range of applicability of the potential energy functions is a continual concern in the area of MD [10. Though some

applications require more refined force fields than others, there should be a balance between the level of refinement and the computational efficiency.

\section{Mathematical Formulation}

As shown in Eq. (3.1b), the force acting on an atom $i$ is calculated as the negative gradient of a scalar potential energy function $U$ that depends on all atomic positions and, thereby, couples the motion of atoms. For systems of biomolecules, this potential energy function is 
usually divided in two parts [35]:

$$
U=U_{\text {bonded }}+U_{\text {non-bonded }}
$$

The bonded potential $U_{\text {bonded }}$ involve 2,3 , and 4-body interactions of covalently bonded

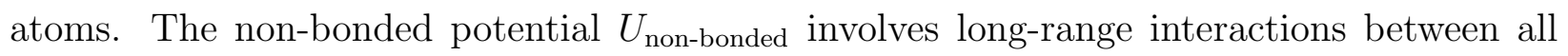
pairs of atoms (usually excluding pairs of atoms already involved in a bonded term).

\section{Bonded potential terms}

The bonded potential describes the stretching, bending, and torsional of the covalent bonds.

\section{- Bond stretching:}

The bond stretching term is a 2-body potential, generally assumed to be harmonic, that describes the vibrational motion between a pair of covalently bonded atoms:

$$
U_{b}(r)=\sum_{b o n d s} \frac{k_{b}}{2}\left(b-b_{0}\right)^{2}
$$

where $b$ is the distance between the two atoms. Two parameters characterize each bonded interaction: $b_{0}$ the average distance between them and a force constant $k_{b}$.

\section{- Angle bending:}

The angle bending terms describes the force originating from the deformation of the valence angles between three covalently bonded atoms (3-body interactions). The angle bending term is described using a harmonic potential:

$$
U_{\theta}(r)=\sum_{\text {angles }} \frac{k_{\theta}}{2}\left(\theta-\theta_{0}\right)^{2}
$$

where $\theta$ is the angle between three atoms. Two parameters characterize each angle in the system: the reference angle $\theta_{0}$ and a force constant $k_{\theta}$.

\section{- Torsional terms:}

The torsional terms are weaker than the bond stretching and angle bending terms. They describe the barriers to rotations existing between four bonded atoms (4-body interaction). 
There are two type of torsional terms: proper and improper dihedrals. Proper torsional potentials are described by a cosine function:

$$
U_{\delta}(r)=\sum_{\text {proper }} \frac{k_{\delta}}{2}[1+\cos (n \delta-\gamma)]
$$

where $\delta$ is the angle between the planes formed by the first and the last three of the four atoms. Three parameters characterize this interaction: $\gamma$ sets the minimum energy angle, $k_{\delta}$ is a force constant and $n$ is the periodicity.

The improper dihedral term is designed both to maintain chirality about a tetrahedral heavy atom and to maintain planarity about certain atoms. The potential is described by a harmonic function:

$$
U_{\omega}(r)=\sum_{\text {improper }} \frac{k_{\omega}}{2}\left(\omega-\omega_{0}\right)^{2}
$$

where $\omega$ is the angle between the plane formed by the central atom and two peripheral atoms and the plane formed by the peripheral atoms.

\section{Non-bonded potential terms}

The non-bonded potential describes the Van der Waals forces and the electrostatic interactions between the atoms.

\section{- Van der Waals interactions}

The Van der Waals force acts on atoms in close proximity. It is strongly repulsive at short range and weakly attractive at medium range. The interaction is described by a Lennard-Jones (LJ) potential:

$$
U_{V d W}\left(r_{i j}\right)=4 \epsilon_{i j}\left[\left(\frac{\sigma_{i j}}{r_{i j}}\right)^{12}-\left(\frac{\sigma_{i j}}{r_{i j}}\right)^{6}\right]
$$

where $r_{i j}$ is the distance between atoms $\mathrm{i}$ and $\mathrm{j}$. It is parameterized by $\sigma$ : the collision parameter (the separation for which the energy is zero) and $\epsilon$ the depth of the potential well. The LJ potential approaches 0 rapidly as $r$ increases, so it is usually truncated (smoothly shifted) to 0 past a cutoff radius.

\section{- Electrostatic interactions}


Finally, the long distance electrostatic interaction between atoms $\mathrm{i}$ and $\mathrm{j}$ with charges $q_{i}$ and $q_{j}$ at a distance $r_{i j}$ is described by the Coulomb's law:

$$
U_{e}(r)=\sum_{i=1}^{N} \sum_{i \neq j} \frac{q_{i} q_{j}}{r_{i j}}
$$

The final equation for the potential energy describing the force field can be expressed as a summation of all the above energy modes:

$$
\begin{aligned}
& U(r)=\sum_{\text {bonds }} \frac{k_{b}}{2}\left(b-b_{0}\right)^{2}+\sum_{\text {angles }} \frac{k_{\theta}}{2}\left(\theta-\theta_{0}\right)^{2}+ \\
& \left.+\sum_{\substack{\text { proper } \\
\text { dihedrals }}} \frac{k_{\delta}}{2}\left[1+\cos \left(n \delta-\delta^{*}\right)\right]+\sum_{\begin{array}{c}
\text { improper } \\
\text { dihedrals }
\end{array}} \frac{k_{\omega}}{2}\left(\omega-\omega_{0}\right)^{2}+\right\}^{\begin{array}{c}
\text { Bonded } \\
\text { interactions }
\end{array}} \\
& +\sum_{i=1}^{N} \sum_{i \neq j} 4 \varepsilon_{i j}\left[\left(\frac{\sigma_{i j}}{r_{i j}}\right)^{12}-\left(\frac{\sigma_{i j}}{r_{i j}}\right)^{6}\right]+\sum_{i=1}^{N} \sum_{i \neq j} \frac{q_{i} q_{j}}{r_{i j}} \Longrightarrow \quad \begin{array}{c}
\text { Non-bonded } \\
\text { interactions }
\end{array}
\end{aligned}
$$

\subsection{Linear Response Theory: Heat and Mass Trans- port}

Generally, the linear phenomenological theory postulates that the fluxes are linear homogeneous functions of the corresponding gradients. This linearity is expressed as [33, 36]:

$$
\boldsymbol{J}_{\alpha}=\sum_{\beta} L_{\alpha \beta} X_{\beta}
$$

where $\boldsymbol{J}_{\alpha}$ is the flux of the ith physical quantity (component), $X_{\beta}$ the $\beta^{\text {th }}$ thermodynamic force and $L_{\alpha \beta}$ the corresponding phenomenological coefficients. According to the Onsagers reciprocity hypothesis, $L_{\alpha \beta}=L_{\beta \alpha}$, which assumes microscopic reversibility or local equilibrium. The fluxes and gradients (or thermodynamic forces) are connected to the second law 
of thermodynamics through the entropy production $\sigma$ given by

$$
\sigma_{s}=\sum_{\alpha} \boldsymbol{J}_{\alpha} \boldsymbol{X}_{\alpha}
$$

In the absence of external forces and chemical reactions and assuming no viscous flow, the entropy production

$$
\sigma_{s}=\boldsymbol{J}_{U} \cdot \frac{\nabla T}{T^{2}}-\sum_{k=1}^{n-1} \boldsymbol{J}_{k} \cdot \nabla\left(\frac{\mu_{k}-\mu_{n}}{T}\right)
$$

where $\boldsymbol{J}_{U}$ is the internal energy flux, $\boldsymbol{J}_{k}$ is the mass flux of component $k, T$ is the temperature, $\mu_{k}$ is the partial specific Gibbs energy of component k. The summation in the second term of (3.14) is over all species except one, since one mass flux $\boldsymbol{J}_{n}$ is eliminated by making use of $\sum_{i=1}^{n} \boldsymbol{J}_{i}=0$ since the variables within one set should independent [37].

The mass flux of species $k$ in the barycentric frame of reference of the mixture is given by:

$$
\boldsymbol{J}_{k}=\frac{1}{V} \sum_{j=1}^{N_{k}}\left(\boldsymbol{v}_{j}-\boldsymbol{v}\right)
$$

where $\boldsymbol{v}$ denotes the barycentric velocity and $\boldsymbol{v}_{j}$ is the velocity of the $j^{\text {th }}$ particle of type $k$. Irwing and Kirkwood [36] have derived an instantaneous and microscopic expression for the internal energy flux $\boldsymbol{J}_{U}$. In this work, we use the definition given by [38]:

$$
\boldsymbol{J}_{q}=\boldsymbol{J}_{U}-\sum_{k=1}^{n-1}\left(h_{k}-h_{n}\right) \boldsymbol{J}_{k}
$$

where $\boldsymbol{J}_{U}$ is the internal energy flux and $\boldsymbol{J}_{q}$ is the 'reduced' heat flux that is responsible for conduction in a multi-component system. $h_{i}$ is the partial specific enthalpy of species i. For an ideal mixture, $h_{k}$ is independent of the composition and equal to the specific enthalpy of pure component $k$. In this work, we shall assume hydrocarbon liquid mixtures as an isotope quasi-ideal mixture with molecular masses $m_{k}$, which leads to $h_{k}=m_{n} h_{n} / m_{k}$. Therefore Eq. 3.15 can be written as:

$$
\boldsymbol{J}_{q}=\boldsymbol{J}_{U}-h_{n} \sum_{k=1}^{n-1}\left(\frac{m_{n}}{m_{k}}-1\right) \boldsymbol{J}_{k}
$$


Onsager reciprocal relations [39] describe the coupling of heat and mass transfer in a multicomponent mixture, such that the macroscopic flux is expressed as a linear combination of the temperature and concentration gradient forces. These relations are expressed in the following way:

$$
\begin{aligned}
& \boldsymbol{J}_{q}=L_{q q} \boldsymbol{X}_{q}+\sum_{j=1}^{n-1} L_{q j} X_{j} \\
& \boldsymbol{J}_{k}=L_{k q} \boldsymbol{X}_{q}+\sum_{k=1}^{n-1} L_{i k} X_{k}
\end{aligned}
$$

with $\quad \boldsymbol{X}_{q}=-\frac{\nabla T}{T^{2}}$ and $\quad \boldsymbol{X}_{k}=-\frac{\nabla\left(\mu_{k}-\mu_{n}\right)_{T}}{T}$.

For a simple binary system with 2 components $(\alpha, \beta)$, the phenomenological relationships reduces to

$$
\begin{aligned}
& \boldsymbol{J}_{q}=-L_{q q} \frac{\nabla T}{T^{2}}-L_{q 1} \frac{1}{T} \nabla\left(\mu_{k}-\mu_{n}\right)_{T}, \\
& \boldsymbol{J}_{\alpha}=-L_{1 q} \frac{\nabla T}{T^{2}}-L_{11} \frac{1}{T} \nabla\left(\mu_{k}-\mu_{n}\right)_{T}
\end{aligned}
$$

Using the Gibbs-Duhem identity in terms of the concentration $w \sum_{k=\alpha}^{\beta} w_{k}\left(\nabla \mu_{k}\right)_{T, P}=0$ and the relation $\left(\nabla \mu_{k}\right)_{T, P}=\left(\frac{\partial \mu_{\alpha}}{\partial w_{\alpha}}\right)_{T, P} \nabla w_{\alpha}$ Eq.3.18a can be expressed as:

$$
\begin{aligned}
& \boldsymbol{J}_{q}=-L_{q q} \frac{\nabla T}{T^{2}}-L_{q 1} \frac{1}{T w_{\beta}}\left(\frac{\partial \mu_{\alpha}}{\partial x_{\alpha}}\right)_{T, P} \nabla x_{\alpha}, \\
& \boldsymbol{J}_{\alpha}=-L_{1 q} \frac{\nabla T}{T^{2}}-L_{11} \frac{1}{T w_{\beta}}\left(\frac{\partial \mu_{\alpha}}{\partial w_{\alpha}}\right)_{T, P} \nabla w_{\alpha},
\end{aligned}
$$


Alternatively, the set of flux relationships can be written as:

$$
\begin{aligned}
& \boldsymbol{J}_{q}=-k \nabla T-\rho\left(\frac{\partial \mu_{\alpha}}{\partial w_{\alpha}}\right)_{T, P} T D_{T} \nabla w_{\alpha}, \\
& \boldsymbol{J}_{\alpha}=-\rho w_{\alpha} w_{\beta} D_{T} \nabla T-\rho D_{12} \nabla w_{\alpha},
\end{aligned}
$$

At the stationary state when $\boldsymbol{J}_{\alpha}=0$, the thermodiffusion factor or Soret coefficient can be obtained from Eq.3.20(b), respectively:

$$
\begin{gathered}
\alpha_{T}=T \frac{D_{T}}{D_{12}}=-\frac{T}{w_{1} w_{2}}\left(\frac{\nabla w_{1}}{\nabla T}\right)_{\boldsymbol{J}_{1}=0} \\
S_{T}=\frac{D_{T}}{D_{12}}=-\frac{1}{w_{1} w_{2}}\left(\frac{\nabla w_{1}}{\nabla T}\right)_{\boldsymbol{J}_{1}=0}
\end{gathered}
$$

Further details of linear response theory a basis for are given in [36, 40]. The energy flux in a control volume (CV) is given in MD by [38]:

$$
\boldsymbol{J}_{U}=\frac{1}{V} \sum_{i \in C V}\left(\left[\frac{1}{2} m_{i}\left(v_{i}-v\right)^{2}+\delta_{i}\right]\left(v_{i}-v\right)-\frac{1}{2} \sum_{j=1}^{N}\left[\left(v_{i}-v\right) . \boldsymbol{F}_{i j}\right] \boldsymbol{r}_{i j}\right)
$$

where $V$ is the size of the control volume, $m_{i}$ and $v_{i}$ the mass and velocity, respectively, of particle $\mathbf{i}, \mathbf{v}$ is the barycentric velocity of the system, $\delta_{i}$ is the potential energy of particle $i$ in the field of all other particle, $\boldsymbol{F}_{i j}$ is the force action on $i$ due to $j$, and $\boldsymbol{r}_{i j}$ is the vector from the position of $i$ to the position of $j$. This a local, instantaneous analogue to the macroscopic internal energy flux. The full expansion for a two component system results in [32]. The evaluation of partial enthalpies requires simplifying assumptions. The mean enthalpy can be computed as the sum of the mean kinetic and potential energies, and the virial [32]. In this work, the computation of the partial enthalpy of species $\alpha$, specifically conducted in EDM simulations for the nanofluids systems, was described as the below:

$$
h_{\alpha}=\frac{1}{N_{\alpha}} \sum_{i=1}^{N_{\alpha}}\left[K_{i}+P_{i}+\frac{1}{3}\left(m_{i} v_{i}^{2}+\frac{1}{2} \sum_{j=1}^{N} \boldsymbol{r}_{i j} \cdot \boldsymbol{F}_{i j}\right)\right]
$$


Generally speaking, partial enthalpies are thermodynamic functions not readily accessible from molecular simulations [36]. Their decomposition into kinetic, potential and virial is true for isotopic or equi-potential mixtures, and in less restrictive ideal mixtures. Note that beside the reliability of the potential field model, the choice of the microscopic heat flux formulation also has a substantial impact on the calculation of the transport properties. A commonly accepted definition follows from the second law of thermodynamics and for an $\mathrm{n}$ component system, it is given by 32 ]

\subsection{Algorithms for Transport Coefficients Calcu- lation}

The computation of thermal transport properties via MD simulations has evolved into two main approaches: Equilibrium (EMD) and Non-Equilibrium (NEMD). EMD involves sampling the fluctuation of various fluxes spontaneously generated in the system to predict thermophysical properties on the system such as density, viscosity, diffusion coefficients and others. NEMD involves applying a temperature gradient to a system via imposed boundary conditions and then directly measuring the systems response.

\subsubsection{Equilibrium Molecular Dynamics (EMD)}

A system is said to be in equilibrium when its intensive state variables such as density and temperature are invariant with time and all the gradients of the variables are absent. So under equilibrium conditions there are no macroscopic flow of mass, momentum and energy.

According to LRT, the transport coefficients can be expressed as time correlation function of appropriate autocorrelation functions. In an equilibrium state, spontaneous fluctuations occur all the time, and time correlations are spatio-temporal functions that describe these fluctuations [37]. The connection between these functions and transport properties comes from the celebrated Onsager's regression hypothesis [37]. Generally, the time correlation functions are calculated by averaging the appropriate dynamic variables over time using EMD simulations and invoking the ergodic hypothesis of the equivalence of time and ensemble averaging [36]. Hence, the advantage of EMD begins by making the dynamical details of the temporal behavior of the heat flux correlation functions accessible for analysis. This 
method is also known as the Green-Kubo (GK) formalism [36]. A detailed description of GK formulation can be found in [32].

\section{Application in Nanofluids}

In this work, EMD approach was selected over NEMD to calculate the transport properties of nanofluids, namely thermal conductivity and viscosity. Since nanofluids are characterized with both spatial inhomogeneity and non-ideality, an extreme temperature gradient can adversely affect the dynamics at the solid-liquid interface, especially since cross-interactions between the base fluid (water) and the NPs (aluminum oxides) are significant. Therefore, the system to deviate from the linear response regime. Finally, the computational burden in EMD is greatly reduced compared to NEMD.

Note that stationary equilibrium period will depend on the relaxation time of the selected property. During a simulation, a number of quantities are monitored to ensure the stability of the simulation. The results are analyzed by taking time averages or averages over simulations with different initial conditions of the quantities of interest. Fluctuations and correlation functions are also calculated to interpret the mobility and dynamic behavior of the system [11, 32].

\subsubsection{Non-Equilibrium Molecular Dynamics (NEMD)}

Also based on LRT, NEMD has evolved into a powerful technique for predicting many physical and transport properties of many-body systems, principally driven out of equilibrium by an external thermodynamic force [38]. As with EMD simulations, the properties are computed as averages over time in a stationary state. Nevertheless, the main downside of NEMD is that it requires large perturbation strengths at the macroscale to generate detectable microscale responses larger than statistical noise effects of the system.

NEMD formulation is based on the linear irreversible thermodynamics, which assumes local equilibrium state [36]. However, the large thermal gradients (up to $10^{10}-10^{11} \mathrm{k} / \mathrm{m}$ ) encountered in typical NEMD simulations, one might expect a divergence from the microscopic reversibility or local equilibrium distribution leading to large uncertainties of the calculations [41]. It is then essential to check that the criteria of local equilibrium conditions are satisfied for NEMD simulations, especially in asymmetric systems. The validity of local equilibrium theory entails that local volume elements are small enough so that the thermodynamic prop- 
erties vary little over each layer, but large enough so that each control volume is treated as a macroscopic thermodynamic subsystem with small local fluctuations of its properties with respect to the gradients (i.e. temperature and particles number fluctuations) [42].

The nature of the coupling between the external field and the system differs between NEMD algorithms. The most suitable for a particular application is determined by its ability to mimic the underlying physics.

\section{Application in Hydrocarbon Soret effect}

The most representative NEMD algorithms applied in thermodiffusion and heat conduction studies are: synthetic NEMD (S-NEMD) also called homogeneous NEMD where the external thermodynamic force is built into the equations of motion for the system, and BoundaryDriven (BD) or direct NEMD where the external force stems from perturbations of the systems boundaries [43]. The main drawback of this method is that it requires the knowledge of additional thermodynamic quantities for the quantification of Soret coefficients[11]. One can avoid the calculation of these quantities by using BD-NEMD approach, which directly mimics the thermodiffusion procedure of the most pertinent experimental techniques [38. The method is based on the following modification of the system boundaries: the simulation box is partitioned into equal slabs along one direction with periodic conditions; heat is added to one region and removed from the other in order to create two reservoirs, with one acting as a heat sink and the other as a source. As a consequence, an energy flux is induced across the system, leading to well-developed temperature and concentration gradients at steady state [38].

One strategy to impose a fixed heat flux in the MD simulations involves swapping kinetic energy between the two regions of the simulation box. The heat exchange (HEX), developed by Hafskjold et al. [38], and the momentum exchange (PEX), developed by Mller-Plathe [43], are the most well-known algorithms based upon this concept. In the HEX method, a specific amount of heat flux is periodically subtracted from one subdomain (creating a cold reservoir) and added to another (generating a hot reservoir) by adjusting the non-translational kinetic energy through velocity rescaling while preserving the individual center of mass velocities of each subdomain [38]. In the PEX algorithm, the thermal flux is carried out through a 
momentum exchange between the particle with the highest kinetic energy in the cold region and the particle having the lowest kinetic energy in the hot region, continuously. In both cases, a stationary state is reached, and the system properties can be computed [43].

In particular, the HEX method prevails in the field of thermodiffusion studies where theories and experimental techniques fail to understand the underlying cross-coupling effect of transport mechanisms and quantify the Soret coefficients of the system components [44]. The method does not necessarily require an exact knowledge of the microscopic heat flux, which makes them highly attractive to assess the nature of heat and mass transport in multicomponent systems.

In summary, an MD technique based either on empirical force fields or quantum-based ab-initio molecular forces is a powerful tool, both as a means of probing equilibrium and non-equilibrium phenomena and for obtaining dynamical properties of systems [30].

The contents of the next chapters will highlight a number of challenging problems and limitations, which are well beyond the reach of simulation methods because of the size of the configurational space involved, the time scale of the process or the complexity of the system components. 


\section{Chapter 4}

\section{Enhanced Molecular Dynamics Modeling of Thermodiffusion in Binary Mixtures}

This chapter has been reproduced, nearly exactly, from our previously published work to refer directly to Chapter 4 of this thesis. The publication originally appears as in [8]: S. Antoun, M. Z. Saghir, and S. Srinivasan, "An improved molecular dynamics algorithm to study thermodiffusion in binary hydrocarbon mixtures."

\subsection{Introduction}

Ludwig - Soret effect, also known as thermodiffusion, is the process of induced mass transport of chemical species in liquid or gaseous convection-free mixtures in response to a thermal gradient [13]. The strength and direction of the separation process are quantified by the Soret coefficient or thermal diffusion factor. The latter is measured at stationary state when the net mass flux of the mixture vanishes and the temperature and composition gradients are well developed in the linear regime. For the simple case of a binary mixture, is defined as:

$$
S_{T}=-\frac{D_{T}}{D_{12}}=\frac{\nabla w_{1}}{w_{1}\left(1-w_{1}\right) \nabla T}
$$


Thermal diffusion plays an important role in several natural and industrial practices. For instance, it plays a key factor in component segregation in petroleum reservoirs [12], formation of the salt concentration in different layers of oceans [45] as well as convection in stars. From the industrial point of view, the Soret effect is used in isotope separation of liquids and gaseous mixtures [46], thermal osmosis [47], mass transport across biological membranes [48] and thermophoretic trapping of DNA [49]. In the past 20 years, different type of experimental methods (optical and non-optical) have been developed for thermodiffusion investigations.However, accurate measurements of are very difficult to perform due to the complexity of the process: the magnitude of the effect is very small which renders its measurement challenging under laboratory conditions, in addition to possible distortion due to mechanical disturbances. A detailed review of the experimental investigations and techniques of thermodiffusion is found in [50, 51]. Despite recent progress, experimental data are still scarce and lack consensus when using different techniques. On the experimental front, errors can creep in due to the several external factors including natural gravity, mechanical vibrations, and the handling of the mixtures constituents during the post-processing of the experiment [52].

On the other hand, a lot of theoretical work has been advanced in this field in order to predict the thermodiffusion coefficient of binary and ternary mixtures. Most of these models are based on non-equilibrium thermodynamic theory for $S_{T}$ estimation. Eslamian and Saghir [53] presented a comprehensive review of these theoretical approaches. Despite the development of several theories, there are still discrepancies and discontinuity among the models in producing accurate results of thermal diffusion. The reason for the disagreement is the complexity of understanding the microscopic contributions of the phenomena.

Among other computational techniques, MD has served as a highly promising method and a low-cost alternative for experiments to estimate thermodynamic and transport properties, including thermal diffusion coefficients [1, 39, 43, 44, 54,58. Many approaches based on nonequilibrium statistical mechanics have been developed to compute the Soret coefficient in binary and ternary mixtures [1, 43, 44, 54]. Mozaffari et al. [57] presented a detailed comprehensive review and comparison between the different MD methods based on the data from the literature (theoretical as well as experimental). They investigated the behavior of two well-known boundary-driven non-equilibrium molecular dynamics (BD-NEMD) approaches, 
namely, Reverse Non-Equilibrium Molecular Dynamics (RNEMD) and heat exchange (HEX) algorithm. They also introduced a Modified HEX (MHEX) model that showed to be more accurate and computationally efficient to simulate the mass and heat transfer mechanism. Perronace et al. [44] did an extensive study of Soret coefficients on n-pentanen-decane mixtures for three different weight fractions using EMD, synthetic (S-NEMD), and BD-NEMD simulations. Their results showed good agreement with the experimental results. Zhang and Muller-Plathe [43] implemented the RNEMD method to investigate the thermal diffusion of benzene-cyclohexane mixtures using an all-atom (AA) model with partial charges. They studied the influence of different parameters such as the cutoff distance and the perturbation strength. They also evaluated the composition and temperature dependence on the Soret coefficient. Galliero et al. [59] conducted BD-NEMD simulations to examine thermodiffusion coefficients of methane $\left(\mathrm{nC}_{1}\right)$, butane $\left(\mathrm{nC}_{4}\right)$, and dodecane $\left(\mathrm{nC}_{12}\right)$ ternary mixtures at 333.15 $\mathrm{K}$ and $35 \mathrm{MPa}$ for 5 different molar compositions using different fluid models: Lennard-Jones (LJ) and Lennard-Jones Chain (LJC) fluid models. Later, Artola and Rousseau [54] performed extensive MD simulations on ternary isotopic mixtures using the LJ sphere model. They computed the Soret coefficients of 440 different systems and investigated the effect of 63 different compositions and 7 sets of mass ratio using the HEX approach.

As pointed out by Wirnsberger et al. [58], several studies have reported round-off and truncation errors related with approximate integration schemes and limited computational precision. These errors have a significant impact on time averages of transport coefficients, mainly when the algorithms convergence and stability time scales are long. They proved that the numerical implementation of the HEX method leads to a considerable drift in the total system energy over the simulation run time. For long simulations, this energy loss becomes restrictive, limiting the accessible simulation time scales to a few nanoseconds. Wirnsberger et al. [58] proposed a new algorithm for boundary-driven (BD-NEMD) simulations called enhanced HEX (eHEX) that entails an extension of the HEX algorithm. The method proposes an additional coordinate integration step to minimize the truncation error coming from the operator splitting. The eHEX MD simulations exhibited an excellent conservation of energy, even for large time steps when compared to the HEX method.

The goal of the work of Mozaffari et al. [57] was to minimize the computational time, while maintaining reasonable accuracy. Nevertheless, to guarantee that the performance 
observed in NEMD simulations is representative of real dynamical systems, the selection of an accurate force field is essential.A great deal of work has been conducted over the past decade in developing potentials suitable for reproducing equilibrium transport properties and a benchmark based on their density and viscosity prediction of n-alkane under ambient and HighTemperature High Pressure (HTHP) conditions [29, 35, 59 68]. Two main approaches are commonly adopted when modeling dispersion and repulsive interactions between polyatomic molecules: (i) all-atom (AA) models; (ii) united-atom (UA) models. The benefit of AA models is that they describe better the details of the molecular configuration and structure directly associated with the dynamic properties [35, 62]. However, they are computationally very expensive when simulating large systems with complex geometries. Whereas, the UA potentials treat a specific group of atoms, such as $\mathrm{CH}_{2}$ and $\mathrm{CH}_{3}$, as simple Lennard-Jones sites with single force centers [60]. This approximation has been widely adopted in recognition of the fact that more efficient computations of various properties are gained in reducing the total number of interaction sites especially in large systems that require long simulations.

Several UA models have been proposed to predict the thermodynamic properties of nalkanes with good performance [35, 64], Significant issues with these force fields were highlighted for the simulation of linear alkanes for different chain size and under specific conditions. More specifically, all of the UA force fields under-predicted the viscosity and friction of long-chain linear molecules, and the AA force fields ensued a raise in the melting point for nalkane, leading to anomalous density and viscosity values, and subsequently a discrepancy in diffusion coefficient predictions [35, 64]. These problems are expected to affect the thermodiffusion process in confined NEMD simulations. Nevertheless, a lot of research studies have recently focused on resolving this weakness by modifying model parameters to have a better representation of the experimental data for transport properties of n-alkanes. For instance, Ungerer et al. [? ] suggested improving the anisotropic united atom (AUA4) model [66] by increasing the energetic barriers of the torsion intramolecular potential and enhancing the resulting internal dynamics of n-alkanes expressed in transport properties. Later, Galliero [59] showed that employing an additional rigidity fourth molecular parameter to the fully flexible Lennard-Jones Chain (LJC) model can serve as a mean to circumvent the problem and get a better description of simultaneously thermodynamic and transport properties of not too short n-alkane chains. However, adding the influence of a rigidity parameter requires 
the modification of the existing equation of states and transport properties correlations, which can be time consuming for MD simulations.

The transferable potentials for phase equilibria-united atom (TraPPE-UA) force field, developed by Martin and Siepmann [60], have been used extensively in simulations of complex chemical systems, and it appears superior compared to other potential fields in its application to model bulk and interfaces transport properties in a wide range of states for both pure and mixtures of linear n-alkanes [64, 67]. Although the model underestimates the viscosity relative to experimental values, Dysthe et al. 64] proved that TraPPE-UA properly captures the dependence between the molecular structure, diffusion coefficient, and viscosity of hydrocarbons by performing multiple MD simulations. According to Ewen et al. [35], L-OPLSAA (long chain optimized potentials for liquid simulation) showed a better prediction of long-chain n-alkane properties; however, it is computationally more demanding when simulating large systems and has not proven to be more accurate for transport properties of short linear alkanes than TraPPE-UA. In the original TraPPE-UA, all pseudo atoms along the alkane chain are connected with bonds of fixed length. However, recent calculations of diffusion coefficients using a variation of the TraPPE-UA model, with flexible bonds, were performed on pseudo-atoms in n-alkanes mixtures [67]. In their work, Makrodimitri et al. [67] showed that the TraPPE-UA force field augmented with a harmonic bond stretching potential agree well with the experimental data for small molecules in heavy hydrocarbons and can be implemented to predict accurately system densities and diffusion coefficients in mixtures. From the above literature survey, it is clear that TraPPE-UA is potentially a very good candidate for modeling interactions between n-alkanes with the least computational burden.

Keeping the advantages of the various approaches in mind, in this work, we propose to implement the eHEX NEMD algorithm along with the augmented TraPPE-UA to study thermodiffusion in a real system of hydrocarbons. The feasibility of this approach is demonstrated by calculating the thermodiffusion factor of the binary mixture $\mathrm{nC}_{5}-\mathrm{nC}_{10}$ for three different compositions at $300.15 \mathrm{~K}$ and $0.1 \mathrm{MPa}$ and by comparing the results with experimental and other MD numerical data available in the literature. In doing so, this paper is organized as follows: In section 4.2, a brief description of the theoretical concepts of thermodiffusion MD and potential field model formalisms is provided. In section 4.3, the 
methodology details are outlined for both EMD and NEMD simulations. Then, an analysis of the validity of computational results pertaining transport properties and the Soret coefficient of liquid mixtures is discussed in section 4.4 Finally, the summary of our investigation and the pertinent conclusions are drawn in section 4.5 .

\subsection{Theoretical Formulation}

The basics of the MD method rely on classical mechanics, determining the trajectory in the phase space of the system and taking time averages of the observables of interest along this trajectory. At each step of the MD simulation, the microscopic coordinates and momentum of all the particles were calculated according to equations of classical mechanics where forces are computed from the force field. In the ensuing paragraphs, the methodology and computational aspects adopted in this work are briefly described.

\subsubsection{The Enhanced Heat Exchange (eHEX) Algorithm}

The enhanced heat exchange (eHEX) algorithm proposed by Wirnsberger et al. [58] is an extension of the HEX method derived with a higher-order truncation error of the time integration algorithm. It consists of applying a constant heat flux onto the system by adding heat $\Delta Q_{r}$ into the hot region $\Gamma_{1}$ and subtracting the same amount of energy from the cold region $\Gamma_{2}$ at each time step (see Figure 4.1). As a consequence, the net energy flux of the system is zero and a stationary state is reached where a temperature gradient and internal energy fluxes are established between the subdomains. The simulation box is replicated in a direction perpendicular to the heat flux to enable periodic boundary conditions (PBCs) in three dimensions. Note that the variables $r_{i}$ and $v_{i}$ denote the position and velocity vectors, respectively, of i-th atom, whereas $v_{\Gamma_{r}}$ and $v_{\Omega}$ represent the center of mass velocities of the regions $\Gamma_{r}$ and the box, respectively.

The temperature gradient calculation is performed by dividing the simulation box into equal slabs in order to capture the spatial variation of the temperature. The instantaneous kinetic temperature $\mathrm{T}_{j}$ of slab $\mathrm{j}$ is given by

$$
T_{j}=\frac{2}{\left(N_{j} f-3\right) K_{b}} \sum_{i \in \text { binj }}^{N}\left[\frac{1}{2} m_{i}\left(v_{i}^{2}-v_{\Gamma_{i}}^{2}\right)\right]
$$




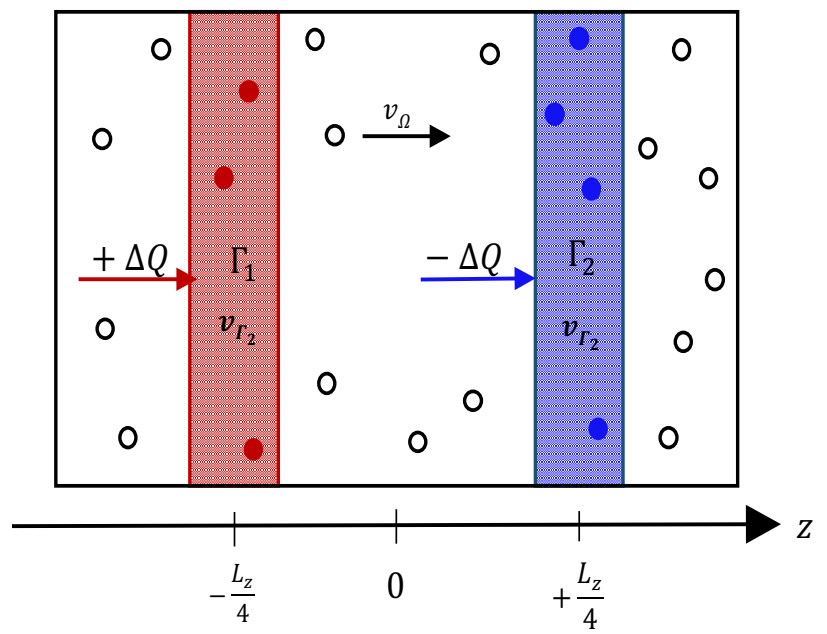

Figure 4.1: Illustration of the simulation box, $\Omega$, with a hot reservoir, $\Gamma_{1}$ (red), and a cold reservoir, $\Gamma_{2}$ (blue). The center of mass velocities of $\Omega, \Gamma_{1}$, and $\Gamma_{2}$ are $v_{\Omega}, v_{\Gamma 1}$, and $v_{\Gamma 2}$, respectively.

where $K_{b}$ represents Boltzmanns constant and $N_{j}$ represents the number of particles contained in slab $j$. The quantity $f$ represents the number of degrees of freedom per atom. Particles outside any thermostatted region are not affected by the velocity rescaling. For the individual region $\Gamma_{k}$ the velocity update for the particles located in $\Gamma_{r}$ can be formulated as

$$
v_{i} \longmapsto \bar{v}_{i}=\xi_{k} v_{i}+\left(1-\xi_{k}\right) v_{\Gamma_{k}}
$$

where the rescaling factor is defined as:

$$
\xi_{k}=\sqrt{\left(1+\frac{\Delta Q_{\Gamma_{k}}}{k_{\Gamma k}}\right)}
$$

with $k_{\Gamma k}$ representing the non-translational kinetic energy and $\Delta Q_{\Gamma k}$ representing the amount of heat added or subtracted in each subdomain. In their study, Wirnsberger et al. 58 considered a coordinate correction to overcome the energy loss as follows:

$$
\mathbf{x}(\Delta t)-\Delta t^{3} \epsilon \mathbf{x}_{e x}(0)=\mathbf{x}_{e x}(\Delta t)+O\left(\Delta t^{4}\right)
$$

They ignored the additional velocity Verlet truncation error and all other correction terms af- 
fecting velocities only. Basically, the accuracy of the numerical approximation was enhanced by applying the correction $\Delta t^{3} \epsilon \mathbf{x}_{e x}(0)$ to the original solution. Their analysis yielded to the eHEX algorithm, whose steps are outlined in the following updated sequence:

$$
\begin{aligned}
v_{i}^{n} & =\xi_{k r_{i}}^{n} v_{i}^{n}+\left(1-\xi_{k r_{i}}^{n}\right) v_{\Gamma_{k r_{i}}} \\
v_{i}^{n+1 / 2} & =\bar{v}_{i}^{n}+\frac{\Delta t}{2 m_{i}} f_{i}^{n} \\
r_{i}^{n+1} & =r_{i}^{n}+\Delta t \bar{v}_{i}^{n+1 / 2} \\
f_{i}^{n+1} & =-\left.\nabla_{r_{i}} U(r)\right|_{r=r^{n+1}} \\
v_{i}^{n+1} & =\bar{v}_{i}^{n+1 / 2}+\frac{\Delta t}{2 m_{i}} f_{i}^{n+1} \\
v_{i}^{n+1} & =\bar{\xi}_{k\left(\bar{r}_{i}\right)}^{n+1} \bar{v}_{i}^{n+1}+\left(1-\bar{\xi}_{k\left(\bar{r}_{i}\right)}^{n+1}\right) \bar{v}_{\Gamma_{k\left(r_{i}\right)}^{n+1}}
\end{aligned}
$$

where the correction term was given by

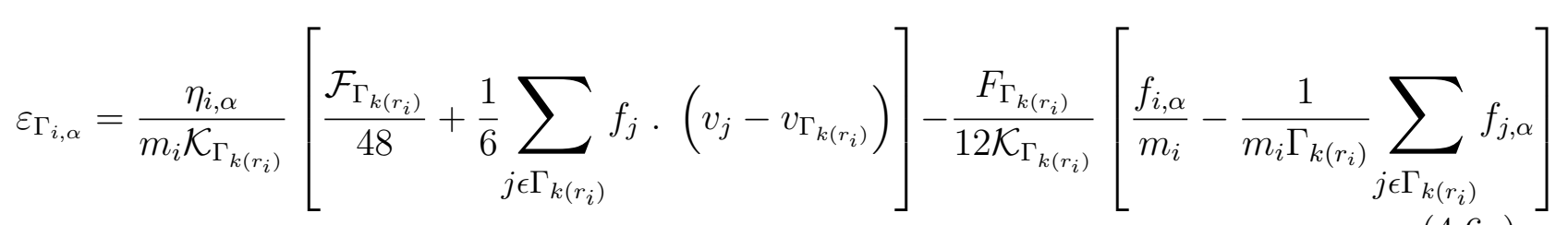

The symbols used in Eqs.(4.6) are described in the nomenclature. Further details of the eHEX scheme used in this work can be found in [58.

\subsubsection{Force Field Model}

The n-alkanes $\left(\mathrm{nC}_{5}-\mathrm{nC}_{10}\right)$ were represented using a modified version of Martin and Siepmann [60] TraPPE-UA force field model proposed by Makrodimitri et al. [67]. As mentioned earlier, TraPPE-UA has been validated to be very accurate for thermodynamic properties of n-alkanes in the pure state and in mixtures over a wide range of conditions [64, 67]. It was optimized to reproduce accurately the liquid-vapor coexistence curve and predict the critical properties for several linear and branched alkanes. In the original TraPPE-UA, all pseudo atoms along the alkane chain are connected with fixed bonds. However, Makrodimitri et al. 
[67] used instead a harmonic bond stretching potential in order to predict accurately system densities and diffusion coefficients in hydrocarbon mixtures.

The potential energy functions and parameters used in the augmented TraPPE-UA model are summarized in Table 4.1). The potential energy model accounts for bond stretching,

Table 4.1: Potential energy functions and parameters for the TraPPE UA model

\begin{tabular}{|c|c|c|}
\hline & Potential energy functions & Potential energy parameters \\
\hline $\begin{array}{l}\text { Non- } \\
\text { bonded LJ }\end{array}$ & $U_{\text {non-bonded }}=\sum_{\text {pairs }} 4 \varepsilon_{i j}\left[\left(\frac{\sigma_{i j}}{r_{i j}}\right)^{12}-\left(\frac{\sigma_{i j}}{r_{i j}}\right)^{6}\right]$ & 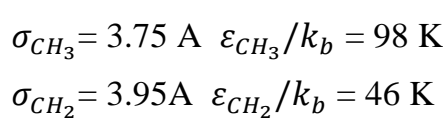 \\
\hline $\begin{array}{l}\text { Bond } \\
\text { stretching }\end{array}$ & $U_{\text {stretch }}=\sum_{\text {bonds }} \frac{k_{r}\left(r-r_{e q}\right)^{2}}{2}$ & $\begin{array}{l}k_{r} / k_{b}=96500 \mathrm{~K} / \mathrm{A}^{2} \\
r_{e q}=1.54 \mathrm{~A}\end{array}$ \\
\hline $\begin{array}{l}\text { Bond-angle } \\
\text { bending }\end{array}$ & $U_{\text {bend }}=\sum_{\text {angles }} \frac{k_{\theta}\left(\theta-\theta_{e q}\right)^{2}}{2}$ & $\begin{array}{l}k_{\theta} / k_{b}=62500 \mathrm{~K} / \mathrm{rad}^{2} \\
\theta_{e q}=114^{\circ}\end{array}$ \\
\hline Torsion & $U_{\text {tors }}=\sum_{\text {dihedrals }}\left[\begin{array}{c}c_{0}+c_{1}(1+\cos \emptyset) \\
+c_{2}(1-\cos 2 \emptyset) \\
+c_{3}(1+\cos 3 \emptyset)\end{array}\right]$ & $\begin{array}{l}c_{0} / k_{b}=0 \mathrm{~K} \\
c_{1} / k_{b}=355.03 \mathrm{~K} \\
c_{2} / k_{b}=-68.19 \mathrm{~K} \\
c_{3} / k_{b}=791.32 \mathrm{~K}\end{array}$ \\
\hline
\end{tabular}

bond bending, torsional rotation, and van der Waals non-bonded interactions. The nonbonded intermolecular interactions are modeled with a pairwise Lennard-Jones (LJ) potential with a cutoff length of $14 \mathrm{~A}$. Intramolecular non-bonded interactions for sites separated by more than three bonds are also described by a LJ potential. The standard LorentzBerthelot (LB) combining rules have been used extensively in previous simulation work to describe nonbonded LJ interactions between sites of different types $\mathrm{i}$ and $\mathrm{j}$ according to the expressions $\varepsilon_{i j}=\sqrt{\left(\varepsilon_{i i} \varepsilon_{j j}\right)}$ and $\sigma_{i j}=\left(\sigma_{i i}+\sigma_{j j}\right) / 2$ where $\varepsilon_{i i}$ and $\sigma_{i i}$ are the parameters for interactions between identical sites [67, 68]. Although phase equilibrium and thermodiffusion magnitude can be very sensitive to the combining rules, LB proved to be accurate when modeling nalkanes-n-alkanes mixtures with UA [56, 68]. Therefore, the LB method was entailed in this work to obtain unlike-pair parameters. The bond stretching and angle bending potentials 
were described with harmonic functions. The torsional dihedral angle potential function was defined by using the Optimized Intermolecular Potential Functions (OPLS). Details on the force field functional form and parameters for the various terms of n-alkanes can be found in 67 .

\subsection{Methodology and Numerical Computations}

Atomistic molecular dynamics simulations require a starting model to generate the system initial configuration. Packmol was used in order to pack the molecules in a cubic simulation box and generate atoms initial coordinates [41. The interaction force field model was created using a cross-platform molecular builder Moltemplate [69]. Subsequently, EMD and NEMD simulations were carried out within the MD simulator Large-scale Atomic/Molecular Massively Parallel Simulator (LAMMPS) [30]. The calculated values of transport properties resulting from MD simulations highly depend on the system size, the intensity of perturbations, and the simulation time length [70, 71]. In order to secure reliable results, these numerical parameters were monitored until a consistency was observed.

\subsubsection{EMD Simulations}

To test the validity of the force field model, EMD simulations were conducted to obtain thermodynamics equilibrium properties (density and self-diffusion coefficient). Simulations were carried out at 300.15 K and 0.1 MPa, and Periodic Boundary Conditions (PBCs) were applied for all systems. To simulate the systems with just one component, 1000 molecules were used. On the other hand, for the binary mixtures, a total of 2000 molecules were included in the systems. In order to avoid highly overlapped atoms, the system configuration was initially brought to a local potential energy minimum by performing an energy minimization. Next, an annealing procedure was used in order to accelerate the equilibration and avoid trapping in a local minimum of energy: the system was initially heated up to $700 \mathrm{~K}$ and then cooled down back to $300.15 \mathrm{~K}$. Annealing process is important before performing equilibration runs since it allows the system to avoid high energy barriers that force energy minimization into local minima. Also, it is necessary to apply the appropriate rate of heating and cooling during the annealing process that will bring the system to a global minimum. Next, the NPT ensemble with a Nose Hoover thermostat and barostat using the time con- 
stants of 0.1 and $1 \mathrm{ps}$, respectively, was employed in order to determine the equilibrium system density at a target pressure of $0.1 \mathrm{MPa}$. Long-range tail corrections to the energy and pressure were included [29]. The equations of motion were integrated using the velocity Verlet algorithm, and a time step of 1 fs was used in all simulation runs.

The diffusion coefficients of pure components were obtained from EMD simulations in the NVT ensemble using the Nose Hoover thermostat at the previously determined equilibrium density corresponding to a pressure of $0.1 \mathrm{MPa}$. After running NVT for $2 \mathrm{~ns}$, the average system energy was calculated using the NVE ensemble. The energy of the last configuration of the NVT simulation was adjusted by carrying out an additional NVE equilibration run for 5 ns. This procedure allowed us to achieve an average equilibrium temperature of $300.15 \pm$ $2 \mathrm{~K}$. The final configuration obtained was used as the initial configuration in the simulations for determining the diffusion coefficient, which is also performed again in the NVE ensemble with an additional run time of 5 ns. The atoms configurations were written out every 200 time steps. The self-diffusion coefficients $D_{\text {self }}$ of pure components were calculated via the Einstein relation [67, 71] as

$$
D_{i, s e l f}=\frac{1}{2 d N} \lim _{k \rightarrow \infty} \frac{1}{k \Delta t}\left\langle\sum_{i=1}^{N}\left(\mathbf{r}_{i}(t+k \Delta t)-\mathbf{r}_{i}(t)\right)^{2}\right\rangle
$$

where $\mathbf{r}_{i}(t)$ is the particle position vector of type $\mathrm{i}$ at time $\mathrm{t}$ and $\left(\mathbf{r}_{i}(t+k \Delta t)-\mathbf{r}_{i}(t)\right)^{2}$ represents the mean square displacement of the center of mass of molecules. $N$ denotes the total number of molecules and $\Delta t$ denotes the time step. The quantity $d(=3)$ is a numerical constant which depends on system dimensions. The simulation time $(t)$ for all diffusion coefficient determinations was large enough to achieve a constant slope behavior of the mean square displacement. EMD results from density and the diffusion coefficient were used to test the accuracy of the force field in the determination of this transport property by comparison with experimental data and other simulation results available with other force fields. 


\subsubsection{BD-NEMD Simulations}

The system size and strength of the heat flux were considered as two important parameters to achieve good computational results and local equilibrium, as defined by the validity of thermodynamic equations [42, 70]. According to Zhang and Muller-Plathe [43], a stronger perturbation produces better results due to a better signal to noise ratio, provided the linear response holds. Whereas, a weaker temperature gradient causes the average composition in each slab to be of the same order of magnitude as the fluctuations, thus resulting in an inaccurate estimation of Soret coefficients. Therefore, the magnitude of the heat flux was regulated such that the imposed temperature gradient is high enough to ensure a linear response with a quantifiable signal to noise ratio and low enough to avoid phase transition and deviation from equilibrium caused by high perturbations of particles motion inside the thermostatted regions (by analyzing the density gradient) [42, 43]. The criteria of validity of local equilibrium from NEMD, clearly formulated in the work of Kjelstrup et al. [42], were checked in order to support the consistency of our computational results under extreme temperature gradients.

In order to ensure a good statistical precision, all NEMD binary systems contained 2000 molecules (given composition). With the previously obtained densities, binary mixtures configurations were equilibrated again using NVT and NVE and a time step of 1 fs. The system region was modified for BD-NEMD simulations. The box dimensions were rescaled such that $L_{z} / 2=L_{x}=L_{y}$, while keeping the equilibrated system density constant. In order to capture the spatial variation of the temperature and composition, the simulation box was divided in the $\mathrm{z}$ direction into 32 bins of equal size. The number of molecules inside each slab (around 62 molecules) was considered large enough for it to behave as a thermodynamic system [42, 70]. A unidirectional heat flux was imposed on the system along the z-axis. The two thermostat regions (consisted each of 4 slabs) were centered at the points $z= \pm L_{z} / 4$ (see Figure 4.1). During each time step, the heat $\Delta Q$ is taken from region 2 and added to region $1\left(\Delta Q_{\Gamma_{1}}=\Delta Q_{\Gamma_{1}}=\Delta Q>0\right)$ maintaining a fixed energy flux into the reservoir $\mathcal{F}=\Delta Q_{\Gamma} / \Delta t$. Each BD-NEMD simulation (given composition) was at least $45 \times 10^{6}$ time steps long (after an equilibrium simulation of $5 \times 10^{6}$ time steps) with a larger time step of 2 fs for which the total system energy exhibited a minor drift (less than $0.3 \%$ ). Since the problem can be described as a 1D diffusion equation, the minimum 
expected time for concentration and temperature gradients to attain stationary state can be estimated as the same order as $L_{z}^{2} /(4 D) \sim 5.3 \mathrm{~ns}$ and $L_{z}^{2} /\left(4 T D_{T}\right) \sim 5 \mathrm{~ns}$, respectively. The latter asserts that the total run time of our simulations is considered large enough to reach a steady state and obtain linearity of both composition and temperature gradients. The first $2.5 \times 10^{6}$ time steps of the simulation were discarded from the average calculations to avoid transient effects. Local temperature and density fraction were computed at each of the 500 time steps, and averages of 20 samples were dumped every 10000 time steps to a file for later analysis. In addition, accumulated averages of the system overall energy and temperature and the local mole fraction and temperature for each layer were computed [72]. The corresponding gradients were also calculated from a linear fit of the local quantities. The hot and cold regions (4 slabs each) as well as two neighbor slabs have been discarded from the temperature gradient measurement because of non-linear behavior in these layers (see Figure 4.4 [43]. The statistical errors were estimated from the standard deviation from the mean.

\subsection{Results and Discussions}

\subsubsection{Validation of the Force Field Model}

As summarized in Table 4.2, the computational results of density at $300.15 \mathrm{~K}$ and $0.1 \mathrm{MPa}$ of the single component fluids as well as the three binary mixtures of $\mathrm{nC}_{5}-\mathrm{nC}_{10}$ showed good agreement with the experimental data. Typically, the agreement is better than $1 \%$ for all mixture compositions. The diffusion coefficients of pure components were also reported and compared with experimental and some simulation data of other force fields available in [73]. As seen from Table 4.3 , the self diffusion coefficients of $\mathrm{nC}_{5}$ and $\mathrm{nC}_{10}$ obtained from this work were in very good agreement with the experimental data with an error of $8.1 \%$ and $3.42 \%$, respectively. They also revealed a better performance with respect to the AUA model. These results support the employment of the augmented TraPPE-UA force field and confirm its precision in predicting thermodynamics equilibrium properties for n-alkanes liquid pure and binary mixtures. Note that the data extracted from Ref.[73] were interpolated to get values at the same temperature of $300.15 \mathrm{~K}$ and the statistical errors for experimental densities and self diffusion coefficients were estimated from the standard deviation from the mean using uncorrelated data [74]. 
Table 4.2: Densities of $\mathrm{nC}_{5}-\mathrm{nC}_{10}$ mixture for different molar composition at $300.15 \mathrm{~K}$ and $0.1 \mathrm{MPa}$. The $\%$ error $\Delta$ of the numerical density of this work was computed with respect to NIST data

\begin{tabular}{cccc}
\hline \hline \multirow{2}{*}{$\mathrm{x}_{\mathrm{C} 10}$} & \multicolumn{3}{c}{ Mixture Density $\left(\mathbf{K g} / \mathbf{m}^{\mathbf{3}}\right)$} \\
& NIST & This work & $\Delta(\%)$ \\
\hline 0 & 618.84 & 619.25 & 0.07 \\
0.2 & 652.40 & 653.71 & 0.20 \\
0.5 & 688.32 & 690.21 & 0.27 \\
0.8 & 712.96 & 715.13 & 0.30 \\
1 & 724.97 & 727.54 & 0.35 \\
\hline \hline
\end{tabular}

Table 4.3: Potential energy functions and parameters for the TraPPE UA model

\begin{tabular}{ccccc}
\hline \hline & & \multicolumn{2}{c}{$\boldsymbol{D}_{\text {self }}\left(\mathbf{1 0}^{-9} \mathbf{~ m}^{\mathbf{2}} / \mathbf{s}\right)$} \\
& Expt. & TraPPE-UA* & AUA1 & AUA2 \\
\hline $\mathrm{nC}_{5}$ & 5.68 & $6.14(8.1)$ & $6.21(9.33)$ & $6.27(10.39)$ \\
$\mathrm{nC}_{10}$ & 1.46 & $1.51(3.42)$ & $1.52(4.11)$ & $1.7(16.44)$ \\
\hline \hline
\end{tabular}

\subsubsection{Linearity and Convergence of Composition and temperature profiles}

Density, temperature, and concentration profiles for all $\mathrm{nC}_{5}-\mathrm{nC}_{10}$ mixtures clearly show a linear trend (see Figure 4.2). 

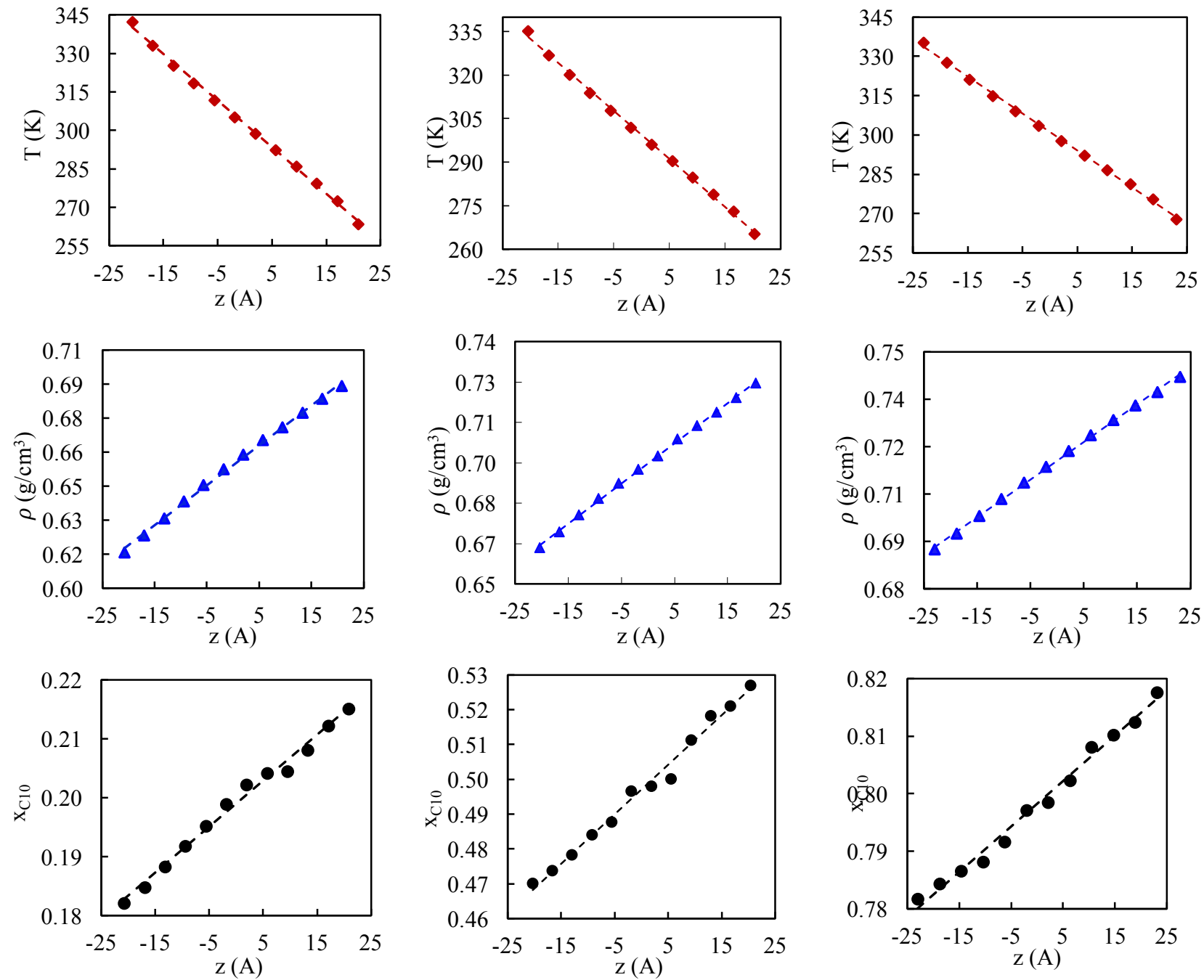

(a)

(b)

(c)

Figure 4.2: Temperature (top), density (middle), and $\mathrm{nC}_{10}$ mole fraction (bottom) profiles for the three different molar compositions of $\mathrm{nC}_{5}-\mathrm{nC}_{10}$ : (a) $x_{C 10}=0.2$, (b) $x_{C 10}=0.5$, and (c) $x_{C 10}=0.8$ 
The continuity of the three gradients indicates that the system behavior, despite strong thermal gradients (highest value of $1.79 \times 10^{1} 0 \mathrm{~K} / \mathrm{m}$ attained for $x_{C 10}=0.2$ ), is still in the domain of linear response. To support this assumption, the criteria of local equilibrium from NEMD were checked.39 Each layer contained around 62-63 molecules. The temperature and mole fraction relative fluctuations were minimal averaging $0.35 \%$ and $4 \%$ in each slab, respectively. The relative change of temperature and density across the slab in the direction of diffusion was smaller than one. The linearity condition of both gradients was also evaluated through the R2, which achieved a value higher than 0.98 and 0.99 for composition and temperature, respectively (Figure 4.3). 

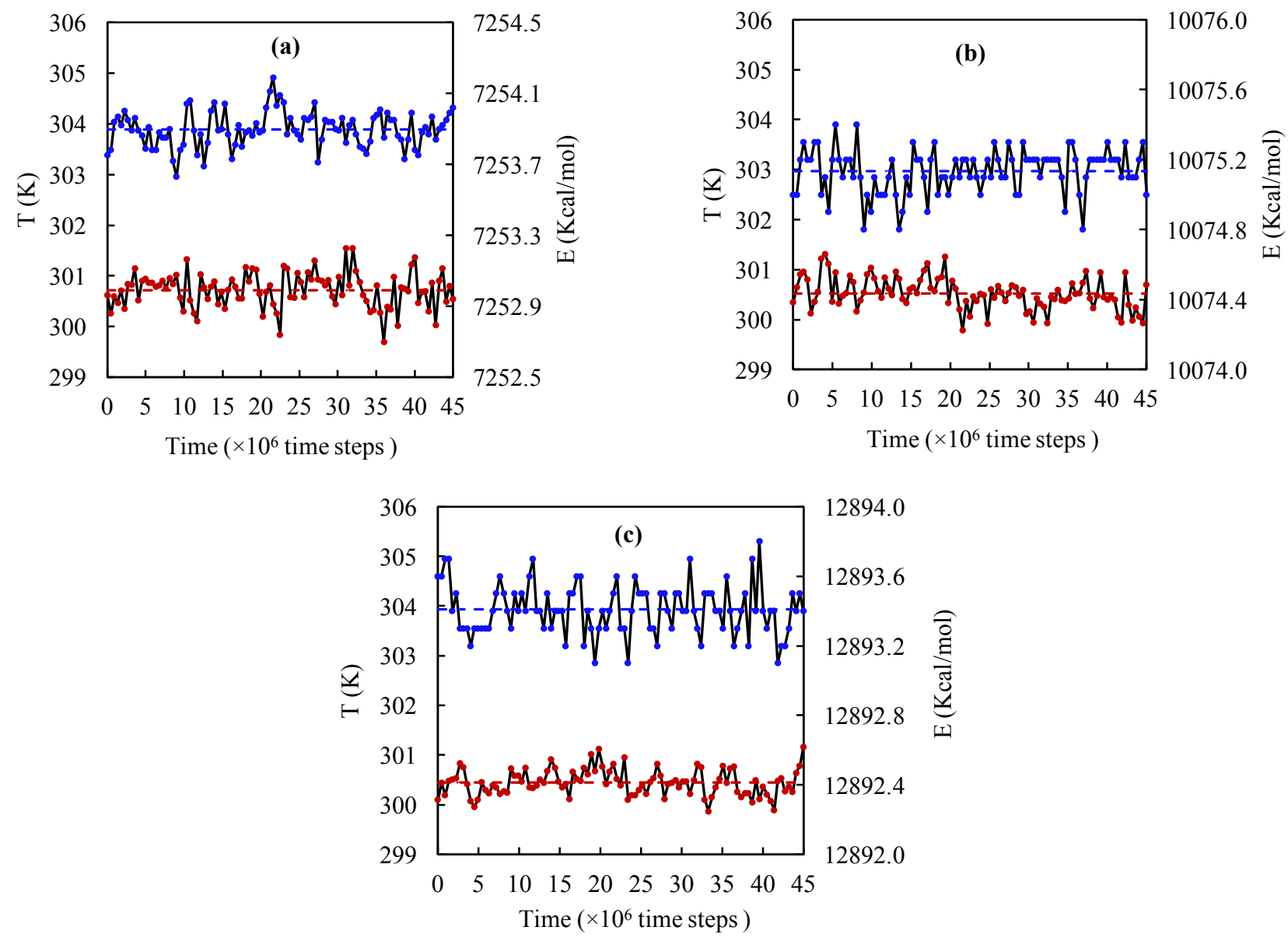

Figure 4.3: System overall temperature (red) and energy (blue) variation as a function of simulation time for the three different molar compositions of $\mathrm{nC}_{5}-\mathrm{nC}_{10}$ : (a) $x_{C 10}=0.2$, (b) $x_{C 10}=0.5$, and (c) $x_{C 10}=0.8$. 
Low and high temperatures were detected in the coldest and hottest slabs, respectively. The lowest temperature attained in the cold region was $250 \mathrm{~K}$ (see Figure 4.4), which is above the experimental melting points of $\mathrm{nC}_{5}$ and $\mathrm{nC}_{10}$ (143.4 and $243.3 \mathrm{~K}$, respectively). The latter indicates that the mixture did not cross the barrier to act as a supercooled liquid or to affect the dynamics of the system by slowing down the particles movement. On the other hand, the hot region reached higher temperatures than the vapor-liquid equilibrium region for the $\mathrm{nC}_{5}-\mathrm{nC}_{10}$ mixture. According to experimental data, the boiling points of $\mathrm{nC}_{5}$ and $\mathrm{nC}_{10}$ are 309.2 and $447.2 \mathrm{~K}$, respectively.
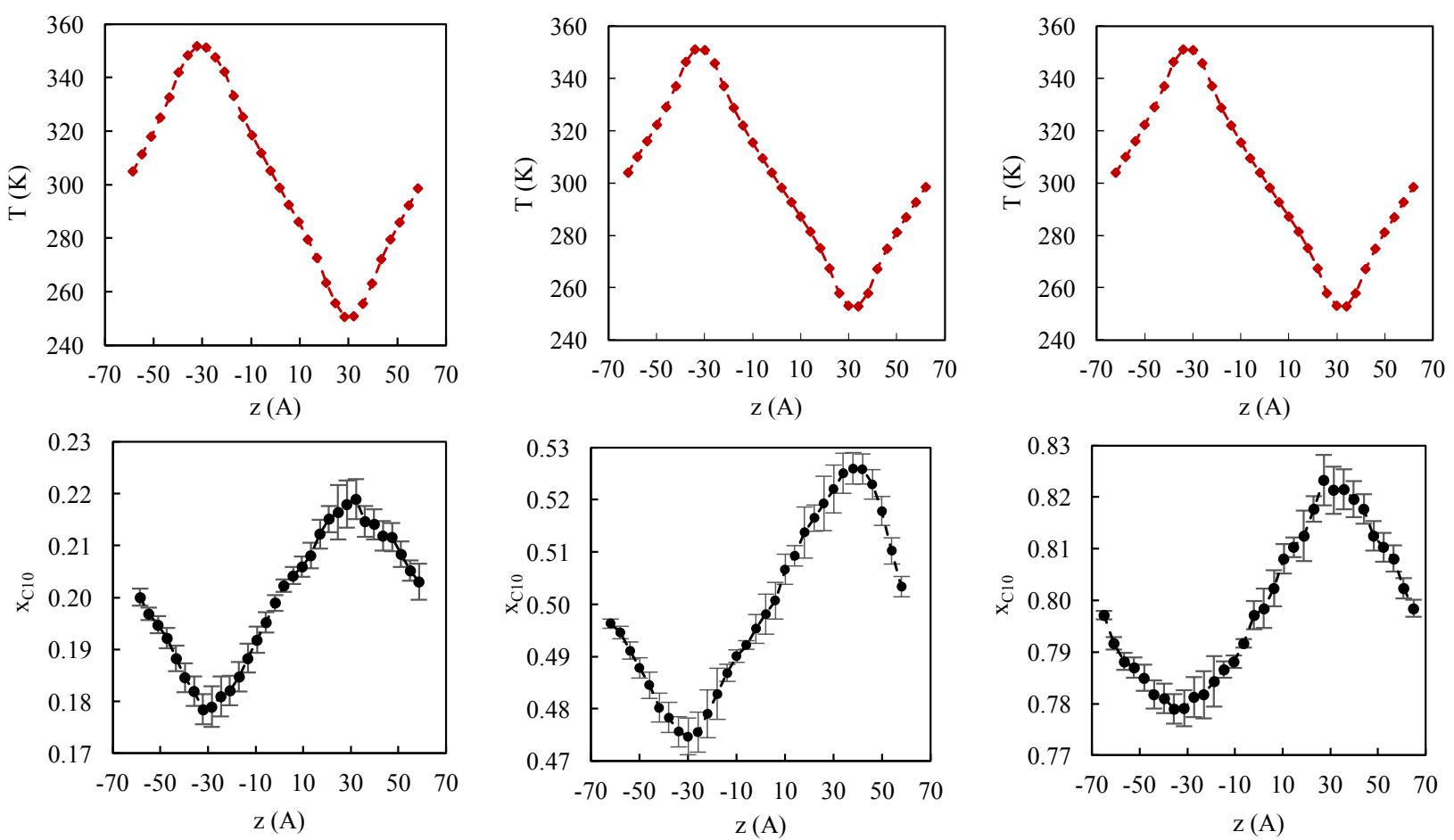

(a)

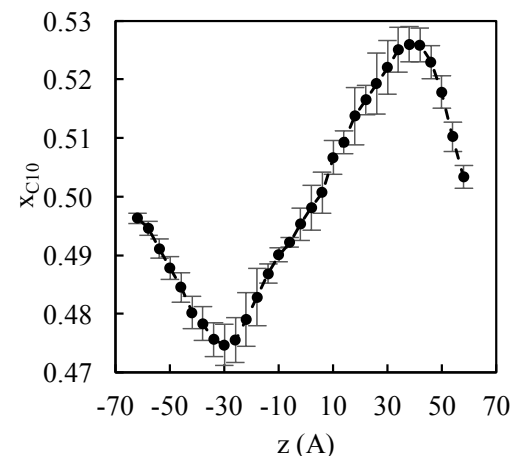

(b)

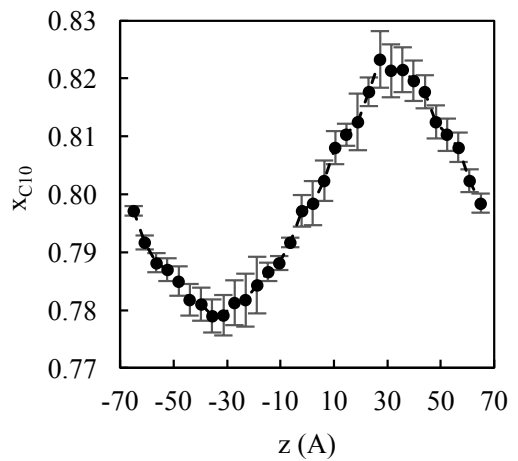

(c)

Figure 4.4: Temperature (top) and $\mathrm{nC}_{10}$ mole fraction (bottom) profiles for the three different molar compositions of $\mathrm{nC}_{5}-\mathrm{nC}_{10}$ : (a) $x_{C 10}=0.2$, (b) $x_{C 10}=0.5$, and (c) $x_{C 10}=0.8$. 
Figure 4.4 indicates that the temperature in the hottest slab reached a value around $351 \mathrm{~K}$ (for $x_{C 10}=0.2$ ), which is higher than the boiling point of $\mathrm{nC}_{5}$. Zhang and MullerPlathe [43] observed a similar behavior for a benzene/cyclohexane mixture at $324 \mathrm{~K}$ and mole fraction of 0.25 (with a temperature gradient of the order of $10^{10} \mathrm{~K} / \mathrm{m}$ ). They observed a temperature around $360 \mathrm{~K}$ in the hot slab, which is higher than the boiling point of both substances (353.1 $\mathrm{K}$ for benzene and $353.7 \mathrm{~K}$ for cyclohexane), indicating a possible vapor phase transition. To rule out the formation of a vapor phase, Zhang and Muller-Plathe [43] tested the linearity of the density profile across the simulation box to make sure that the mixture behaves as a liquid in each slab.

In order to confirm the liquid-like behavior of this work, the $\mathrm{nC}_{5}-\mathrm{nC}_{10}$ mixture, the steady state density profile was plotted for all mixtures. Figure 4.5 shows that even with a large imposed heat flux, the average density varies linearly through the system and the simulated mixture behaved as a liquid in every slab.
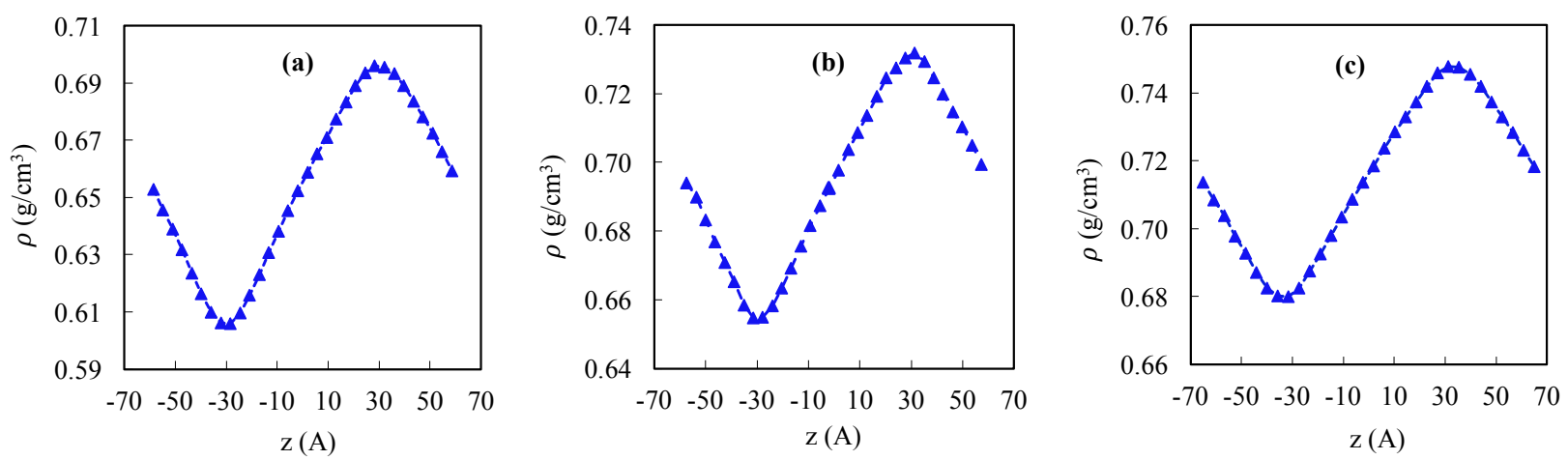

Figure 4.5: System density profile for the three different molar compositions of $\mathrm{nC}_{5}-\mathrm{nC}_{10}$ : (a) $x_{C 10}=0.2$, (b) $x_{C 10}=0.5$, and (c) $x_{C 10}=0.8$. 
The time evolution of thermal and composition gradients across the simulation box were plotted in order to check for convergence. Figure 4.6 shows that the time needed for the temperature gradient to reach a plateau (which was around $2 \times 10^{6}$ time steps)was significantly shorter than that of the concentration gradient or number density (a minimum of $20 \times 10^{6}$ time steps). The lines are running averages since the start of the production run. Additionally, Figure 4.6 illustrates a relatively higher fluctuation in the number density gradient. These fluctuations can be due to the size of the molecules and their movement: counting the number of center of masses in each slab can be very different for each time step.

It was noticed that the $\mathrm{R} 2$ value of concentration profiles reached an acceptable value (higher than 0.98) before attaining the steady state. In order to avoid the errors due to composition fluctuations and check for exact convergence of the composition gradient, the simulation time for all the cases was extended to at least $55 \times 10^{6}$ time steps. According to 4.6, the number density gradient time evolution established nearly a plateau for the larger simulation, which indeed reduced the uncertainties of Soret coefficient calculations. The errors in composition and temperature in each slab were taken into account to compute profiles and their uncertainties. The standard error of the Soret coefficient was computed with the composition and temperature profile uncertainties using standard error propagation methods [74].
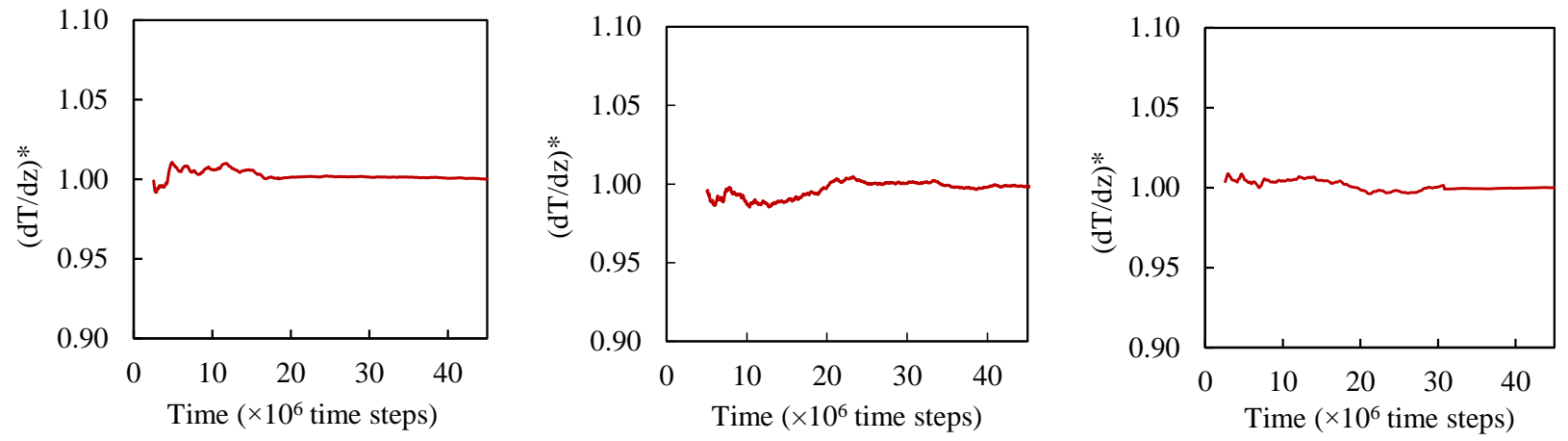

Figure 4.6: Temperature (top) and number density of $\mathrm{nC}_{10}$ (bottom) gradients variation over simulation time for the three different molar compositions of $\mathrm{nC}_{5}-\mathrm{nC}_{10}$ : (a) $x_{C 10}=0.2$, (b) $x_{C 10}=0.5$, and (c) $x_{C 10}=0.8$. 


\subsubsection{Comparison of $S_{T}$ with experimental and other numerical work}

The experimental Soret coefficient, $S_{T}$, has been determined as a combination of the experimental data of thermal diffusion $\left(D_{T}\right)$ and molecular diffusion $(D)$ coefficients obtained from the literature [44, 75, 76]. The average value of the selected data measured using different techniques was considered as a benchmark reference for numerical comparison and validation (see Table 4.4). All the coefficients of the $\mathrm{nC}_{5}-\mathrm{nC}_{10}$ mixture were all measured under the same conditions used in our numerical simulations for the three nC10 molar fractions: (a) $x_{C 10}=0.2$, (b) $x_{C 10}=0.5$, and (c) $x_{C 10}=0.8$.

Table 4.4: Experimental data of diffusion $(D)$ and thermal diffusion coefficients $\left(D_{T}\right)$ measured using different techniques at $300.15 \mathrm{~K}$ for the $\mathrm{nC}_{5}-\mathrm{nC}_{10}$ mixture for three different molar compositions.

\begin{tabular}{lccc}
\hline \hline $\mathrm{x}_{\mathrm{C} 10}$ & 0.2 & 0.5 & 0.8 \\
\hline & \multicolumn{3}{c}{$D / 10^{-5}\left(\mathrm{~cm}^{2} / \mathrm{s}\right)$} \\
TDFRS & 2.63 & 2.30 & 1.90 \\
SST & 3.36 & 2.96 & 2.20 \\
Average & 3.00 & 2.63 & 2.05 \\
& \multicolumn{3}{c}{$D_{T} / 10^{-8}\left(\mathrm{~cm}^{2} / \mathrm{s} . \mathrm{K}\right)$} \\
TDFRS & $9.28 \pm 0.75$ & $7.54 \pm 0.61$ & $7.18 \pm 0.59$ \\
TC para. & $10.49 \pm 0.30$ & $8.76 \pm 0.30$ & $6.76 \pm 0.20$ \\
TC cyl. & $10.36 \pm 0.50$ & $8.67 \pm 0.40$ & $6.92 \pm 0.30$ \\
TDFRS & $10.81 \pm 0.70$ & $9.11 \pm 0.60$ & $7.75 \pm 0.60$ \\
Average & $10.24 \pm 0.70$ & $8.52 \pm 0.60$ & $7.15 \pm 0.60$ \\
& \multicolumn{3}{c}{$S_{T}=D_{T} / D / 10^{-3}\left(\mathrm{~K}^{-1}\right)$} \\
Average & $3.42 \pm 0.56$ & $3.24 \pm 0.48$ & $3.49 \pm 0.42$ \\
\hline \hline
\end{tabular}

MD simulations were first conducted using the original BD-NEMD (HEX) method developed by Hafskjold et al. [38] in order to verify the choice of the augmented TraPPE-UA force field over other potential models that have also been combined with BD-NEMD algorithm to estimate thermodiffusion of the $\mathrm{nC}_{5}-\mathrm{nC}_{10}$ binary mixture. The results of $S_{T}$ of the original 
HEX along with the augmented TraPPE-UA and the numerical data of other force field models in the literature were first compared to the benchmark experimental values. Table 4.5 summarizes the values and the percentage deviation of the different force field models with respect to the experimental results evaluated for the three molar compositions of the of $\mathrm{nC}_{5}-\mathrm{nC}_{10}$ mixture [(a) $x_{C 10}=0.2$, (b) $x_{C 10}=0.5$, and (c) $x_{C 10}=0.8$. When compared to the other models, the augmented TraPPE-UA presented the least deviation (see Table 4.5). The Smit-Karaborni-Siepmann (SKS) showed a discrepancy up to 30\%. The LJ sphere model implemented by Galliero et al. [59] revealed a deviation up to $20 \%$ compared to the benchmark values. Whereas, the values obtained by Mie Chain Coarse- Grained (MCCG) [39] were off by nearly 11\%-25\% depending on the mixture molar composition. In other words, the comparison emphasizes that the augmented TraPPE-UA potential force field over other potential models that have also been combined with BD-NEMD algorithm to estimate thermodiffusion of the $\mathrm{nC}_{5}-\mathrm{nC}_{10}$ binary mixture. The results of $\mathrm{ST}$ of the original HEX along with the augmented TraPPE-UA and the numerical data of other force field models in the literature were first compared to the benchmark experimental values. Table 4.5 summarizes the values and the percentage deviation of the different force field models with respect to the experimental results evaluated for the three molar compositions of the $\mathrm{nC}_{5}-\mathrm{nC}_{10}$ mixture [(a) $x_{C 10}=0.2$, (b) $x_{C 10}=0.5$, and (c) $x_{C 10}=0.8$. When compared to the other models, the augmented TraPPE-UA presented the least deviation (see Table 4.5). The Smit-Karaborni-Siepmann (SKS) showed a discrepancy up to 30\%. The LJ sphere model implemented by Galliero et al. [6] revealed a deviation up to $20 \%$ compared to the benchmark values. Whereas, the values obtained by Mie Chain Coarse- Grained (MCCG) [39] were off by nearly $11 \% 25 \%$ depending on the mixture molar composition. In other words, the comparison emphasizes that the augmented TraPPE-UA potential field is a very good choice to reproduce the real molecular interactions in the system and predict thermodiffusion transport properties. In order to assess the performance of the eHEX method in predicting the thermodiffusion factor, results were compared to the original HEX. Both approaches were applied along with the augmented TraPPE-UA. As seen in Table 4.6, the data from this study eHEX show a very good agreement with the experimental data. More precisely, the accuracy in predicting the Soret coefficient was improved compared to the original HEX method with corresponding deviations from experimental between approximately $0.55 \%$ and $6.5 \%$, depending upon the composition of the mixture. From the results of all the mixture compositions analyzed so far, it can be concluded that the eHEX algorithm combined with 
Table 4.5: Numerical values of ST computed using BD-NEMD with different force field (FF) models for the the $\mathrm{nC}_{5}-\mathrm{nC}_{10}$ mixture at three different molar compositions. The values in the brackets represent the percentage error with respect to the benchmark values. HEX along with augmented TraPPE-UA* correspond to this work results.

\begin{tabular}{|c|c|c|c|c|}
\hline \multicolumn{2}{|c|}{$\mathrm{x}_{\mathrm{C} 10}$} & 0.2 & 0.5 & 0.8 \\
\hline & & \multicolumn{3}{|c|}{$\mathrm{S}_{\mathrm{T}} / 10^{-3}\left(\mathrm{~K}^{-1}\right)$} \\
\hline \multicolumn{2}{|c|}{ Expt. Avg. } & $3.42 \pm 0.56$ & $3.24 \pm 0.48$ & $3.49 \pm 0.42$ \\
\hline NEMD & FF-Model & & & \\
\hline \multirow{4}{*}{$\begin{array}{l}\text { BD-NEMD } \\
\text { (HEX/PEX) }\end{array}$} & SKS & $\begin{array}{c}2.81 \pm 0.42 \\
-17.77\end{array}$ & $\begin{array}{c}2.91 \pm 0.45 \\
-10.17\end{array}$ & $\begin{array}{c}2.49 \pm 0.58 \\
-28.63\end{array}$ \\
\hline & LJ sphere & $\begin{array}{c}4.10 \pm 0.49 \\
-19.98\end{array}$ & $\begin{array}{c}3.13 \pm 0.37 \\
-3.38\end{array}$ & $\begin{array}{c}3.43 \pm 0.47 \\
-1.69\end{array}$ \\
\hline & MCCG & $\begin{array}{c}2.82 \pm 0.60 \\
-17.48\end{array}$ & $\begin{array}{c}2.88 \pm 0.40 \\
-11.1\end{array}$ & $\begin{array}{c}2.62 \pm 0.60 \\
\quad-24.91\end{array}$ \\
\hline & TraPPE-UA* & $\begin{array}{c}3.13 \pm 0.20 \\
-8.48\end{array}$ & $\begin{array}{c}3.01 \pm 0.20 \\
-7.1\end{array}$ & $\begin{array}{c}3.43 \pm 0.25 \\
-1.68\end{array}$ \\
\hline
\end{tabular}

the augmented TraPPE-UA force field model, proposed in this study, is a very good choice for studying thermodiffusion in n-alkanes liquid mixtures.

\subsection{Conclusion}

The performance of Boundary Driven Non-Equilibrium Molecular Dynamics (BD-NEMD) using the enhanced heat exchange (eHEX) algorithm was studied in order to test its ability to accurately predict thermodiffusion in $\mathrm{C}_{5}-\mathrm{nC}_{10}$ binary mixtures for three different molar compositions. The eHEX algorithm is an extended version of the HEX algorithm, developed by Hafskjold and co-workers with an improved energy conservation. It was applied along with the augmented TraPPE-UA force field. The Soret coefficients of the $\mathrm{C}_{5}-\mathrm{nC}_{10}$ mixture for the three mole fractions [(a) $x_{C 10}=0.2$, (b) $x_{C 10}=0.5$, and (c) $\left.x_{C 10}=0.8\right]$ were assessed at $300.15 \mathrm{~K}$ and $0.1 \mathrm{MPa}$ and compared to the experimental data. In order to assert the advantage of the augmented TraPPE-UA force field over other potential models, which have also been combined with the BD-NEMD algorithm, the original HEX combined with the 
Table 4.6: Numerical values of $S_{T}$ computed using HEX and eHEX along with augmented TraPPE-UA * for the $\mathrm{nC}_{5}-\mathrm{nC}_{10}$ mixture at three different molar compositions. The values in the brackets represent the percentage error with respect to the benchmark values.

\begin{tabular}{|c|c|c|c|c|}
\hline \multicolumn{2}{|c|}{$\mathrm{x}_{\mathrm{C} 10}$} & 0.2 & 0.5 & 0.8 \\
\hline & & \multicolumn{3}{|c|}{$\mathrm{S}_{\mathrm{T}} / 10^{-3}\left(\mathrm{~K}^{-1}\right)$} \\
\hline \multicolumn{2}{|c|}{ Expt. Avg. } & $3.42 \pm 0.56$ & $3.24 \pm 0.48$ & $3.49 \pm 0.42$ \\
\hline \multirow[t]{2}{*}{ NEMD } & FF-Model & & & \\
\hline & & $3.13 \pm 0.20$ & $3.01 \pm 0.20$ & $3.43 \pm 0.25$ \\
\hline HEX & TroPDE I $\triangle * *$ & -8.48 & -7.1 & -1.68 \\
\hline \multirow{2}{*}{ eHEX } & 11аГTL-UA & $3.25 \pm 0.20$ & $3.14 \pm 0.15$ & $3.47 \pm 0.23$ \\
\hline & & -4.91 & -3.1 & -0.55 \\
\hline
\end{tabular}

augmented TraPPE-UA simulations was first conducted and compared to the numerical data of other potential field models available in the literature. Subsequently, the performance of this study eHEX algorithm was compared to the original HEX model in predicting the Soret coefficient, while applying the same force field model. The following conclusions were drawn from the findings:

1. The round-off and truncation errors related with the modified integration scheme and computational precision of eHEX manage to significantly conserve the system energy and temperature with minor instabilities around the mean.

2. The continuity of the three gradients (temperature, composition, and mixture density) emphasizes that the system behavior, despite the extreme gradients, is still in the domain of linear response and converges with minimal computational errors.

3. Soret coefficients estimates from our model show a very good agreement with the benchmark experimental data. More precisely, the accuracy in predicting the ST was approximately between $0.55 \%$ and $6 \%$, depending upon the composition of the mixture.

4. The assessment of $S_{T}$ numerical values of this work with other MD approaches clearly underlines that transport properties highly depend on the accuracy of NEMD and force field algorithms. In other words, the potential field parameters should be accurate enough to reflect the real molecular interactions in the system and reproduce precise liquid properties. Additionally, the NEMD heat exchange algorithm should mimic the real experimental process and conditions with a great computational and integration 
scheme precision.

Given that our proposed model performed well for calculating the thermodiffusion factor of binary n-alkane mixtures, the eHEX algorithm along with the augmented TraPPEUA force field model serves as a highly promising method and a low-cost alternative for experiments to study thermodiffusion. 


\section{Chapter 5}

\section{Enhanced Molecular Dynamics Evaluation of Thermophobicity in Ternary Mixtures}

The formatting of this chapter is presented in the same style as of our recently published paper. The original publication appears as [16]: S. Antoun, M. Z. Saghir, and S. Srinivasan, "Composition effect on thermophobicity of ternary mixtures: An enhanced molecular dynamics method." As we demonstrated in Chapter 4, the eHEX method along with TraPPE-UA is a potentially powerful method to predict thermodiffusion properties of asymmetric alkanes mixtures. While the results showed a very good agreement with benchmarked experimental data, the simulated systems only consisted of binary hydrocarbons. In this chapter, we build off these results to study a more complex system comprising of ternary alkanes to examine the ability of our newly employed model to quantify the thermodiffusive behavior of the mixture components.

\subsection{Introduction}

In most of the applications, mixtures with scientific or technological interest are generally multicomponent. Owing to their relative simplicity, binary systems have been studied more extensively in the field of thermodiffusion. The development of experimental practices and the extraction of accurate data on diffusion and thermodiffusion coefficients are extremely 
vital for advancing the theory of transport properties of multi-component mixtures and its applications to natural and technological processes. It is only during the last decade that the focus has begun to shift toward ternary mixtures, which may be viewed as the simplest truly multicomponent mixtures [14, 72, 77, 82]. The complexity of Soret experiments has increased considerably with the increase in the number of components. Technical challenges and experimental errors can creep in due to the several external factors including earth gravity in ground conditions which causes convective instabilities in the cavity domain, mechanical vibrations, the lack of a detailed and elaborated processing of the experiment, and the handling of the mixture constituents. Performing experiments under microgravity conditions was one possible solution to produce data in convection-free conditions with least perturbation effects of gravity, while taking into account the impact of g-jitter vibration [14, 15, 82]. In the framework of the DCMIX (Diffusion Coefficient measurements in ternary mixtures) project, the microgravity platform has offered the benchmarking of ground measurements with the benefit of convection free conditions. Subsequently, more work on thermodiffusion measurements has increased with the aim to contribute to the experimental database in multicomponent mixtures. The most recent progress has been the establishment of the first benchmark on the ternary mixtures tetrahydronaphthalene (THN) + isobutylbenzene (IBB) $+\mathrm{n}$-dodecane $\left(\mathrm{nC}_{12}\right)$ at $(0.8,0.1,0.1)$ mass fractions [82]. The goal of this benchmark is to incorporate the results of Soret coefficients measured by six independent teams utilizing different experimental techniques in microgravity and ground conditions. Furthermore, Srinivasan and Saghir conducted experimental investigations on the Soret effect of ternary hydrocarbon mixtures composed of methane $\left(\mathrm{nC}_{1}\right)$, n-butane $\left(\mathrm{nC}_{4}\right)$, and n-dodecane $\left(\mathrm{nC}_{12}\right)$ for five different molar compositions. The experimental measurements were done at 333.15 $\mathrm{K}$ and $35 \mathrm{MPa}$ in a microgravity environment on board the satellite FOTON-M [15]. In their work, they justified the physics of separation direction and magnitude of mixture components (light, heavy, and intermediate).

Despite the recent progress, experimental values on ternary mixtures are still scarce and limited. Tests conducted in space cannot be considered fully reliable: they still face some technical issues concerning design imperfections of cells on board, perturbations in the component separation, and sensitivity of data post-processing [14, 82]. A detailed review of the experimental techniques of thermodiffusion can be found in Ref. [14]. This shortfall of multicomponent data has impacted the improvement of the current numerical models and 
the formulation of a clear unified thermodiffusion theory that can emulate the separation physics with high accuracy.

Nevertheless, some progress has been made in the theoretical modeling of thermodiffusion [9, 53, 83-86]. However, a number of open questions still exist regarding the limitations of the current theoretical approaches when applied to describe thermodiffusion in multicomponent petroleum fluids at typical reservoir conditions. As discussed in the review of Eslamian and Saghir [85], most of these models are based on non-equilibrium thermodynamic, kinetic equations, or mechanical expressions. The major drawback of such models is predicting the accuracy of thermodynamics properties using the equations of state. A few attempts have been done on the development of theoretical approaches to predict thermal diffusion factors in multi-component mixtures [85, 86]. However, there is still discrepancy between the models: they fail to account for the complex composition dependence and relative properties contributions to the Soret coefficient, which is due to the cross-interactions that happen at the molecular level [9].

At present, very limited work has been done to study thermodiffusion in ternary hydrocarbon mixtures using MD. Galliero et al. [87] conducted boundary driven non-equilibrium molecular dynamics (BD-NEMD) simulations to examine the thermodiffusion coefficient of $\left(\mathrm{nC}_{1}-\mathrm{nC}_{4}-\mathrm{nC}_{12}\right)$ ternary mixtures at $333.15 \mathrm{~K}$ and $35 \mathrm{MPa}$ for five different molar compositions using different fluid models: Lennard-Jones (LJ) and Lennard-Jones Chain (LJC) fluid models. Later, Artola and Rousseau [54] did extensive MD simulations on ternary isotopic mixtures using the LJ sphere model. They compared their NEMD results with the data obtained using the theoretical approach based on the extended Prigogine model, which showed a remarkable agreement only for the isotopic ternary mixtures.

In this work, the eHEX NEMD algorithm along with the augmented TraPPE-UA is proposed to study thermodiffusion in n-alkane ternary mixtures. Our employed methodology is already validated in Chapter 4 for the binary mixture $\left(\mathrm{nC}_{5}-\mathrm{nC}_{10}\right)$ at three different compositions. In order to test the feasibility of this approach in ternary mixtures, the thermodiffusion factor of the $\mathrm{nC}_{1}-\mathrm{nC}_{4}-\mathrm{nC}_{12}$ mixture for five different compositions was calculated at $333.15 \mathrm{~K}$ and $35 \mathrm{MPa}$ and validated with respect to the experimental data of Srinivasan and Saghir16 and compared to the MD work of Galliero et al. [87] In the attempt to understand the 
physics behind the separation response in each mixture, speculative explanations are given based on an inherent assumption that thermal diffusion is an elementary process where two molecules of different species swap positions. The possibility of a cross-interaction between the 2 molecules is influenced by many factors some of which have competing effects. In section 4.3 , the computational details of EMD and NEMD methodology are described. Section 5.3 presents the validity of numerical results pertaining thermodynamics properties of liquid mixtures (density, diffusion coefficient) followed by an analysis of the thermodiffusion separation ratio results obtained for the different cases. Finally, the summary of our research study and the pertinent conclusions are drawn in section 5.4 .

\subsection{Methodology}

\subsubsection{EMD}

To assess the performance of the force field model in ternary mixtures and ensure equilibrium state, EMD simulations were conducted to obtain thermodynamics properties such as the system density and self-diffusion coefficient of each component in the mixture. Simulations were carried out at $333.15 \mathrm{~K}$ and $35 \mathrm{MPa}$, and Periodic Boundary Conditions (PBC) were applied for all systems. A total of 2000 molecules were included in the systems. In order to avoid highly overlapped atoms, the system configuration was initially brought to a local potential energy minimum by performing an energy minimization. Next, an annealing procedure was used in order to accelerate the equilibration and avoid trapping in a local minimum of energy: the system was initially heated up to $700 \mathrm{~K}$ and then cooled down back to 333.15 K. Next, the NPT ensemble with a Nose Hoover thermostat and barostat using the time constants of 0.1 and 1 ps, respectively, was employed in order to determine the equilibrium ternary system densities at a target pressure of $35 \mathrm{MPa}$ for the 5 mixture compositions. Long-range tail corrections to the energy and pressure were included. The equations of motion were integrated using the velocity Verlet algorithm, and a time step of 1 fs was used in all simulation runs. To bring the system to an equilibrium state and ensure energy conservation, EMD simulations were performed in the NVT ensemble using the Nose Hoover thermostat at the previously determined equilibrium density corresponding to a pressure of $35 \mathrm{MPa}$. After running NVT for $2 \mathrm{~ns}$, the average system energy was calculated using the NVE ensemble. The energy of the last configuration of the NVT simulation 
was adjusted by carrying out an additional NVE equilibration run for 5 ns. This procedure allowed us to achieve an average equilibrium temperature of $333.15 \pm 1 \mathrm{~K}$. The final configuration obtained was used as an initial configuration in the simulations for determining D, which is also performed again in the NVE ensemble with an additional run time of 5 ns. The self-diffusion coefficient $\left(D_{i, s e l f}\right)$ of each constituent of type $\mathrm{i}$ in the ternary mixture $\left(\mathrm{nC}_{1}-\mathrm{nC}_{4}-\mathrm{nC}_{12}\right)$ was computed to explain the effect of the diffusion rate with the system and validate the performance of the force field model applied on ternary liquid mixtures (see Eq. 4.7). The mean square displacement (MSD), which measures the average distance traveled by a given particle in a system, is used to describe the behavior and the atomic diffusivity of the alkane molecules.

\subsubsection{BD-NEMD}

The system size and strength of the heat flux were considered as two important parameters to achieve good computational results and local equilibrium, as defined by the validity of thermodynamic equations [8]. The criteria of the validity of local equilibrium from NEMD, clearly formulated in the work of Kjelstrup et al. [42], were checked in order to verify the reliability of our computational results under extreme temperature gradients.

In order to ensure a good statistical precision, all NEMD ternary systems contained 2000 molecules (given composition). With the previously obtained densities, ternary mixture configurations were equilibrated again using NVT and NVE and a time step of $1 \mathrm{fs}$. The system region was modified for BD-NEMD simulations. The box dimensions were rescaled such that its length in the $z$ direction $\left(L_{z}\right)$ is twice its length in $x\left(L_{x}\right)$ and $y\left(L_{y}\right)$ directions, while maintaining the same equilibrated system density. A unidirectional heat flux was imposed on the system along the z-axis. The two thermostat regions were centered at the points $z= \pm L_{z} / 4$ (see Fig. 4.1). During each time step, a fixed amount of heat is taken from the cold zone and added to hot zone. In order to capture the spatial variation of the temperature and composition, the simulation box was divided in the $\mathrm{z}$ direction into 32 bins of equal size. The number of molecules inside each slab (around 62 molecules) was considered large enough for it to behave as a thermodynamic system [42. Each BD-NEMD simulation (given

composition) was at least $48 \times 10^{6}$ time steps long (after an equilibrium simulation of $5 \times 10^{6}$ time steps) with a larger time step of 2 fs for which the total system energy exhibited a 
minor drift (less than $0.3 \%$ ). The first $2.5 \times 10^{6}$ time steps of simulation were discarded from the average calculations to avoid transient effects. Local temperature and density fraction were computed at each 500 time steps, and averages of 20 samples were dumped every 10 000 time steps to a file for later analysis. Additionally, accumulated averages of the system overall energy and temperature and the local mole fraction and temperature for each layer were computed [8]. The corresponding gradients were also calculated from a linear fit of the local quantities. The hot and cold regions (4 slabs each) as well as two neighbor slabs have been discarded from the temperature gradient measurement because of non-linear behavior in these layers. The statistical errors were estimated from the standard deviation from the mean.

For better physical insight into the separation process, both thermodiffusion factor $\alpha_{T, i}$ and the so-called separation ratio $k_{T, i}^{\prime}$ corresponding to the Soret quantity were evaluated from the proportionality coefficient relating the mole fraction gradient to thermal gradient for each block, according to the following equations:

$$
\begin{aligned}
k_{T, i}^{\prime} & =-\frac{\nabla x_{i}}{\nabla T} \\
\alpha_{T, i} & =-T \frac{\nabla x_{i}}{x_{i}\left(1-x_{i}\right) \nabla T}
\end{aligned}
$$

where $\nabla T$ is the temperature gradient and $\nabla x_{i}$ is the component type i mole fraction gradient. The thermodiffusion separation ratio was computed as a running average over the time blocks, and the statistical error was estimated from the standard deviation from the mean.

\subsection{Results and Discussion}

\subsubsection{Validation of mixture density and self-diffusion coefficient}

The employed potential model was validated by comparing simulation densities for all mixtures with the experimental data obtained from NIST [88], as shown in Table 5.1. The numerical values provide a reasonable verification of the potential model parameters by revealing a maximum error of $1.64 \%$ (obtained for mixture 4 ) with respect to the experimental 
data. Thus, our UA force field is a good candidate for accurately modeling the interactions

Table 5.1: Densities of $\mathrm{nC}_{1}-\mathrm{nC}_{4}-\mathrm{nC}_{12}$ mixture for different molar composition at $333.15 \mathrm{~K}$ and $35 \mathrm{MPa}$. The $\%$ error $\Delta$ of the numerical density of this work was computed with respect to experimental data.

\begin{tabular}{lcccccc}
\hline \hline & \multicolumn{3}{c}{ Mole fraction } & \multicolumn{2}{c}{$\begin{array}{c}\text { Size of simulation } \\
\text { box }\left(\mathrm{A}^{3}\right)\end{array}$} & \multicolumn{2}{c}{ Density $\left(\mathrm{kg} / \mathrm{m}^{3}\right)$} \\
\cline { 2 - 3 } \cline { 7 - 7 } Mixture & $\mathrm{nC}_{1}$ & $\mathrm{nC}_{4}$ & $\mathrm{nC}_{12}$ & & $\mathrm{NIST}$ & MD Work \\
\hline 1 & 0.2 & 0.1 & 0.7 & 604180.21 & 744.32 & $736.16(1.10)$ \\
2 & 0.2 & 0.2 & 0.6 & 549809.33 & 734.28 & $728.21(0.83)$ \\
3 & 0.2 & 0.4 & 0.4 & 457988.35 & 703.09 & $696.56(0.93)$ \\
4 & 0.2 & 0.5 & 0.3 & 419034.49 & 679.64 & $668.52(1.64)$ \\
5 & 0.2 & 0.6 & 0.2 & 379212.89 & 648.72 & $643.44(0.81)$ \\
\hline \hline
\end{tabular}

and transport properties of the ternary n-alkane mixtures at high pressure and temperature, for the different molar compositions.

Another benchmark for model validation is the evaluation of the dispersed and aggregated state of the ternary system (interactions between molecules) for different molecular compositions. The justification is done by calculating the self-diffusion coefficients of the system components. Figure 5.1 illustrates the MSD of $\mathrm{nC}_{1}, \mathrm{nC}_{4}$, and $\mathrm{nC}_{12}$ evolution with respect to the EMD simulation time for mixtures 2, 3, 4, and 5 at $333.15 \mathrm{~K}$ and $35 \mathrm{MPa}$. A great linear trend of MSD was observed for all the species.

The self-diffusion coefficients were calculated from the slope of MSD lines and presented in Table 5.2. In comparison with the results in the literature for pure and binary n-alkanes, the governing self-diffusion coefficients lie in the same magnitudes at identical temperature and pressure conditions. The overall increase in self-diffusion coefficients of each n-alkane constituent $\left(\mathrm{nC}_{1}, \mathrm{nC}_{4}\right.$, and $\left.\mathrm{nC}_{12}\right)$ with the increase in $\mathrm{nC}_{4}$ mole fraction proved the effect of system density, species relative weight, and activation energy on the molecular displacement. This behavior is expected and explained by the increase in the system molecular loading which intensifies the collisions between particles, thereby raising the energy barriers for 
diffusion. In other words, the accuracy of the calculated results in Table 5.2 is acceptable and the existing force field model accurately describes the molecular interactions in ternary n-alkane mixtures.

Table 5.2: Self-diffusion coefficients $(D)$ of $\mathrm{nC}_{1}, \mathrm{nC}_{4}$ and $\mathrm{nC}_{12}$ for the different ternary mixture molar compositions.

\begin{tabular}{cccc}
\hline \hline \multirow{2}{*}{$\mathrm{nC}_{4}$ mole fraction } & \multicolumn{3}{c}{$D \times 10^{-9}\left(\mathrm{~m}^{2} / \mathrm{s}\right)$} \\
\cline { 2 - 4 } & $\mathrm{nC}_{1}$ & $\mathrm{nC}_{4}$ & $\mathrm{nC}_{12}$ \\
\hline 0.1 & 3.87 & 2.03 & 1.08 \\
0.2 & 4.29 & 2.52 & 1.32 \\
0.4 & 4.48 & 3.04 & 1.43 \\
0.5 & 5.24 & 3.40 & 1.82 \\
0.6 & 14.96 & 8.98 & 5.05 \\
\hline \hline
\end{tabular}

\section{Linearity and convergence of non-equilibrium MD process}

Local equilibrium and system convergence were examined by tracking the variation of temperature, composition, and energy (potential and kinetic) in each slab over simulation time steps. In order to have a similar evaluation scaling factor, normalized values of all time evolution variables were considered with respect to steady state mean values and plotted against simulation time, according to the following formula:

$$
y^{*}\left(t_{i}\right)=\frac{y\left(t_{i}\right)}{y_{\infty}}
$$

where $y\left(t_{i}\right)$ indicates any variable at time $t_{i}$ and $y_{\infty}$ is the estimated mean value of the variable. Figure 5.1 shows the normalized kinetic (Ke), potential (Pe), and total energy in three different regions of the simulation box (hot, cold, and middle). For all compositions, stationary state was attained with minimal fluctuations at the end of the production run. Moreover, it is noticed that as the mixture density increases, the convergence time increases for Pe, while it decreases for Ke. The fluctuations in Ke at the beginning of the simulation runs for low mixture density (mixture 5 to 1 ) is due to the increased amount of lighter molecules that require lower activation energy. On the other hand, as mixture density increases the intermolecular interactions between molecules increase due to heavier component collisions, 
which develops Pe instabilities.

The continuity profile of steady state local density, temperature, and concentration was verified for each $\mathrm{nC}_{1}-\mathrm{nC}_{4}-\mathrm{nC}_{12}$ mixture composition. The linear trend of density and temperature profiles across the simulation box for mixtures 25 indicates that the system behavior, despite strong gradients, is still in the domain of the linear response and behaves like a liquid [43. The high pressure of $35 \mathrm{MPa}$ ensured that methane remained in the liquid state in the mixture. In order to check for concentration linearity, the mole fraction profiles for each component were evaluated for all compositions. Figure 5.2 verifies the linear trend and direction of concentration slopes attained in mixtures 2,3 , and 4 . The dotted lines correspond to a linear fit of the data. To support this assumption, the criteria of local equilibrium from NEMD were checked [42]. Each layer contained around 62-63 molecules. The average fluctuation estimates of local concentration and temperature were around 4\%-9\% and 0.3\%-0.5\%, respectively, in each slab. The relative change in temperature and density across the slab in the direction of diffusion was smaller than one. The linearity condition of both gradients was also evaluated through the R2, which achieved a value higher than 0.97 and 0.99 for composition and temperature, respectively. The observed local convergence demonstrates that n-alkane ternary systems are in local equilibrium state prior to data post-processing. 

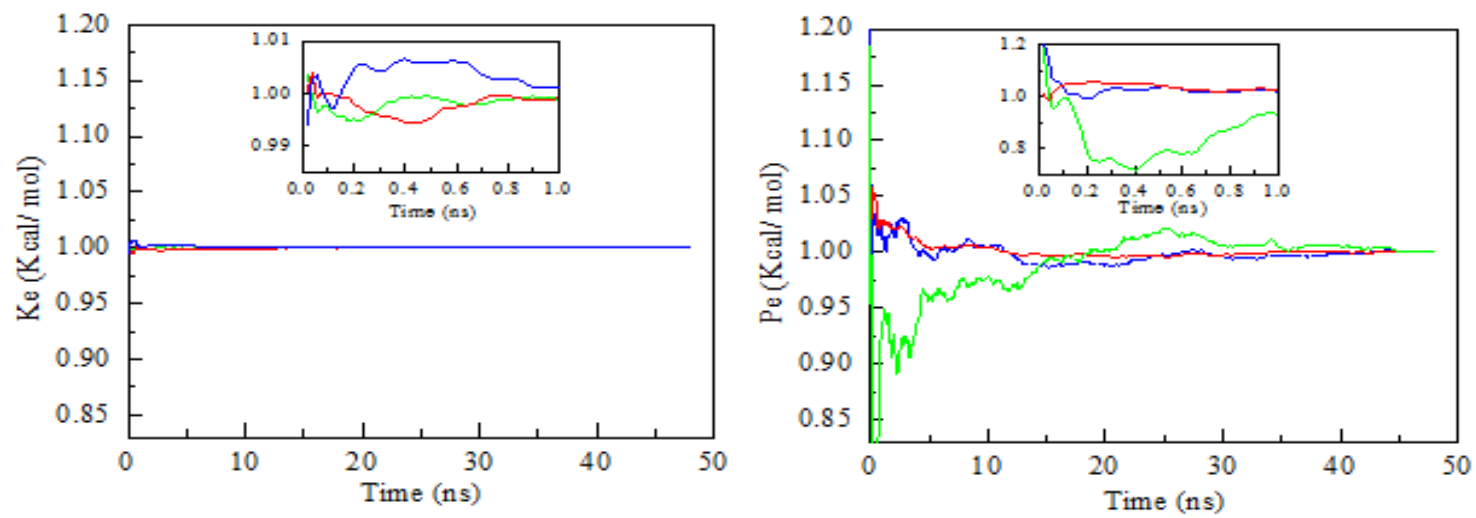

(a)
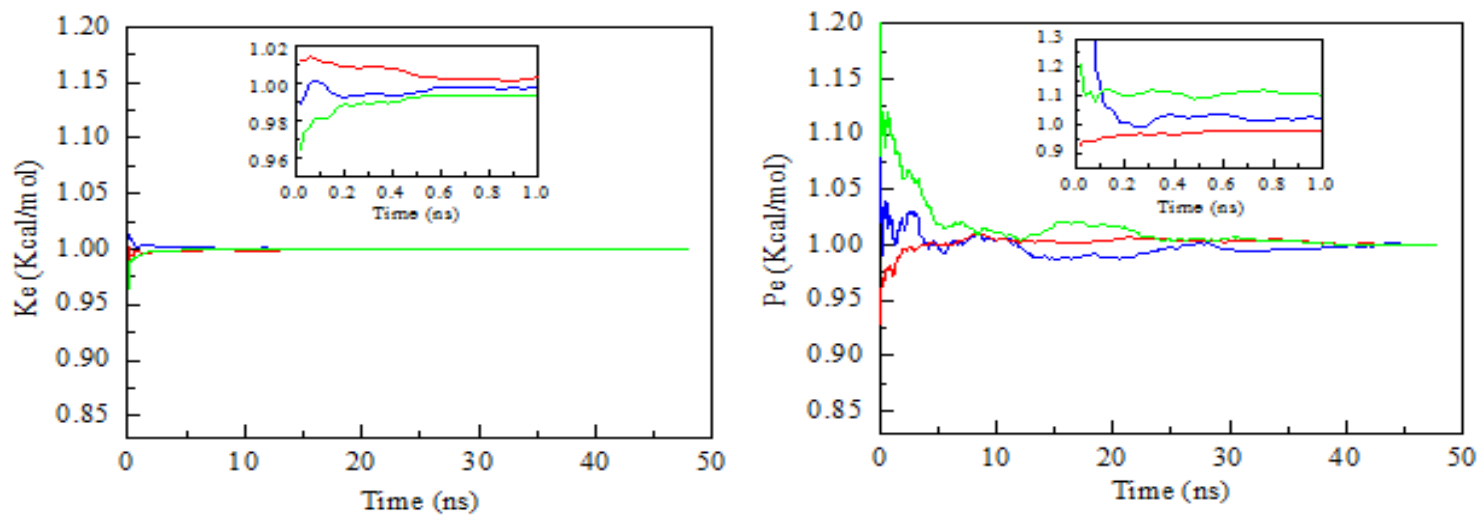

(b)
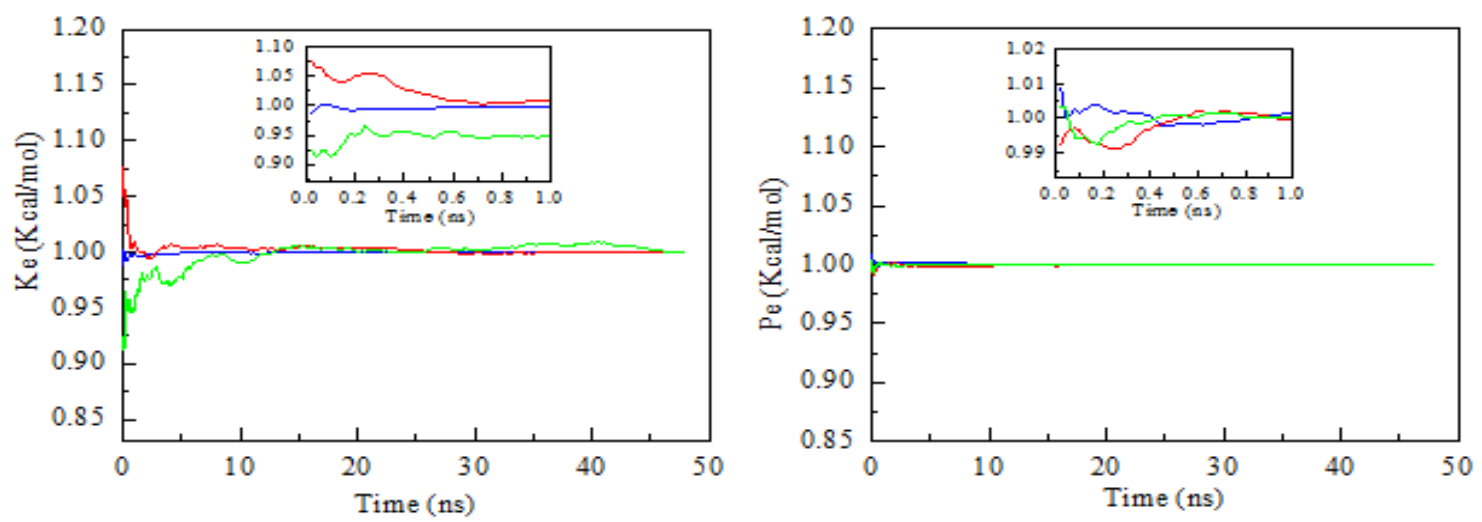

(c)

Figure 5.1: Normalized local kinetic energy (left) and potential energy (right) variation with NEMD simulation time in hot (red), cold (blue), and middle (green) slab regions and represented for (a) mixture $2\left(\mathrm{xC}_{4}=0.2\right)$, (b) mixture $3\left(\mathrm{xC}_{4}=0.4\right)$, and (c) mixture $5\left(\mathrm{xC}_{4}\right.$ $=0.6)$. 

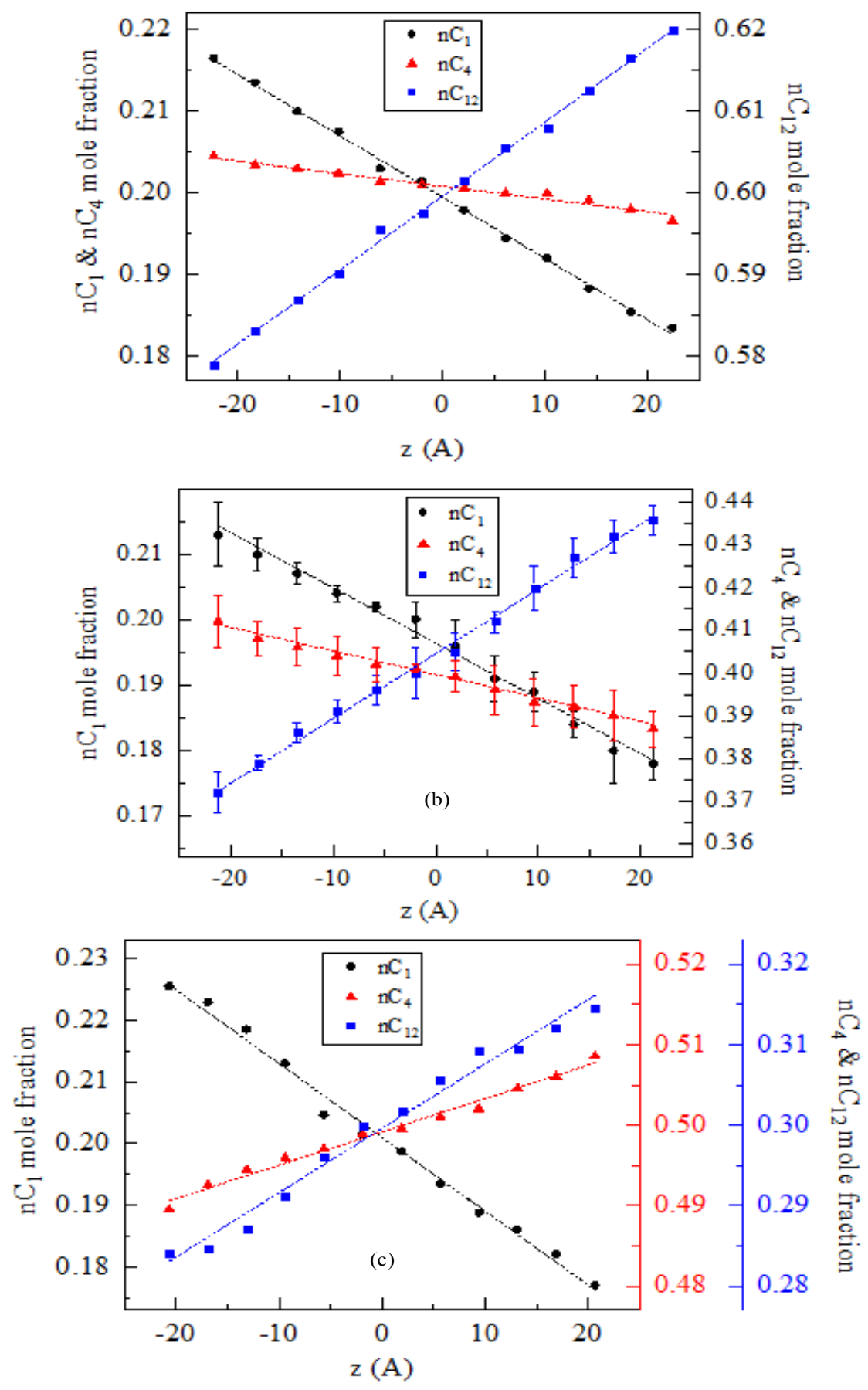

Figure 5.2: Mole fraction profile of $\mathrm{nC} 1$ (black dots), $\mathrm{nC} 4$ (red triangles), and $\mathrm{nC} 12$ (blue squares) for three different molar compositions (a) mixture $2\left(\mathrm{xC}_{4}=0.2\right)$, (b) mixture $3\left(\mathrm{xC}_{4}\right.$ $=0.4)$, and $(\mathrm{c})$ mixture $4\left(\mathrm{xC}_{4}=0.5\right)$. The error bars which represent the standard deviation of the mean are only shown for mixture 3 
When dealing with large multicomponent systems, it is important to look at the time evolution of each components concentration gradients. Figure 5.3 represents the running averages of the normalized mole fraction gradients of each component in mixtures 2 - 5 , evaluated since the onset of the production run. High oscillations were detected at the beginning of NEMD simulations, which eventually attenuate at the end of the run as shown in Figure 5.3. As expected, the time needed for species mole fraction gradient to reach a plateau or local equilibrium was significantly higher than that of the temperature gradient due to higher instabilities in particles movement and collisions (check Ke in Figure 5.1 for temperature gradient time evolution and Figure 5.3 for concentration gradient time evolution). Note that the errors (standard deviation of the mean) in local composition and temperature were taken into account to compute profiles and their uncertainties. The standard error of the thermodiffusion separation ratio was computed with composition and temperature profile uncertainties using standard error propagation methods [74].
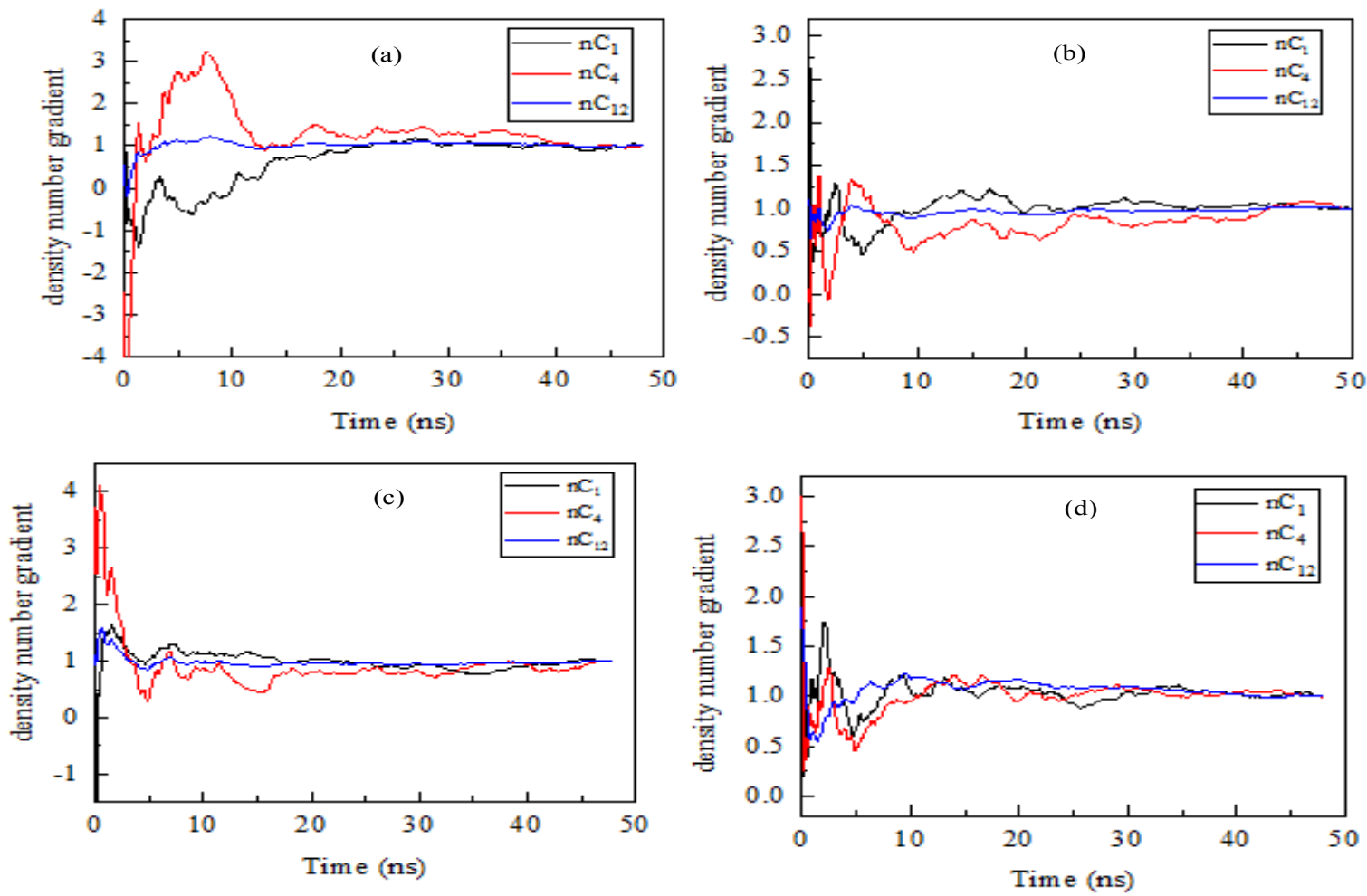

Figure 5.3: Normalized concentration (density number) gradient variation with NEMD simulation time for each component in (a) mixture $2\left(\mathrm{xC}_{4}=0.2\right)$, (b) mixture $3\left(\mathrm{xC}_{4}=0.4\right)$, (c) mixture $4\left(\mathrm{xC}_{4}=0.5\right)$, and $(\mathrm{d})$ mixture $5\left(\mathrm{xC}_{4}=0.6\right.$. 


\subsubsection{Thermodiffusion Factor}

The main aspect of understanding the thermodiffusion process is to be able to identify the direction (sign) and strength (magnitude) of separation of the components in a mixture when a thermal gradient is applied. According to the previous findings [9, 15, 54] the particular interactions leading to a certain thermodiffusion separation direction and magnitude are dependent on the combination of different factors such as the activation energy, size, similarity, and mole fraction of system components. For instance, species with a relatively large mole fraction would imply that the latter dictates the segregation process by being the dominant creator of holes as well as by being available in large quantities to fill a hole. Additionally, large molecules (higher density) involve higher activation energy and lower mobility, which renders their diffusion even slower. The opposite applies for smaller molecules. Note that in mixtures of linear n-alkanes composed of flexible and chemically akin molecules (nonassociated mixtures), one can expect an isotope-like behavior of the system [9, 14, 54].

Keeping in mind the elements responsible of forming a particular molecular interaction, the trends of the thermodiffusion factor of $\mathrm{nC}_{1}, \mathrm{nC}_{4}$ and $\mathrm{nC}_{12}$ in these mixtures can be explained based on the inherent assumption of the probability of a molecule to occupy or create a hole formed by other types of species. In other words, the separation process can be explained via three probable interactions: $\mathrm{nC}_{1}-\mathrm{nC}_{4}, \mathrm{nC}_{1}-\mathrm{nC}_{12}$, and $\mathrm{nC}_{4}-\mathrm{nC}_{12}$. These interactions are largely impacted by the mole fraction, similarity, and the relative difference of mass and activation energy of the two molecules involved in creating and occupying a hole.

The thermal diffusion of each component for the different mixture compositions was carefully examined. The experimental and numerical values obtained by this work and the MD model of Galliero et al. [1] are shown in Figure 5.4. Data with the corresponding relative errors are also listed in Table 5.3 . 
Table 5.3: Numerical values of thermodiffusion factor computed for each component using this work MD algorithm and other models of Galliero [1] for all the molar compositions. The values in brackets represent the percentage error with respect to the experimental values.

\begin{tabular}{|c|c|c|c|c|c|}
\hline \multirow{2}{*}{$\begin{array}{c}\mathrm{nC}_{4} \\
\text { mole fraction }\end{array}$} & \multicolumn{5}{|c|}{$\mathrm{nC}_{4}$ thermodiffusion factor $\alpha_{C_{4}}$} \\
\hline & Exp. & This work & $\mathrm{LJC} / \mathrm{LB}$ & $\mathrm{LJC} / \mathrm{KG}$ & LJC/WH \\
\hline 0.10 & -0.22 & $-0.44(38.64)$ & $-0.41(86.87)$ & $-0.42(91.92)$ & $-0.47(112.12)$ \\
\hline 0.20 & -0.25 & $-0.24(2.55)$ & $-0.42(67.5)$ & $-0.39(55.00)$ & $-0.38(52.50)$ \\
\hline 0.40 & -0.5 & $-0.49(38.92)$ & $-0.37(25.83)$ & $-0.48(4.25)$ & $-0.51(1.67)$ \\
\hline 0.50 & 0.55 & $0.32(41.85)$ & $-0.31(156.73)$ & $-0.29(153.09)$ & $-0.31(156.00)$ \\
\hline 0.60 & 0.82 & $0.74(10.28)$ & $-0.10(112.70)$ & $-0.13(115.24)$ & $-0.18(121.85)$ \\
\hline \multirow{2}{*}{$\begin{array}{c}\mathrm{nC}_{4} \\
\text { mole fraction }\end{array}$} & \multicolumn{5}{|c|}{$\mathrm{nC}_{1}$ thermodiffusion factor $\alpha_{C_{1}}$} \\
\hline & Exp. & This work & $\mathrm{LJC} / \mathrm{LB}$ & $\mathrm{LJC} / \mathrm{KG}$ & LJC/WH \\
\hline 0.10 & -1.53 & $-1.31(14.26)$ & $-1.03(32.60)$ & $-1.13(26.06)$ & $-1.12(26.88)$ \\
\hline 0.20 & -1.55 & $-1.21(22.09)$ & $-1.02(34.27)$ & $-1.07(31.05)$ & $-1.14(26.21)$ \\
\hline 0.40 & -1.20 & $-1.12(2.83)$ & $-0.95(20.83)$ & $-1.01(16.09)$ & $-0.99(17.19)$ \\
\hline 0.50 & -1.76 & $-1.44(18.37)$ & $-0.89(49.22)$ & $-0.99(43.89)$ & $-1.08(38.92)$ \\
\hline 0.60 & -1.71 & $-1.71(0.15)$ & $-0.91(46.64)$ & $-0.99(41.89)$ & $-0.97(43.35)$ \\
\hline \multirow{2}{*}{$\begin{array}{l}\mathrm{nC}_{4} \\
\text { mole fraction }\end{array}$} & \multicolumn{5}{|c|}{$\mathrm{nC}_{12}$ thermodiffusion factor $\alpha_{C_{12}}$} \\
\hline & Exp. & This work & $\mathrm{LJC} / \mathrm{LB}$ & $\mathrm{LJC} / \mathrm{KG}$ & LJC/WH \\
\hline 0.10 & 1.26 & $1.13(10.30)$ & $2.24(23.66)$ & $2.43(17.23)$ & $2.46(16.48)$ \\
\hline 0.20 & 1.20 & $1.44(19.73)$ & $1.44(20.14)$ & $1.46(19.10)$ & $1.53(15.28)$ \\
\hline 0.40 & 1.30 & $1.24(4.62)$ & $1.00(22.76)$ & $1.15(11.54)$ & $1.17(9.94)$ \\
\hline 0.50 & 0.68 & $0.61(9.85)$ & $0.88(54.76)$ & $0.92(61.76)$ & $1.00(74.37)$ \\
\hline 0.60 & 0.47 & $0.40(15.53)$ & $0.71(127.39)$ & $0.79(151.33)$ & $0.83(163.30)$ \\
\hline
\end{tabular}



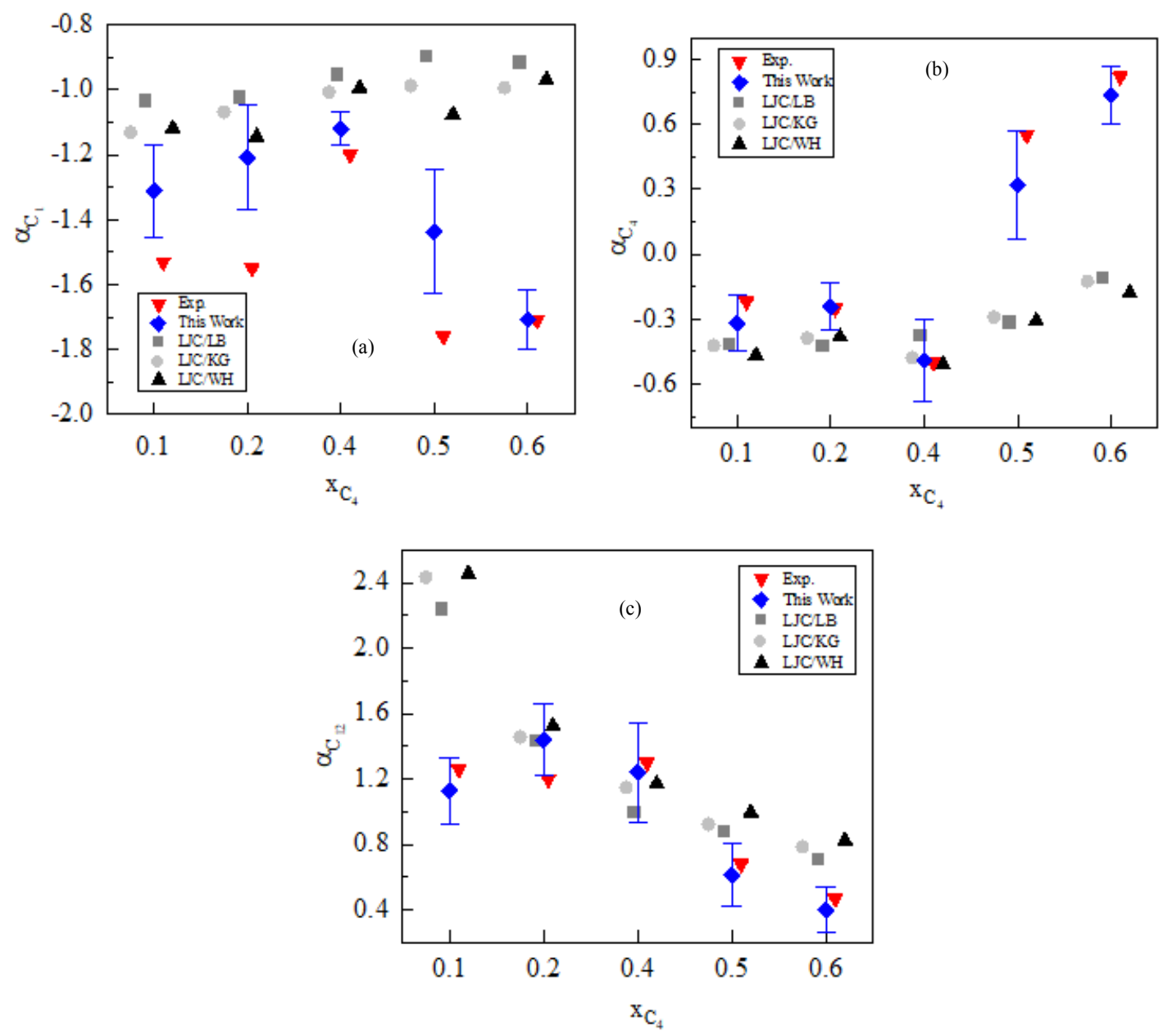

Figure 5.4: Comparison of the thermodiffusion factor for each component (a) $\mathrm{nC}_{1}$, (b) $\mathrm{nC}_{4}$, and $(c) \mathrm{nC}_{12}$ in the ternary mixture for all the five molar compositions. Values correspond to the experimental other numerical values in the literature and this work MD results. 
It is clear that there is good qualitative agreement between the experimental data and the MD model presented in this work, which presents the least deviation compared to the Galliero et al. MD model [1]. Specifically, the MD framework proposed in this work accurately predicts the change in the direction of separation for $\mathrm{nC}_{4}$ as its concentration in the mixture increases, while the Galliero et al. [1] model fails to predict the direction of the intermediate component $\left(\mathrm{nC}_{4}\right)$ for mixtures 3 and 4 . The large deviations of Galliero et al. [1] could probably be attributed to the fact that a detailed force field model was not used to produce the atomistic interactions. Also their model does not correct the energy losses in the original HEX algorithm.

Based on the findings in all the simulated mixtures, the heavier species $\left(\mathrm{nC}_{12}\right)$ always tend to enrich at the cold side and lighter species $\left(\mathrm{nC}_{1}\right)$ tend to go preferentially to the hot side. This behavior is in agreement with the thermophobicity concept of Hartmann and co-workers [89] and can be explained as follows: In the case of $\mathrm{nC}_{12}$, in all the mixtures, it mostly appears in $\mathrm{nC}_{1}-\mathrm{nC}_{12}$ or $\mathrm{nC}_{4}-\mathrm{nC}_{12}$ interactions. Since in either case it is the denser component with the highest activation energy, it always moves to the cold side; i.e., it has a positive thermodiffusion factor. On the other hand, $\mathrm{nC}_{1}$ is largely involved in $\mathrm{nC}_{1}-\mathrm{nC}_{12}$ or $\mathrm{nC}_{1}-\mathrm{nC}_{4}$ interactions and is the component with the lowest activation energy in these interactions. Hence, it always segregates to the hot side; i.e., it has a negative thermodiffusion factor. On the other hand, in ternary mixtures 1, 2, and 3, the component with an intermediate density, $\mathrm{nC}_{4}$, has a positive slope segregating to the hot reservoir. In mixtures 4 and $5, \mathrm{nC}_{4}$ separates to the cold reservoir (see Figure 5.4). The sign change of thermodiffusion with the molar composition of $\mathrm{nC}_{4}$ implies that its relative concentration with respect to $\mathrm{nC}_{1}$ and $\mathrm{nC}_{12}$ in the mixture directly influences its direction of segregation.

For a better understanding of the physical insight into the magnitude of thermodiffusion, the results of the separation ratio $k_{T, i}^{\prime}$ are summarized in Table 5.4 .

In the first 3 mixtures, $\mathrm{nC}_{12}$ is the dominant species and exhibits the highest proportion of separation relative to $\mathrm{nC}_{1}$ and $\mathrm{nC}_{4}$. This is due to the considerable amount of $\mathrm{nC}_{12}$ molecules contributing in more possible interactions of $\mathrm{nC}_{1}-\mathrm{nC}_{12}$ and $\mathrm{nC}_{4}-\mathrm{nC}_{12}$. The fewer $\mathrm{nC}_{1}-\mathrm{nC}_{4}$ interactions resulted in a net motion of $\mathrm{nC}_{4}$ toward the hot side as the number of these interactions is too small to overcome $\mathrm{nC}_{4}-\mathrm{nC}_{12}$ interactions. 
Table 5.4: Experimental and this work MD results of thermodiffusion separation ratio $k_{T, i}^{\prime}$ for each component at every mixture mole fraction.

\begin{tabular}{|c|c|c|c|c|c|c|}
\hline \multirow{3}{*}{$\begin{array}{l}\mathrm{nC}_{4} \\
\text { mole } \\
\text { fraction }\end{array}$} & \multicolumn{6}{|c|}{ Separation ratio $k_{T, i}^{\prime} * 1000$} \\
\hline & \multicolumn{2}{|c|}{$\mathrm{nC}_{1}$} & \multicolumn{2}{|c|}{$\mathrm{nC}_{4}$} & \multicolumn{2}{|c|}{$\mathrm{nC}_{12}$} \\
\hline & Exp. & $\begin{array}{l}\text { This } \\
\text { work }\end{array}$ & Exp. & $\begin{array}{l}\text { This } \\
\text { work }\end{array}$ & Exp. & $\begin{array}{l}\text { This } \\
\text { work }\end{array}$ \\
\hline 0.1 & -0.73 & -0.63 & -0.06 & -0.08 & 0.79 & 0.71 \\
\hline 0.2 & -0.74 & -0.58 & -0.12 & -0.12 & 0.86 & 1.04 \\
\hline 0.4 & -0.58 & -0.56 & -0.36 & -0.22 & 0.94 & 0.89 \\
\hline 0.5 & -0.85 & -0.69 & 0.41 & 0.24 & 0.43 & 0.39 \\
\hline 0.6 & -0.82 & -0.82 & 0.59 & 0.53 & 0.23 & 0.19 \\
\hline
\end{tabular}

As we move from mixtures 1 to 5 , the absolute value of the $\mathrm{nC}_{4}$ separation ratio continues to rise until it reaches a maximum value of 0.53 . This behavior is speculated by the relatively intermediate density of $\mathrm{nC}_{4}$ and the gradually increasing mole fraction. Put differently, as $\mathrm{nC}_{4}$ becomes the dominant species (as in the case of the final two mixtures), there is an increase in $\mathrm{nC}_{4}-\mathrm{nC}_{1}$ interactions and a reduction in $\mathrm{nC}_{4}-\mathrm{nC}_{12}$ interactions. As a result, there is a net movement of $\mathrm{nC}_{4}$ to the cold side. The reason that the separation strength is higher toward the cold region is hypothesized by the fact that $\mathrm{nC}_{4}$ molecules become more mobile as the mixture density decreases.

In the case of $\mathrm{nC}_{1}$, the lowest thermodiffusive separation is observed in mixture 2 (0.58 ) and the highest in mixture 5 (-0.82). In fact, $\mathrm{nC}_{1}-\mathrm{nC}_{4}$ interactions in mixture 5 are stronger than $\mathrm{nC}_{1}-\mathrm{nC}_{12}$ interactions in mixture 2 , for the same molar composition ratio of the 2 species involved. This behavior can be explained by the decrease of system molecular loading (lower density) in mixture 5, which diminishes the collisions between particles and consequently causes a drop in energy barriers in the way of diffusion. Finally, the effect of mixture composition, cross-interactions, mobility, and similarity features of ternary mixture components highly influence the sign and magnitude of the thermodiffusion factor.

In summary, the proposed MD approach can precisely reproduce the molecular behavior of the different mixture species when subject to a thermal field, while conserving the system 
energy with minor numerical instabilities. In other words, the thermophobicity concept along with the effect of relative mixture composition on the magnitude and sign of the thermodiffusion factor of each component in the ternary mixture is demonstrated fairly accurately in our NEMD results.

\subsection{Conclusion}

The performance of Boundary Driven Non-Equilibrium Molecular Dynamics (BD-NEMD) using the enhanced heat exchange (eHEX) algorithm was studied in order to test its ability to accurately predict thermodiffusion in $\mathrm{nC}_{1}-\mathrm{nC}_{4}-\mathrm{nC}_{12}$ ternary mixtures for five different molar compositions. The eHEX algorithm is an extended version of the HEX algorithm, developed by Hafskjold and co-workers with an improved energy conservation. It was applied along with the augmented TraPPE-UA force field. The thermodiffusion factor or separation ratio for all ternary mixture compositions was assessed at $333.15 \mathrm{~K}$ and $35 \mathrm{MPa}$ and compared to the experimental data as well as 3 other models from the literature [1]. The following key observations were drawn from the findings:

1. The computational precision of our enhanced algorithm along with the UA force field model manage to accurately describe the molecular interactions in ternary n-alkane mixtures and predict mixture densities and self-diffusion coefficients for each component. Moreover, our MD approach achieved local equilibrium with great convergence while significantly conserving the system energy with minor instabilities.

2. The continuity of the gradients (temperature, composition, and mixture density) emphasizes that the system behavior, despite the extreme gradients, is still in the domain of the linear response. The time evolution of both local energies and species concentration gradients converges with minimal computational error.

3. The model proposed in this work showed good agreement with experimental data and performed better than the other models proposed by Galliero et al. [84]. More specifically, our MD model accurately captures the change in the sign of $\mathrm{nC}_{4}$ separation as its concentration in the mixture increases, which was not possible in Ref. [84].

In conclusion, the MD framework proposed in this work can adequately represent the physics behind the thermodiffusive separation and we can explain the segregation based on 
the composition dependent interactions between the different species in the mixture. Given that our proposed model performed well for calculating the thermodiffusion factor of ternary n-alkane mixtures, the eHEX algorithm along with the augmented TraPPE-UA force field model serves as a highly promising framework and a low-cost alternative for experiments to study thermodiffusion in ternary mixtures. 


\section{Chapter 6}

\section{Molecular Dynamics Evaluation of Alumina Nanofluid Thermal Transport Performance}

This chapter has been reproduced, nearly exactly, from our submitted paper work to refer directly to Chapter 6 of this thesis. The submitted manuscript appears as in [90]: S. Antoun, M. Z. Saghir "Thermophysical Properties and Heat Transfer Enhancement of Alumina Nanofluid: An Improved Molecular Dynamics Study."

\subsection{Introduction}

The suspension of nanoparticles (NPs) in aqueous solutions, known as nanofluid, hold promise in many energy applications, including thermal management, energy storages, sustainable power harvesting for cooling/heating purposes and others, owing to unique thermophysical properties obtained by adding NPs into conventional fluids [20, 21]. Extensive research has been conducted to evaluate thermal enhancement, but despite the progress brought by experimental effort, real understanding of the nature of heat transfer enhancement mechanisms in nanofluids has yet to be achieved [6, 18. Many studies in the literature offer conflicting experimental results [4, 91 95]. Discrepancies stem from a multitude of technical challenges associated with the preparation of similar nanofluids, such as particles size and shape, solution ionic strength, NPs volume concentration, temperature etc. [24, 96, 97]. 
It remains a challenge and a subject of strong argument to identify clearly the heat transfer mechanisms in nanofluids: higher specific surface area of NPs, dispersion and electric charge, Brownian motion, interfacial layer, collision between particles and nanoclusters morphology.

A review of the latest developments came into a consensus that NPs aggregation is the most significant factor that dictates thermal transport enhancement [22, 25, 27]. The agreement was based on the fact that NPs motion while in solution results from all the interconnections involving van der Waals forces, columbic repulsion and steric action with the neighboring species. Therefore, balancing the various competing interactions leads to NPs dispersion and thus prevents aggregation and sedimentation [96, 98]. The ability to tune NP interactions, and thereby modulate the dispersion level, holds the key to rationally synthesize a stable nanofluid and extend the shelf-life of its properties and functionality. However, engineering nano-sized suspensions remain a challenging task both experimentally and theoretically. Recent empirical and quantum studies on interfacial phenomena shed light on the specific factors influencing the interNP and water-NP interactions, thereby clarifying the multiple, closely coupled processes controlling the interactions between NPs dispersed in water (i.e. dissolution, adsorption, protonation, hydroxylation, etc.). For instance, creating a high surface charge produces an electrical double-layer around the NPs, which results in strong repulsive Coulombic forces that promote particle dispersion [99].

The $\mathrm{pH}$ variation effects on dispersion stability and other properties of the alumina/water have been studied by several researchers [100-103]. G. Lefvre et al [100] calculated the real surface charge from titration experiments of hydrated -alumina suspensions for high and low $\mathrm{pH}$ values. According to their study, there is a large effect of dissolution on surface charges determination for $\mathrm{pH}<10$ and $\mathrm{pH}<4.5$ [100]. The correction for such an effect on alumina nanoparticles in acidic solution lead to an apparent saturation surface charge of $+2.3 \mathrm{at} / \mathrm{nm}^{2}$ (corresponding to $+0.37 \mathrm{C} / \mathrm{m}^{2}$ ) for a $\mathrm{pH}$ range less than 3.75. Lately, Mui et al. 25] investigated the aggregation and stability phenomenon of commercially available $\gamma-\mathrm{Al}_{2} \mathrm{O}_{3}$ NPs as a function of the solution $\mathrm{pH}$, ionic strength for better understanding of the colloidal behavior of $\gamma-\mathrm{Al}_{2} \mathrm{O}_{3}$ NPs in aqueous environments. According to their analysis, $\gamma-\mathrm{Al}_{2} \mathrm{O}_{3}$ NPs possess pH-dependent surface charges, with a point of zero charge (PZC) of 
$\mathrm{pH} 7.5$ to 8 for maximum aggregation. It has been also revealed that $\gamma-\mathrm{Al}_{2} \mathrm{O}_{3} \mathrm{NPs}$ attain a positively charged surface $(\zeta$ potential) in acidic solution $\mathrm{pH}(\mathrm{pH}<\mathrm{PZC})$ and a negatively charged surface with $\mathrm{pH}>\mathrm{PZC}[25]$.

According to the previously mentioned findings [96, 98, 103], it is essential to prepare nanofluids at low pH values, where the nanoparticles become positively charged, thus inducing repulsive forces between them and promoting a well-dispersed regime and maintaining the suspension stability [104, 105]. In fact, avoiding agglomoration and ensuring stability should be the main target to extend the shelf-life of nano-colloid properties and functionalities. More recently, Bouguerra et al. [27] examined the dispersion regime in alumina/water-based nanofluids by experimentally conducting simultaneous measurements of thermal conductivity and dynamic viscosity for different volume fractions of NPs immersed in different $\mathrm{pH}$ solution.

In response to the increased need for simulation and theoretical techniques induced by cost effectiveness and time-saving interest, a lot of theoretical work has been proposed to predict nanofluids thermal properties at different conditions [28, 106]. However, the initial predictions based on various assumptions rather than definite formulation accumulate ambiguity in the collection of results and generate wide inaccuracies. A consensus has yet to be reached when it comes to modelling the real microscopic behavior of heat transfer involved in nanofluid dispersion [23, 107].

Among other computational techniques, MD stands out as a highly promising alternative. From a conceptual standpoint, the approach provides inestimable atomic-level details to help us elucidate the intrinsic heat transport mechanisms in nanofluids [28]. Recently, Jabbari et al. [18] conducted a comprehensive review of the different MD methods applied to predict the thermophysical properties of nanofluids, mainly viscosity and thermal conductivity. They highlighted the gap between the different techniques and the missing components that need to be considered for future studies. Most of the MD findings on nanofluids found in the literature are skewed by a large number of simplifications and limitations [108 113]:

- They are restricted to modeling one NP thus disregarding the collective behavior of many NPs and their dipersive state 
- They fail to represent the real atomic structure and electrostatics at the solid-liquid interfaces which are highly altered due to hydroxylation, protonation/deprotonation phenomena from solution $\mathrm{pH}$ effects, etc.

- They use generalized mixing rules to calculate the potential energy parameters corresponding to the interactions between unlike species.

- They fall short to account for the actual inter-particle energies and electrostatics of nanoparticles scattered in acidic aqueous solutions.

The result of all these assumptions lead to incorrect predictions of the real interaction potential energies, the system dynamics and its macroscopic properties.

With the above background in mind, there is a need of a more reliable predictive MD model to uncover the critical links between nanoscale and macroscale phenomena, and facilitate a controlled suspension of the nanofluid.

$\gamma$-alumina nanomaterial was selected in this work due to the extensive number of related studies found in the literature and its broad use in many technological applications. Alumina is largely utilized for its relatively high thermal conductivity and largely employed in thermal nanofluids [4]. Moreover, the chemical resistance and porosity of alumina surfaces enable superior performance when it is used as a catalyst or catalyst support [27]. Additionally, aluminum oxide clusters have been extensively studied both numerically and experimentally to better understand the relationship between atomic structures, bonding nature and other interfacial surface properties with water [114-116]. Still, the interfacial properties are very challenging to obtain because they are highly dependent on the experimental conditions. For instance, due to possible ion migrations upon surface hydration, features like electronic and structural surface density make the actual chemical nature of the oxide surface intrinsically difficult to describe at the atomic level.

Thanks to recent quantum computations conducted on $\gamma$-alumina/water interfaces, a better atomic scale understanding of the structure and dynamic behavior of the surface atoms along with the interfacial water molecules was furnished. Recent studies thoroughly investigated the surface morphology and properties of hydrated $\gamma$-alumina resulting from various chemical phenomena that take place at the solidliquid interface such as dissolution, 
hydration, protonation, deprotonation and others [3, 5, 100, 117, 118].

A successful application of MD technique requires the use of a potential force field (FF) that effectively and accurately accounts for the interactions in complex colloidal systems [28]. That is, the force field must be transferable and suitable for effective modeling of the actual chemical nature (electronic and structural) of the metal oxide $\gamma-\mathrm{Al}_{2} \mathrm{O}_{3}$ surface and the liquid (water) in contact with the solid NPs. The Clay Force Field model, hereafter referred to as CLAYFF, was developed by Cygan et al. [3] and has shown to effectively model the behavior of clay/water systems in MD simulations [119]. It is based on an ionic, non-bonded, description of the metal-oxygen interactions associated with hydrated phases. The results obtained to date demonstrate that the CLAYFF shows good promise for MD simulations to accurately reproduce the interaction potential of hydrated aluminium-oxides, including correctly capturing the attractive van der Waals and the repulsive electrostatic interactions, as well as entropic effects which are particularly important at the nanoscale [100]. However, despite its success, few limitations have also become evident with the use of the original CLAYFF. In this study, the original CLAYFF model was augmented to account for positively charged alumina NPs to effectively describe the structure and behaviour of water, hydroxyl, protons and the surface atoms of alumina-waters in an acidic $\mathrm{pH}$ regime.

On the other hand, MD studies rarely couple the thermal conductivity enhancement and the viscosity variations of nanofluids. Yet, the enhancement can be very sensitive to the effective viscosity [26, 27]. To this end, it is crucial to assess the performance of heat transfer nanofluids by the combination of the properties rather than by each of the individual properties.

Due to shortage of microscopic understanding on NPs behavior from existing experimental and the lack of precision of numerical MD work to reproduce thermal transport, the present chapter responds to the necessity to adopt a precise MD approach capable of predicting the thermophysical properties (such as density, viscosity, heat capacity and thermal conductivity) of $\gamma-\mathrm{Al}_{2} \mathrm{O}_{3}$-water nanofluid suspended in acidic solutions. The main goal of this work is to conduct MD simulations with the focus on the manipulation of solid-liquid interfacial atomic structure and electrostatic state, in order to create positively charged alumina nanoparticles dispersed in water, thereby bringing a closer solution of delineating 
methods for modulating the stability and aggregation of nanofluids.

A hybrid potential field, comprised of refined force field models (extended CLAYFF and $\mathrm{SPC} / \mathrm{E}$ ), was first produced to allow a precise integration of the nanoscale phenomena into the dynamics of the suspended positively charged alumina NPs, thereby bridging the challenging gap between the nanoscopic solid-liquid interfacial phenomena and macroscopic thermodynamic properties.

Nest, the refined FF was synergistically combined to the MD model [3] to probe the thermophysical properties of the nanofluid evaluated at 4 different volumetric concentrations $(1 \%, 2 \%, 3 \%, 4 \%)$ and over a temperature range of 5 to $40{ }^{\circ} \mathrm{C}$ to be able to compare the data with the experimental findings of alumina/water for convective heat transfer applications existing in the literature 4]. We finally compared the performance efficiency of nanofluids to the corresponding basefluid by calculating the Mouromtseff number (Mo which serves as a factor of merit based on the four properties of the fluid: density $(\rho)$, dynamic viscosity $(\mu)$, thermal conductivity $(k)$ and heat capacity $\left(c_{p}\right)$ [26, 27]:

$$
\text { Mo }=\frac{\rho^{0.8} k^{0.67} c_{p}^{0.33}}{\mu^{0.47}}
$$

This chapter is organized as follows: a description of the the methodology details are outlined in section 6.2 . Then, section 6.3 provides an analysis of the validity of computational results pertaining to the thermodynamics and heat transfer enhancement of the nanofluid. Finally, the summary of our investigation and the pertinent conclusions are drawn in Section 6.4.

\subsection{Methodology}

The precision of MD simulations highly depends on the selection of a realistic force field model, system size and simulations timescales. Most MD studies conducted till date are skewed by many assumptions, which calls for enhanced MD models, in which the fewest number of simplifying assumptions are applied, on one hand, and the volume of computations is not increased significantly, on the other hand [28]. Put differently, in order to model thermophysical properties concordant with real experimental measurements, it is crucial to scout and implement the relevant findings in the literature to shape an accurate potential field capable of 1- emulating the complexity between the water and $\mathrm{Al}_{2} \mathrm{O}_{3} \mathrm{NPs}$ and 2- sustaining a dispersed regime corresponding to a $\mathrm{pH}<\mathrm{PZC}$ [100]. 


\subsubsection{Initial Configuration and Choice of Potential FF}

The chemical nature of the surface, its charge and the concentration and distribution of NPs are expected to play a key role in the heat transfer mechanisms. Therefore, it is vital to identify the initial system structure to reproduce realistic features of nanofluids observed under experimental conditions. Material Studio was initially used to confine the molecules in a cubic simulation box and optimize the systems initial structure [120]. The $\gamma-\mathrm{Al}_{2} \mathrm{O}_{3}$ particles were extracted by carving a sphere out of $\gamma-\mathrm{Al}_{2} \mathrm{O}_{3}$ supercell. The diameter of the $\mathrm{NP}$ was fixed at $4 \mathrm{~nm}$. Various coordination number of $\mathrm{Al}$ and $\mathrm{O}$ sites were found to be left at the surface. Undercoordinated Al-edge were healed with $\mathrm{H}_{2} \mathrm{O}$ groups (acidic level solution) whereas undercoordinated O-edge were healed with protons [100, 116, 117]. The well-dispersed state was modelled by initially placing the assembled NPs in the box at certain distances so that it has insufficient time to aggregate within the time domain [106]. The rest of the space in the cell was homogeneously occupied by water molecules. The water density was set to $1.0 \mathrm{~g} / \mathrm{mL}$ for all the starting configurations. The number of $\gamma-\mathrm{Al}_{2} \mathrm{O}_{3} \mathrm{NPs}$ were tuned to model the nanofluids with different volume concentrations. A snapshot of the simulation model is shown in Figure 6.1.

The simulation of dispersing nanoparticles is performed in a systematic way. The detailed steps are stated below:

1) Carving a spherical nanoparticle with a diameter of $4 \mathrm{~nm}$ from an optimized crystal unit cell of gamma-alumina (110) using Material studio software. The parent bulk gammaalumina structure for the slab model used in this work originates from the optimized model proposed by Ngouana-Wakou et al [5]. The optimized cell parameters consisted of a = $8.034 \mathrm{~A}, \mathrm{~b}=8.36 \mathrm{~A}$, and $\mathrm{c}=5.55 \mathrm{~A}$ containing 40 atoms $(16 \mathrm{Al}$ and $24 \mathrm{O})$.

2) Hydroxylation phenomena is assumed to occur on the surface chemistry of alumina NP when in contact with water molecules. Therefore, the obtained carved sphere is functionalized by further hydroxylating the dangling bonds found on the surface. More specifically, the dangling bonds were saturated with $\mathrm{H}$ and $\mathrm{OH}$ terminal groups such that their coordination number of the surface atoms will be the same as in the bulk crystal. 
(a)

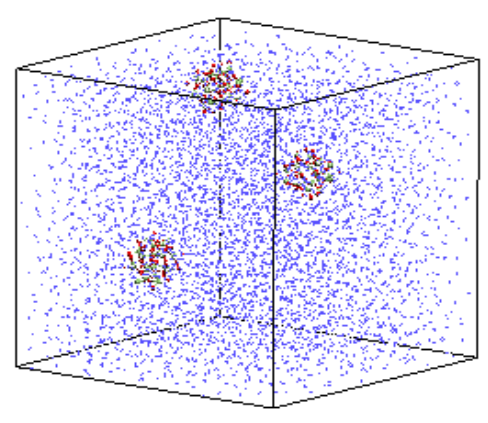

(c)

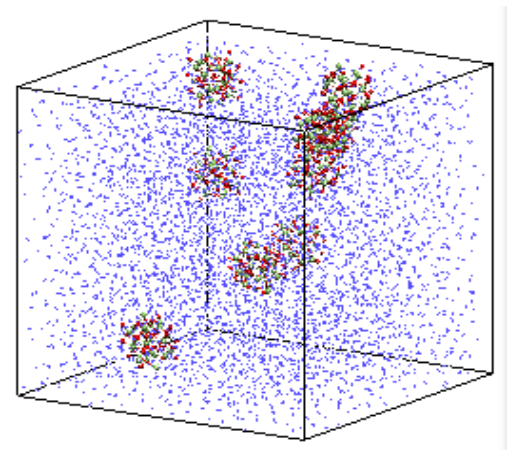

(b)

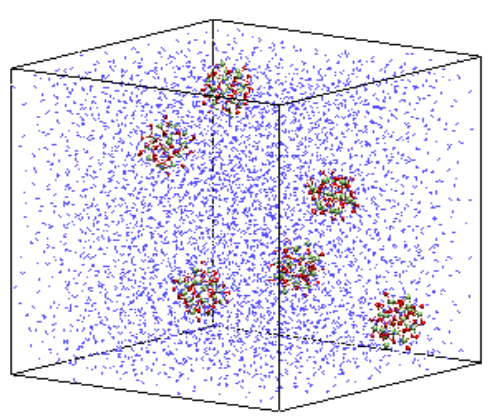

(d)

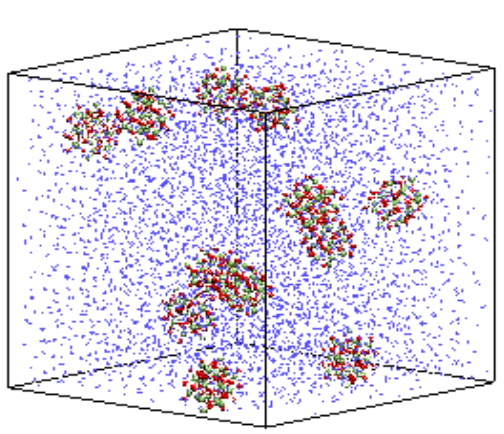

Figure 6.1: NPs dispersion morphology in water for (a) $\phi=1 \%$ (b) $\phi=2 \%$ (c) $\phi=3 \%$ (d) $\phi=4 \%$. Red and green represent oxygen and aluminum atoms of NPs and blue symbolizes water molecules (base fluid).

3) Protonation of surface hydroxyl by adding hydrogen bond to increase its surface charge and reach a value of $2.3 \mathrm{at} / \mathrm{nm} 2$.

\section{System and NP Sizes}

Modelling the conventional nominal size of one alumina NP (usually of a diameter $30 \sim$ $100 \mathrm{~nm}$ in most industrial applications) requires a large number of atoms in MD simulations. As a result, generating multiple NPs distributed in an aqueous solution requires an enormous number of water molecules. The separation in scales between the size of the water molecule and the NP renders the modelling of scattered nano-colloidal system very complex 
and demands extensive computational resources in MD.

Since the main goal of this study is to estimate the transport properties of nanofluid while visualizing the corresponding assembly at different low volumetric concentrations (1$4 \%$ ), it is essential to have enough number of NPs in the system and ameliorated prediction of the dynamic behaviour resulting from interparticle potentials specially at the solid-liquid interfaces. A compromise must be found between the targeted accuracy and the size of the models. Therefore, the NP diameter was fixed at $4 \mathrm{~nm}$ (after hydroxylation) for all the systems such that the minimum number of generated NPs is 3 at the lowest concentration level. Note that the spherical NP shape and size was assess based on having a surface area large enough to correspond to the highest possible hydroxylation coverage and eventually an apparent positively surface charge of approximately $+0.37 \mathrm{C} / \mathrm{m} 2$ of a fully protonated NP [100].

The choice to integrate a more precise potential field interactions over modelling the actual nanoparticles size was based on the assumption that the solid-liquid interfacial interactions have a larger influence on the thermal motion and diffusion of NPs in the base fluid and ultimately the system thermophysical properties.

\section{Hybrid Force Field Model}

The water molecules were simulated using the simple point charge/extended (SPC/E) model [121. The model is known to reproduce accurate structural and dynamic properties of bulk water. In order to reproduce precise particleparticle and waterparticle interactions, an extended CLAYFF model was implemented [3, 5, 100, 117, 118].

The results obtained to date demonstrate that CLAYFF shows good promise for MD simulations to accurately reproduce the interaction potential of hydrated aluminium-oxides, including correctly capturing the attractive van der Waals and the repulsive electrostatic interactions, as well as entropic effects which are particularly important at the nanoscale [3]. However, despite its success, few limitations have also become evident with the use of the original CLAYFF. In this study, the original CLAYFF model was augmented to account for positively charged alumina NPs to effectively describe the structure and behaviour of water, hydroxyl, protons and the surface atoms of alumina-waters in an acidic $\mathrm{pH}$ regime. 
Below are the main restrictions found in the original FF model along with the corresponding modifications adopted to solve the limitations:

- It assumes a net surface charge of zero (NPs at point zero charge (PZC)). Therefore, the charge distribution on the NP was altered from the classical CLAYFF force field by adding protons to the surface hydroxyl groups (adding $\mathrm{H}$ bonds to form $\mathrm{OH}_{2}$ groups) until a globally positive NP, with a surface charge density of $+0.37 \mathrm{C} / \mathrm{m}^{2}$ is reached.

- The difficulty to accurately represent the edges of finite size nanoparticle rather than infinitely layered periodic structures. Recently, Pouvreau et al. [2] proposed a systematic approach to solve this problem by developing specific metal-O-H (M-O-H) bending terms for CLAYFF, $E_{\text {bend }}=k\left(\theta-\theta_{0}\right)^{2}$ to better describe the structure and dynamics of singly protonated hydroxyl groups at mineral surfaces. Based on a series of DFT calculations, the optimal values of the Al-O-H for $\mathrm{Al}$ in octahedral coordination are determined to be $\theta_{0 A l O H}=110^{\circ}$ and $\mathrm{k}_{A l O H}=15 \mathrm{kcal} / \mathrm{mol} \mathrm{rad}^{2}$. The addition of the new bending term leads to a much more accurate representation of the orientation of O-H groups at the basal and edge surfaces [2].

Details on the parameters can be found in TableA.1 in the Appendix. The LJ parameters for unlike interactions were determined by Lorentz-Berthelot mixing rules. The cutoff distance for all interactions was 9 . The ParticleParticle Particle-Mesh K-space (PPPM) solver was applied for long range electrostatic interactions with a precision of 1/10,000 [34].

\subsubsection{EMD Simulations}

To validate the performance of the employed force field model, the density and specific heat capacity of the system were compared to the experimental results in [4]. EMD simulations were carried out at a fixed temperature of $300 \mathrm{~K}$ and pressure of $0.1 \mathrm{MPa}$. Periodic Boundary Conditions (PBC) were applied for all the nanofluid cases. The system configuration was initially brought to a local potential energy minimum by performing an energy minimization. Then, the NPT ensemble was employed for 4 different volume fractions ( $1 \%, 2 \%, 3 \%, 4 \%$ ),

with a Nose Hoover thermostat and barostat using time constants of 0.1 and 1 ps, respectively.

To ensure thermodynamic equilibrium, the convergence of total energy and its compo- 
nents (kinetic and potential; Coulomb, non-Coulomb, bonding, etc.) as well as temperature, density, and atomic radial distribution functions were carefully monitored during this equilibration period. Following the equilibration, the atomic trajectory was recorded during the next 40-100 ps of MD simulation at 0.004 ps intervals for the statistical analysis of the structural and dynamical properties of the simulated system.

All MD simulations in this work were performed using LAMMPS (Large-Scale Atomic Molecular Massively Parallel Simulator) software [30]. Long-range tail corrections to the energy and pressure were included [29]. The equations of motion were integrated using the velocity Verlet algorithm with a time step of 1 fs.

\subsection{Results and Discussions}

\subsubsection{Simulation Model Validation}

To ensure equilibrium state and convergence, the system energy and temperature fluctuations were carefully examined for the different NPs volume fraction. The total system energy and temperature exhibited a minor drift (less than $3.5 \%$ and $2 \%$ respectively) for all the cases, which verify that the MD simulation time is long enough to ensure accurate statistical calculation results.

\section{Density}

Figure 6.2 presents the variation of the nanofluid density as a function of the particle volumetric concentration and temperature. The present data of $\gamma-\mathrm{Al}_{2} \mathrm{O}_{3}$-water nanofluid can be seen to agree well with the experimental measurements obtained by Ho et al. [4]. It can be also verified that the density of alumina NPs is less sensitive to the temperature variation in comparison with the base fluid. 


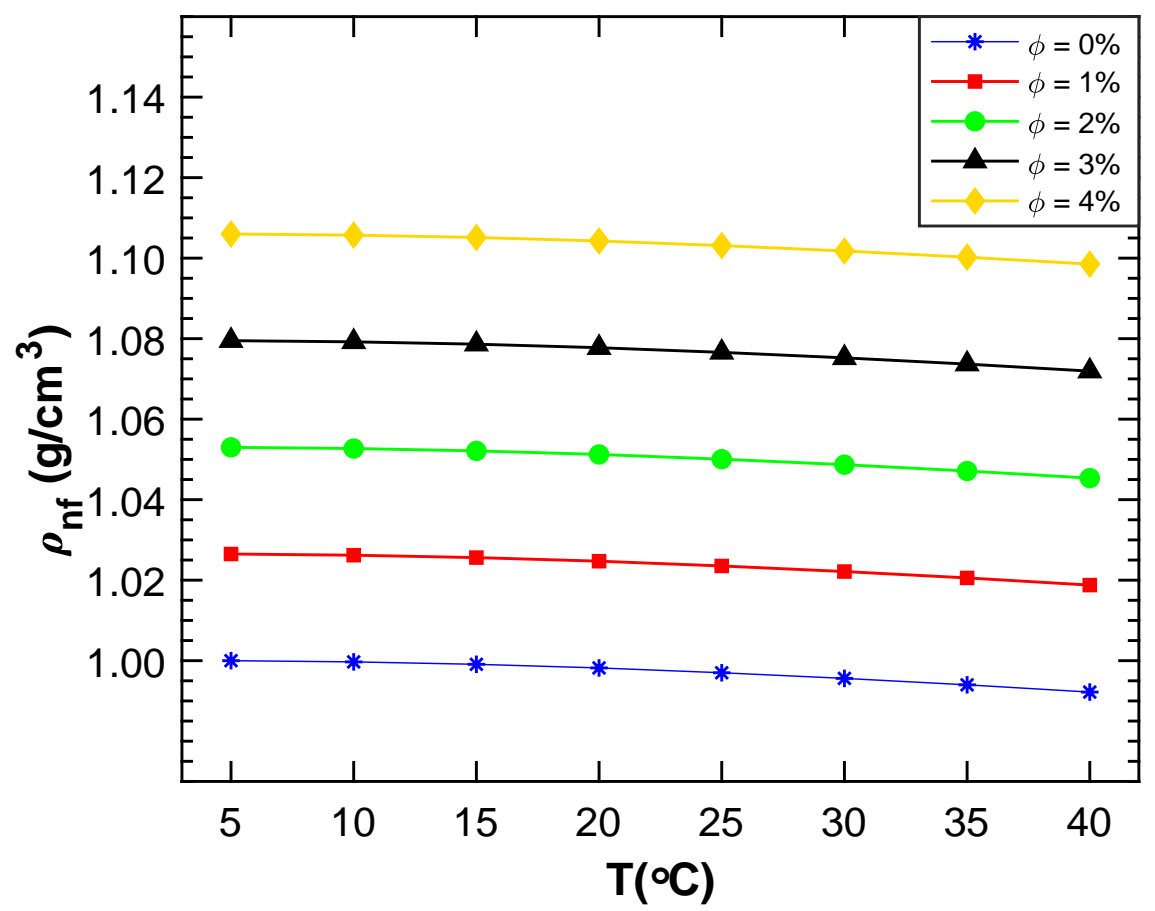

Figure 6.2: Variation of $\mathrm{Al}_{2} \mathrm{O}_{3}$-water nanofluid density with temperature for the different NPs volumetric concentration.

\section{Specific heat capacity}

Next, the specific heat capacity was also calculated using the following numerical formulation [122]:

$$
c_{p}=\frac{\left\langle E^{2}\right\rangle-\langle E\rangle}{K_{B} T^{2}}
$$

where, $\langle E\rangle$ is the average total energy, $T$ is the temperature and $K_{B}$ is Boltzmann's constant. During the simulation, fluctuations of the energy were recorded as a function of time $(t)$. Figure 6.3 indicates that the specific heat of the nanofluid decreases as the concentration of NPs increases, and does not change with the rise in temperature, which are prominently in line with experimental observations [4, 123]. The decrease of $c_{p}$ with increasing concentration can be attributed to the fact that alumina NPs have lower specific heat than water. Finally, the good agreement of the nanofluid density and $c_{p}$ with previous experiments [4] (5\% and 
$7 \%$ error respectively) verifies the accuracy of the employed MD computational scheme along with CLAYFF model, which succeeded in emulating the complex interactions between the base fluid and NPs.

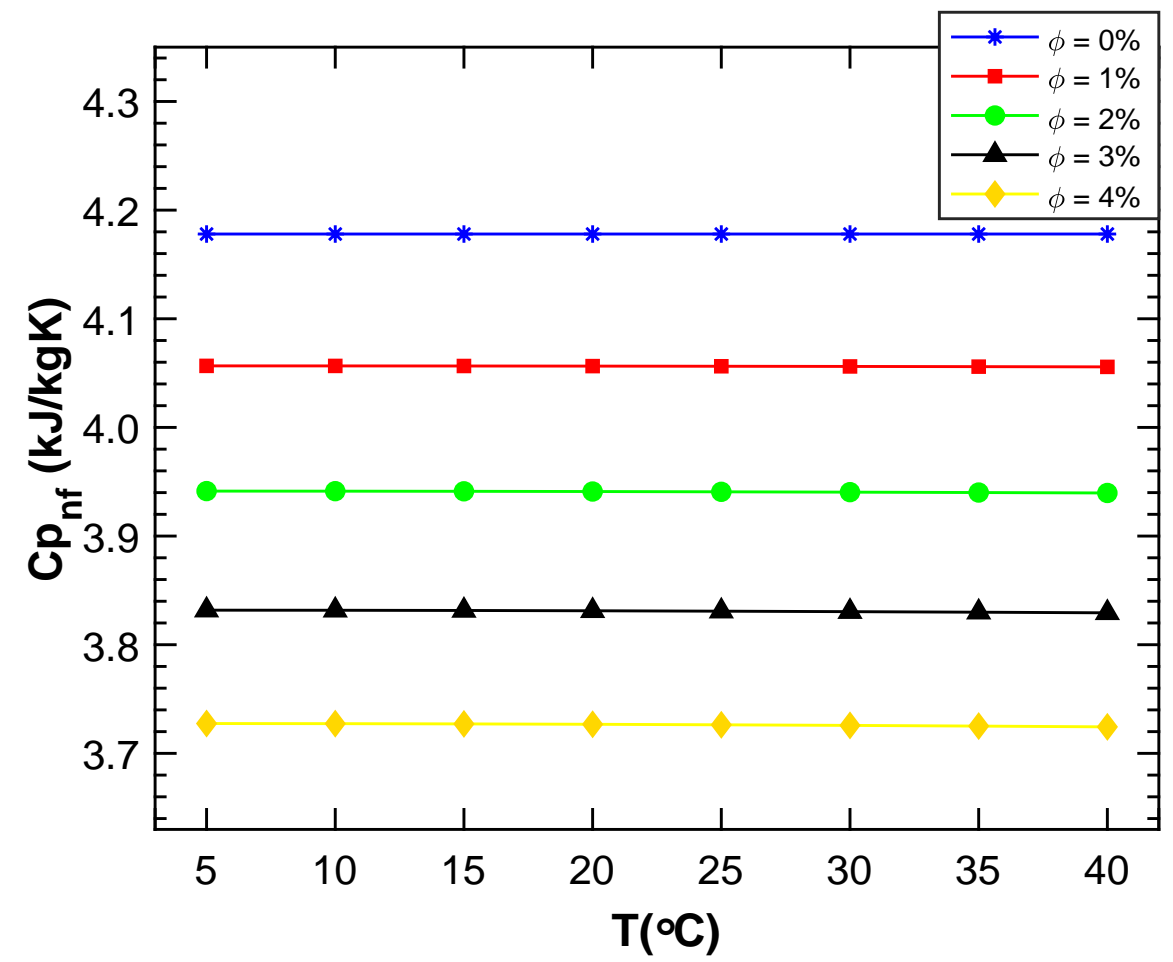

Figure 6.3: Variation of $\mathrm{Al}_{2} \mathrm{O}_{3}$-water nanofluid specific heat capacity with temperature for the different NPs volumetric concentration.

\subsubsection{Thermal conductivity and Viscosity calculation}

The Green-Kubo formulation was utilized to calculate the thermal conductivity $(k)$ and viscosity $(\mu)$ of the nanofluid. The values of $k$ and $\mu$ were obtained using the following equations respectively [28]:

$$
\begin{aligned}
& k=\frac{1}{3 K_{B} T^{2} V} \int_{0}^{\infty}\langle\vec{J}(t) \vec{J}(0)\rangle d t \\
& \mu=\frac{V}{K_{B} T} \int_{0}^{\infty}\left\langle P_{\alpha \beta}(t) P_{\alpha \beta}(0)\right\rangle d t
\end{aligned}
$$


where $K_{B}$ the Boltzmann constant, $\langle\vec{J}(t) \vec{J}(0)\rangle$ is the heat current autocorrelation function ( $\mathrm{HCACF}$ ) and $\left\langle P_{\alpha \beta}(t) P_{\alpha \beta}(0)\right\rangle$ is the stress autocorrelation function (SACF). According to the time evolution of HCACF and SACF (see Figure 6.4), a correlation length of 20 ps is sufficient enough to reach convergence and generate stable results of the nanofluid relative thermal conductivity and viscosity. Figure 6.4(a) and (b) represents the HCACF and SACF of the nanofluid evaluated at $\phi=2 \%$ and $\mathrm{T}=25{ }^{\circ} \mathrm{C}$ respectively. Generally, HCACF and $\mathrm{SACF}$ of liquid water decay to zero monotonically. However, the addition of NPs changed the decay into an oscillatory manner. The oscillation intensities of the autocorrelations became stronger with increasing particle concentration, resulting in an increase in thermal conductivity and viscosity in the nanofluid. These fluctuations can be attributed to the effect of solid-liquid interfaces in the system.
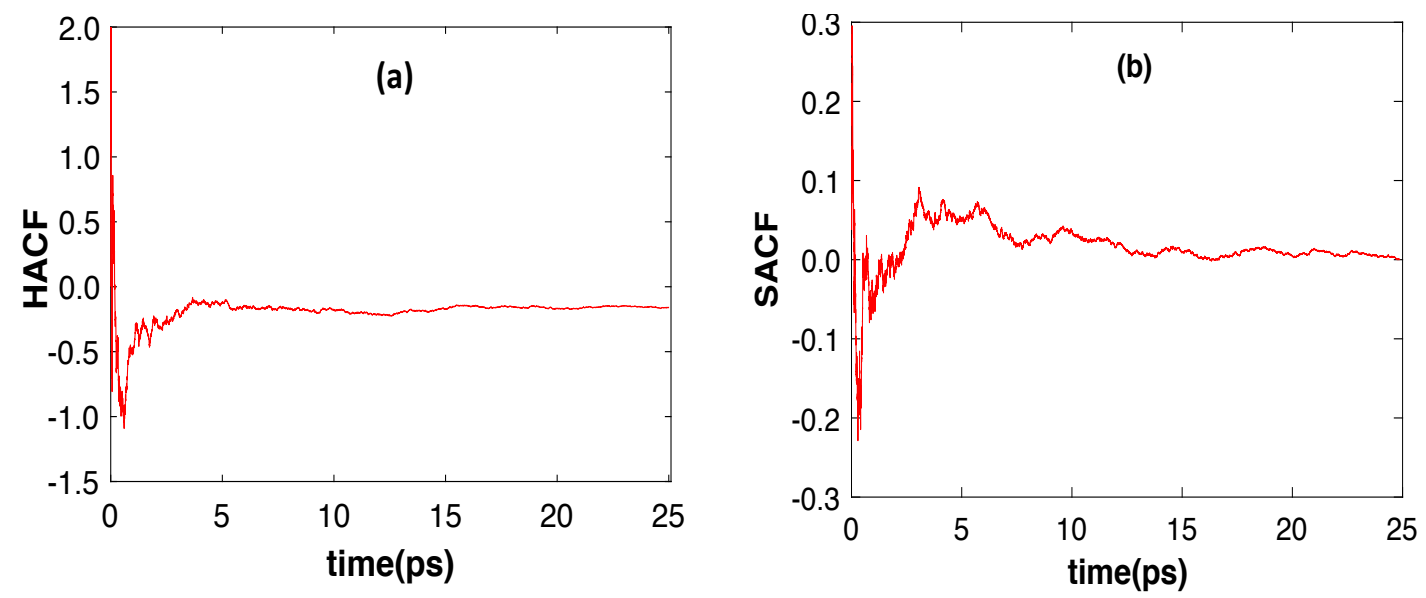

Figure 6.4: Time evolution of (a) HCACF and (b) SACF for the nanofluids at $\phi=2 \%$ and $\mathrm{T}=25^{\circ} \mathrm{C}$.

Figure 6.5 and 6.6 illustrate the dependence of alumina-water nanofluid viscosity $\left(\mu_{n f}\right)$ and thermal conductivity $\left(k_{n f}\right)$ on temperature (from 5 to $40{ }^{\circ} \mathrm{C}$ ) and volume concentration (from $1 \%$ to $4 \%$ ). According to Figure 6.5, the estimated viscosity of the nanofluid displays a trend of quiet steep rise with the particle fraction; in particular at $\mathrm{T}=15{ }^{\circ} \mathrm{C}$, a relative increase of up to $59 \%$ in the viscosity arises for the nanofluid containing $4 \%$ of NPs with respect to water (see Figure 6.7(a) for relative change in $\mu_{n f} / \mu_{b f}$ ). 


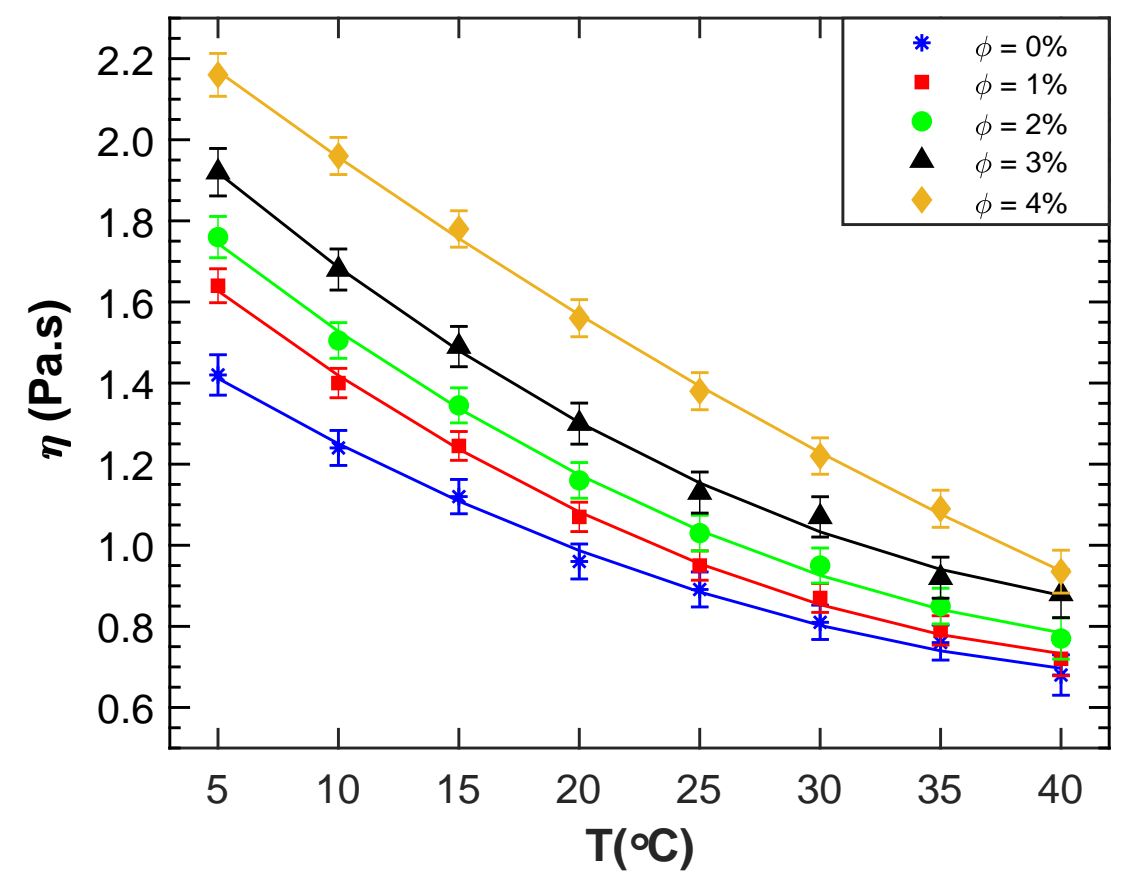

Figure 6.5: Variation of $\mathrm{Al}_{2} \mathrm{O}_{3}$-water nanofluid viscosity with temperature for the different NPs volumetric concentration.

This significant raise is most likely due to increased solid-liquid interfacial interactions when adding alumina NPs and the possibility of agglomeration in suspension, fostered by augmented intermolecular forces between the solid particles [26]. Moreover, from Figure 6.5. the decline in viscosity of the nanofluid with temperature becomes gradually pronounced as the particle fraction is increased. As can be expected, the relative increase in viscosity of nanofluid tends to be markedly reduced as the temperature increases. On the other hand, it is observed that the thermal conductivity increases with an increase in the particle volumetric concentration and also with an increase in the temperature. For example, at 25 ${ }^{\circ} \mathrm{C}$ the thermal conductivity is increased by about $21 \%$ with respect to the base fluid for a $4 \%$ volume fraction (see Figure 6.7 (b) for relative change in thermal conductivity $k_{n f} / k_{b f}$ ).

It is evident from the estimated trend of both viscosity and thermal conductivity that 


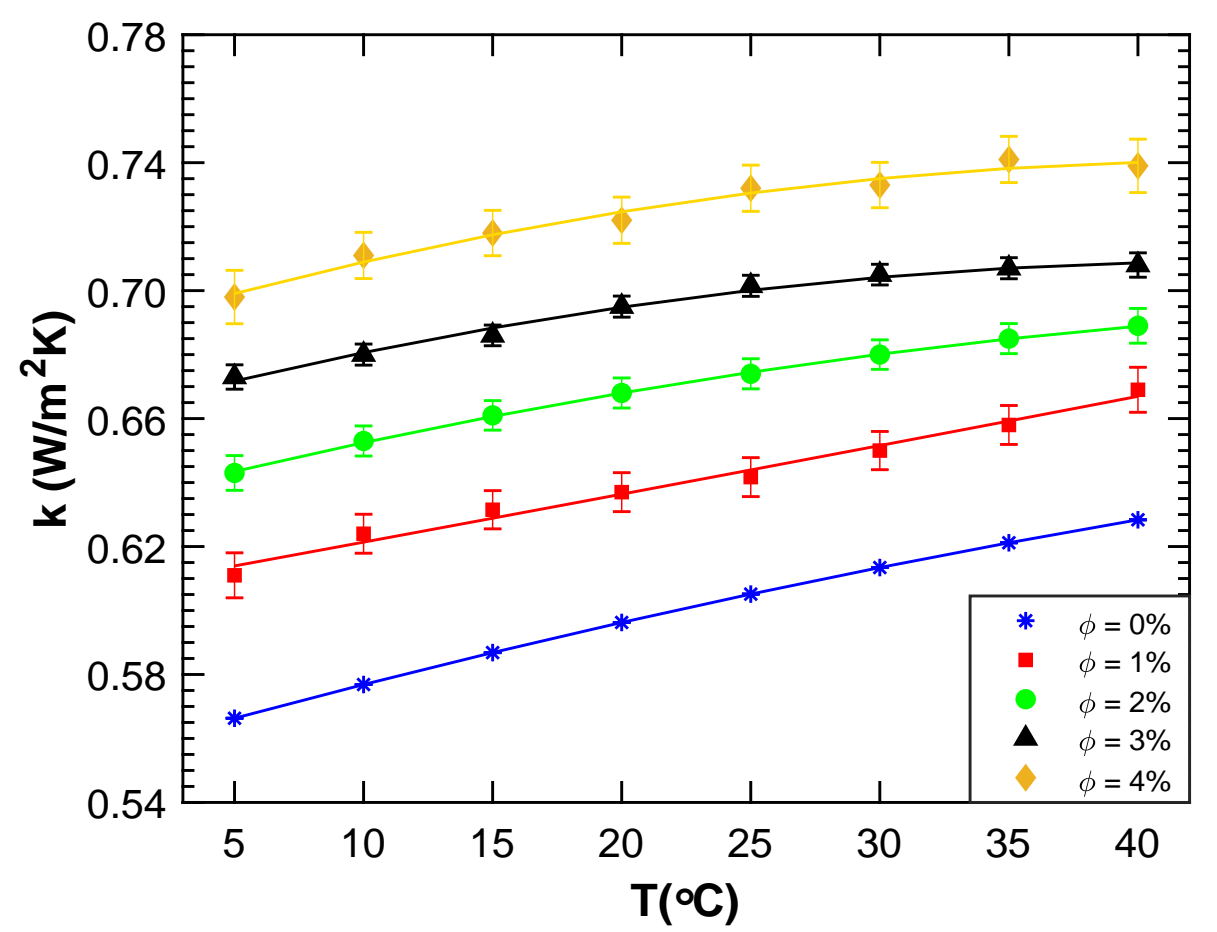

Figure 6.6: Variation of $\mathrm{Al}_{2} \mathrm{O}_{3}$-water nanofluid thermal conductivity with temperature for the different NPs volumetric concentration.

there exists a good agreement between the present MD results and the experimental data of Ho et al. 4]. Certainly, an increase of $\phi$ leads to the enhancement of thermal conductivity, accompanied by an increase of viscosity of the nanofluid as depicted in Figure 6.7. The dispersed NPs promote the improvement of thermal conductivity since they accentuate some benefits from a high specific surface, promoting the intermolecular interactions at the solidliquid interfaces. But also a higher concentration increases the probability of interparticles collisions which generate NPs aggregation and overall viscosity [27].

\subsubsection{Mouromtseff Factor (Mo)}

Given the opposite variations of thermal conductivity and viscosity, an explicit evaluation of the nanofluid heat transfer performance is crucial to present in order to recognize its behavior in promising thermal applications. Therefore, the Mouromtseff number Mo was calculated according to Eq. (6.1) to better identify the enhancement in nanofluids. Figure 

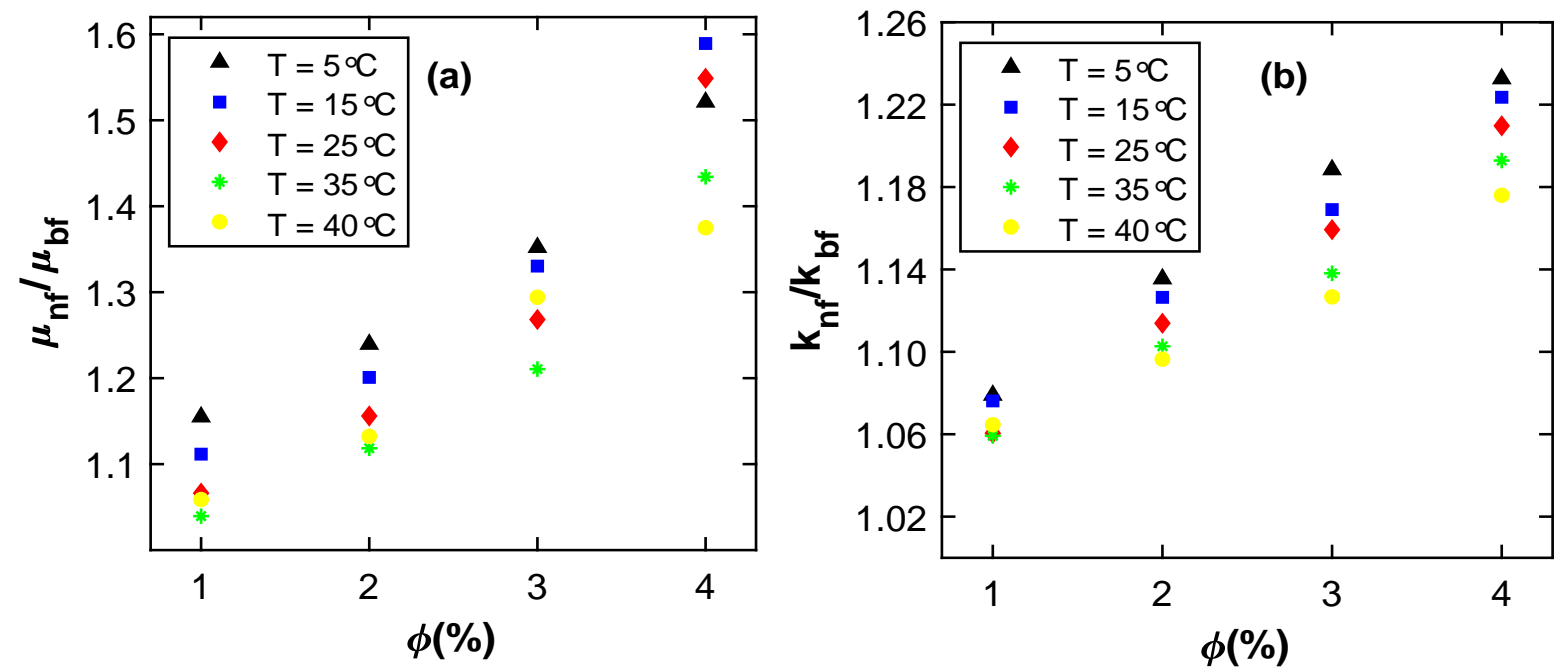

Figure 6.7: Relative change of nanofluid viscosity (left) and thermal conductivity (right) with respect to $\phi$ at different system temperatures.

6.8 shows the relative viscosity $\left(\mu^{*}\right)$, thermal conductivity $(k)$ and heat transfer rate $\left(\mathrm{Mo}^{*}\right)$ with respect to the base fluid versus temperature for each $\mathrm{Al}_{2} \mathrm{O}_{3}$ concentration level (see Eq. 6.5). Cases where Mo is less than 1 indicate that the nanofluid is superior to the base fluid. 

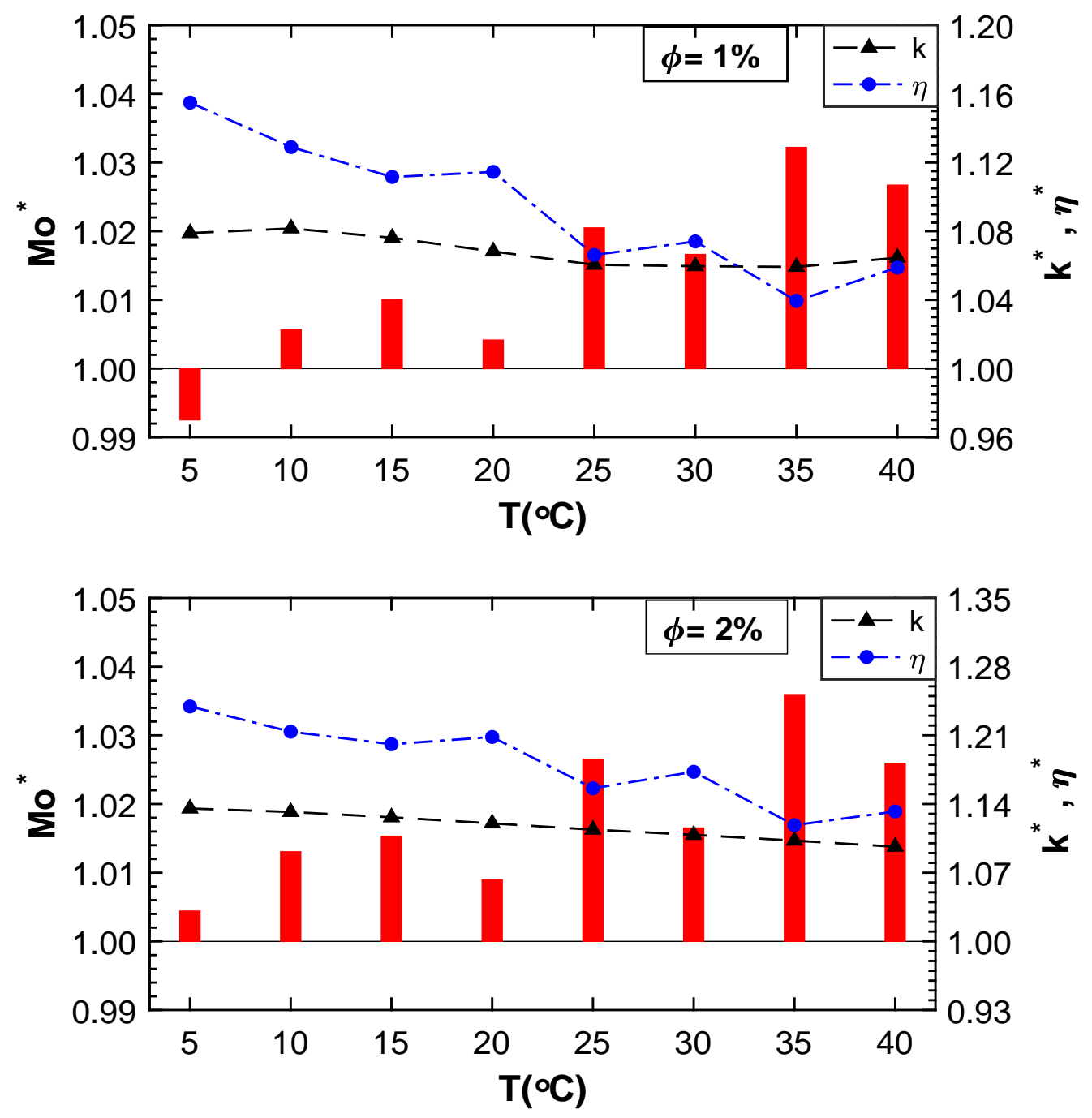

Figure 6.8: Heat Transfer efficiency ratio $\left(\mathrm{Mo}^{*}\right)$ and the corresponding thermal conductivity $\left(k^{*}\right)$ and viscosity $\left(\mu^{*}\right)$, ratios as a function of temperature for (a) $\phi=1 \%$ (b) $\phi=2 \%$ (c) $\phi=$ $3 \%$ (d) $\phi=4 \%$. 


$$
\mathrm{Mo}^{*}=\frac{\mathrm{Mo}_{n f}}{\mathrm{Mo}_{b f}} ; \quad k^{*}=\frac{k_{n f}}{k_{b f}} ; \quad \mu^{*}=\frac{\mu_{n f}}{\mu_{b f}} ;
$$

It turns out that the increase of $\phi$ does not lead to any significant improvement in $M o^{*}$. In fact, at $\phi=2 \%$ the nanofluid presents a better overall energy performance (see Figure 6.8(b)). As a matter of fact, when attaining a reasonably low viscosity, nanofluids offer better performance than with highest thermal conductivity and high viscosity. At $\phi=4 \%$, the heat transfer enhancement becomes almost $6 \%$ worse than the base fluid (observed at $20{ }^{\circ} \mathrm{C}$ ) as depicted in 6.8(d). It reveals a very high viscosity attained at this level which subsequently degrades the effect of higher thermal conductivity. The heat transfer efficacy of using nanofluid is certainly dependent on the net effects by the relative changes in the thermophysical properties of the nanofluid, comprising the thermal conductivity, viscosity, the specific heat capacity and density. Only the relative increase in thermal conductivity contributes to the heat transfer enhancement, while the relative changes in the remaining properties (prominently the viscosity) affect unfavorably. In this case, replacing water by the nanofluid is inferred to be infeasible. Of course, high thermal conductivity is needed for maximum heat absorption in thermal transport applications. Nevertheless, low viscosity is also essential to ensure less power consumption and a better overall efficiency of the system.

There is no direct correlation between the heat transfer enhancement and its temperature and concentration levels (Figure 6.9). For instance, at $\phi=2 \%, \mathrm{Mo}^{*}$ reaches its maximum value at $\mathrm{T}=35{ }^{\circ} \mathrm{C}$, indicating that the nanofluid is more promising for higher temperature heat transfer applications [19, 26, 124]. These observations can be correspondingly related to the state of dispersion attained in each NPs concentration and temperature level.

This chapter results demonstrate the crucial role played by the repulsive electrostatic forces yielding well-dispersed NP suspensions, specially at low $\phi$. The NP surface charge density have shown great potential to stabilize NP suspensions, as demonstrated in some reported experimental papers, thereby qualitatively validating our modelling approach. Firstly, suspensions having higher $\phi$ exhibited a faster aggregation kinetics by showing a steeper increase of viscosity. The post analysis of Mo demonstrated that at lower $\phi$, the system show a higher propensity for stability and enhancement specially at high temperatures. On the 
contrary, for volume fractions higher than $2 \%$, the system thermal performance deteriorates which is speculated by the fact that the system exhibit a critical condition of aggregation and clogging, thereby establishing a threshold for the nanofluid functionality. The results have shown that the MD model developed here demonstrates an improved numerical effort for predicting the thermophysical properties of alumina/water while maintaining a precise representation of the atomic topology and interfacial potential interactions.

When characterizing the nanofluid, the thermal conductivity cannot be considered alone as a criterion for nanofluid selection. Viscosity of nanofluids is also essential, and it is possible that nanofluids with lower thermal conductivity enhancement may be preferred because they offer lower viscosity due to lower NPs concentration and a better dispersal state in the base fluid [26]. Thus, the optimal performance of nanofluid is when the thermal conductivity is high enough to induce a greater heat absorption with respect to the base fluid but low enough to maintain a minimal viscosity.

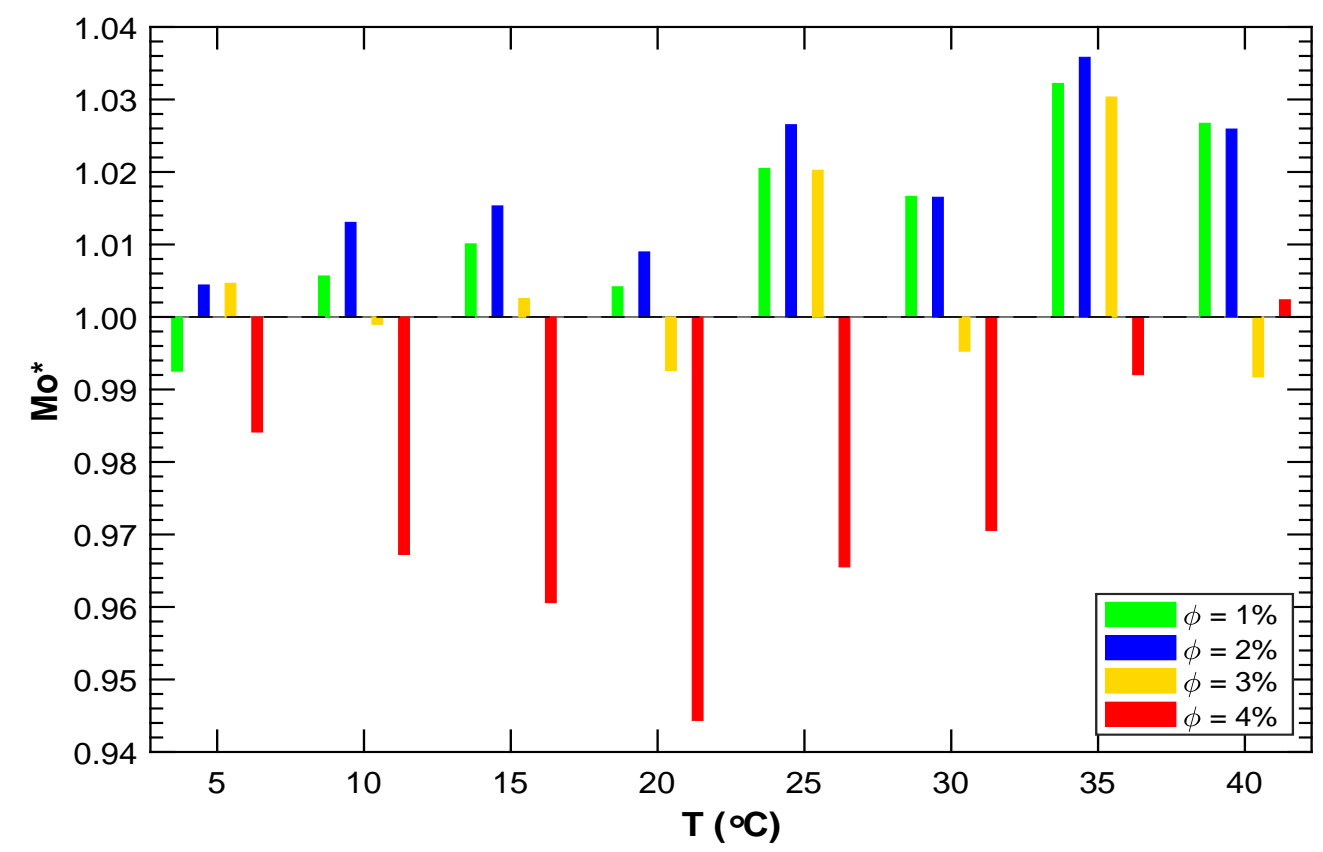

Figure 6.9: Global illustration of the heat transfer enhancement ratio computed at different $\phi(\%)$ and $\mathrm{T}\left({ }^{\circ} \mathrm{C}\right)$ conditions. 


\subsection{Conclusion}

The suspension of nanoparticles (NPs) in aqueous solutions, known as nanofluid, hold promise in many energy applications. Preventing the agglomoration/clustering of NPs in the base fluid while achieving a better enhancement of the overall thermophysical properties, has been a primary focus to ensure the stability and an extended shelf-life of nanofluids in many thermal applications.

The ability to tune NP interactions, and thereby modulate the dispersion level, holds the key to rationally synthesize a stable nanofluid and extend the shelf-life of its properties and functionality. However, engineering nano-sized suspensions remain a challenging task both experimentally and theoretically. Recent empirical and quantum studies on interfacial phenomena shed light on the specific factors influencing the interNP and water-NP interactions, thereby clarifying the multiple, closely coupled processes controlling the interactions between NPs dispersed in water (i.e. dissolution, adsorption, protonation, hydroxylation, etc.). In low $\mathrm{pH}$ range, the hydrated gamma-alumina surface undergoes a complete protonation and becomes positively charged, thus inducing repulsive coulombic forces between NPs and promoting the nanofluid stability by avoiding agglomoration, clogging and sedimentation [25, 100].

The existing MD work have unveiled numerous impediments in terms of reproducing the thermal transport phenomena in nanofluidic systems due to many simplifying assumptions in the potential field that neglect the effects predominant at the solid-liquid interface. Additionally, they miss to couple the thermal conductivity and dynamic viscosity and give an overall assessment of the thermal enhancement aimed for specific energy applications.

With the need of a more reliable predictive MD model to uncover the critical links between nanoscale and macroscale phenomena, and facilitate a controlled suspension of the nanofluid, we presented an improved MD approach to describe the dynamics and thermal transport behavior of alumina-water nanofluid found in acidic regimes (pH range $3 \sim 3.75<<$ PZE) and predict their overall performance. More specifically, the enhanced MD approach is synergistically combined to fortified force field models (extended CLAYFF and SPC/E) to accurately predict the thermophysical properties of alumina-water evaluated at different 
volumetric concentrations and temperatures while ensuring a stable nanofluid functionalities.

The MD results of the nanofluids properties were in agreement with the experimental trends observed in the work of Ho et al. [4]. An increase of $\phi$ lead to the enhancement of the thermal conductivity along with an increase of viscosity of the nanofluid. Therefore, a strong dependence of both, viscosity and thermal conductivity of alumina/water upon temperature and volumetric concentration was perceived. Consequently, the heat transfer enhancement was tested by evaluating the Mouromtseff number Mo [27]. It was concluded that the usefulness of nanofluids for heat transfer applications depends not only on the thermal conductivity, but also on other thermophysical properties, such as viscosity, density and specific heat capacity.

The results emphasize the intrinsic role played by the repulsive electrostatic forces yielding well-dispersed nanofluid, specially at low $\phi$. The NP surface charge density have shown great potential to stabilize NP suspensions. The post-assessment of the overall thermal performance predicts that at a lower $\phi$, the system shows a higher propensity for stability and enhancement specially at high temperatures. On the contrary, for $\phi$ higher than $2 \%$, the thermal enhancement deteriorates, speculated by the fact that the system exhibit a critical condition of aggregation and clogging, thereby establishing a threshold for the nanofluid functionality. In conclusion, the MD model developed here demonstrates an improved numerical effort for predicting the thermophysical properties of alumina/water while maintaining a precise representation of the atomic topology and interfacial potential interactions.

Further MD numerical investigations would be valuable to assess other effects like the addition of stabilizing agents, nanoparticle size as well as non-equilibrium thermal transport due to external fluxes could be also considered and included in future works to investigate with the proposed MD model a wider sampling of alumina-water nanofluid. 


\section{Chapter 7}

\section{Conclusions and Future Research Directions}

In this final chapter, a summary of the work completed and the research challenges faced throughout this project are presented. Avenues for possible future directions based on our contributions are also proposed.

As mentioned earlier, the central drive of this dissertation is to elucidate the underlying physics of thermodynamics in selected fluids of industrial interest by performing MD simulations based on efficient numerical schemes and reliable potential field models. This thesis demonstrated the value of particular MD cases capable of reproducing the transport behavior in complex mixtures, mainly hydrocarbon reservoirs and alumina-water nanofluid, at different conditions.

\subsection{Contributions}

\subsubsection{Thermodiffusion in Binary n-alkanes}

The first part of this thesis consists of introducing an enhanced non-equilibrium MD approach, comprised of the eHEX algorithm coupled with the TraPPE-UA force field. To ensure that the behavior observed in our NEMD simulations is representative of the real dynamical system, the selection of an accurate force field is essential. We first demonstrated 
that TraPPE-UA force field accurately reproduces experimental density and self-diffusion coefficients of linear molecules in multi-component systems. These properties are significant, since they govern the dynamics of the system species and directly influence their thermodiffusive behavior.

The validity of the newly employed method in quantifying the thermodiffusive motion was first tested for a binary mixture. The calculated Soret coefficients of the n-pentane/n-decane mixture at three different compositions showed a good agreement with the benchmarked experimental data and a better accuracy compared to other MD findings. The numerical outcomes verifying the competence of our improved approach are summarized below:

1. The computational precision of the eHEX along with the UA force field manages to accurately describe the molecular interactions in n-alkanes for the various compositions. Moreover, our MD approach achieved local equilibrium with great convergence while significantly conserving the system energy with minor instabilities;

2. The continuity of the three gradients (temperature, composition and mixture density) emphasizes that the system behavior, despite the extreme gradients, is still in the domain of linear response and converges with minimal computational errors;

3. The evaluation of our numerical results, when compared to other MD methods, clearly underlines the importance of using meticulous integration schemes and potential parameters capable of reproducing precise liquid properties with minimal truncation errors and computational cost.

\subsubsection{Thermodiffusion in Ternary n-alkanes}

Secondly, this work interests were expanded in ternary hydrocarbon mixtures to gain a better insight of the thermodiffusive behavior of composites in a more complex mixture, under a high pressure and a high temperature. Using the same eHEX approach, we evaluated the sign and magnitude of thermodiffusion separation ratio of methane/n-butane/n-dodecane ternary mixtures at five different compositions. The pertinent conclusions of our investigation are the following:

1. The method succeeded in revealing the coupled effect of relative density and mole fraction of the mixture species on thermodiffusion process; 
2. The separation ratio of the ternary mixture showed a good agreement with experimental data and better accuracy in predicting the sign change of the intermediate component (n-butane) as its concentration increases, when compared to other MD models.

\subsubsection{Thermal Transport of Nanofluids}

For another application, an enhanced MD algorithm was also applied to study thermal transport of nanofluids. Preventing the agglomeration/clustering of NPs in the base fluid while achieving a better enhancement of the overall thermophysical properties, has been a primary focus to ensure the stability and an extended shelf-life of nanofluids in many thermal applications. A manipulation of the solid-liquid interface chemistry in combination with the adjustment of NPs volumetric concentration is a promising strategy to modulate the dispersion level and prevent clogging, sedimentation and ultimately the deterioration of the nano-colloidal characteristics.

Experimental and numerical efforts have been failing to do so due to the lack of a deeper understanding of the influence of NP surface chemistry on the stability of nanofluids. For these reasons, there is a need of a more reliable predictive MD model to uncover the critical links between nanoscale and macroscale phenomena, and facilitate a controlled suspension of the nanofluid. Therefore, we produced an improved MD approach fortified with a refined hybrid force field model (extended ClayFF and SPC/E), revamped to deepen the understanding of interparticle potentials (i.e. cross-interactions between $\mathrm{Np}-\mathrm{Np}$, Np-water and water-water) and reveal the characteristics of interfacial atoms to ultimately establish a reliable prediction of the topology of fully protonated NPs dispersed in the base fluid, as well as the nanofluid macroscopic properties. The highlights of nanofluid MD findings are the below:

1. The MD predictions of the nanofluids thermophysical properties were in agreement with the experimental data. An increase of the NPs volumetric concentration $(\phi)$ lead to the enhancement of thermal conductivity along with an increase of viscosity.

2. The results demonstrate the crucial role played by the repulsive electrostatic forces yielding well-dispersed NP suspensions, specially at low $\phi$. The NP surface charge density have shown great potential to stabilize NP suspensions, as demonstrated in some reported experimental papers, thereby qualitatively validating our modelling approach. 
Firstly, suspensions having higher $\phi$ exhibited a faster aggregation kinetics by showing a steeper increase of viscosity.

3. The post analysis of Mouromtseff number demonstrated that at lower $\phi$ (less than $2 \%$ ), the system show a higher propensity for stability and enhancement specially at high temperatures. On the contrary, for volume fractions higher than $2 \%$, the system thermal performance deteriorates which is speculated by the fact that the system exhibit a critical condition of aggregation and clogging, thereby deteriorating the nanofluid thermal functionality.

4. The results have shown that the MD model developed here demonstrates an improved numerical effort for predicting the thermophysical properties of alumina/water while maintaining a precise representation of the atomic structure/charge along with interfacial potential interactions.

In summary, MD simulations blended with robust potential field models, allow the deliberate adjustment of various system parameters, to build an understanding of the relation between atomic behavior and macroscopic properties under favorable simulation conditions. It is expected that, as the power of readily obtainable computational resources increases and accurate force fields are devised, MD will start to play a substantial tool in many experimental labs for a broad range of thermal engineering and nanotechnology investigations.

\subsection{Challenges Faced}

In completing this work, we faced many obstacles and computational limitations. In this section, we identify several facets of the work that need to be further explored:

1. The most stringent limitation of MD simulations is the availability of a well-established, transferable potential field. As we use generalized combining rules, the accuracy of predictions declines with the structural complexity and asymmetry (type, phase, partial charge) of the different species composing the mixture. However, functional forms that are simpler and computationally more efficient than some existing models are still achievable;

2. Another drawback with MD is that it is computer-intensive, with typical simulations lasting hours or days rather than seconds or minutes. Systems displaying long relaxation 
times or presenting heterogeneities on a scale larger than 5- 10 nanometers are very difficult to simulate at the molecular level;

3. To avoid anomalous physical results in non-ideal systems, accounting for the average partial enthalpy in the microscopic heat flux calculation of EMD is crucial, since associated energy can be exchanged with diffusing particles [31].

As the review in Chapter 6 indicates, the methods developed in this thesis have not yet been pushed to their limits:

1. More numerical studies should be done to provide a better insight into the flow physics by considering NP behaviors and interactions in laminar, transition and turbulent regimes;

2. MD can only afford simulations for a very small domain. Solving Newtons equation of motion for a large system is quite time-consuming. Therefore, employing hybrid techniques that combine continuum fluid dynamics and MD simulation would be ideal;

3. Near thermally active surfaces, one can expect spatial variations of local thermal properties (viscosity, density, specific heat and thermal conductivity) due to induced accumulation or depletion of the NPs, which suggests other dynamics, such as thermodiffusion, might play a role on the heat transfer enhancement.

Once such improvements are unlocked, confidence will augment in conducting efficient performance investigations of nanofluids.

\subsection{Recommendations for Future Work}

A careful application of MD simulations, in concert with complementary experimental methods, represents an area of great opportunity in thermal science, and beyond. This opportunity will only grow as simulations become faster, cheaper, more widely accessible and more accurate.

The most obvious extension of nanofluid work area is to continue exploring its pivotal concepts for the synthesis of a new class of nano-engineered fluids with unique properties and applications. For instance, engineering a hybrid nanofluid by mixing various types of nanoparticles and phase change materials (PCM), can bring new opportunities for heat 
transfer enhancement through a manipulation of the system thermal properties and adjustment of the constituents concentration fractions;

Despite significant advances, no comprehensive model for nanofluid characteristics can be reached. To bridge the research gaps, the below guidelines are proposed as possible avenues to improve the reliability of simulations and extend the scope of thermal issues that can be tackled with nanotechnology:

1. It is essential to have a control over NP agglomeration and regulate their surface layers, which greatly affect nanofluids properties. Such control can be achieved by gaining a careful knowledge of the structuration and dynamics of atoms at the solid-liquid interface;

2. The development of a congruent factor of merit that targets practical thermal fluid applications should be a focus of future research, to address the coupled effects of the thermophysical properties.

3. A further avenue would be investigating $\mathrm{NP}$ suspensions at $\mathrm{pH}$ level higher than PZC (in basic solutions where NPs surface charge is negative) and ultimately evaluate the performance of nanofluids to build guidelines for their rational design and the modulation of their stability in different $\mathrm{pH}$ regimes. 
Appendix A

Molecular Dynamics Evaluation of Alumina Nanofluid Thermal Transport Performance 
A.1. Hybrid Potential Energy Field Parameters (extended CLAYFF and SPC/E)

Table A.1: Force field parameters implemented in this work [2, 3]

\begin{tabular}{|c|c|c|c|}
\hline \multicolumn{4}{|c|}{ Nonbonding Parameters } \\
\hline & $\mathrm{R}_{0}(\mathrm{~A})$ & $\mathrm{D}_{0}(\mathrm{kcal} / \mathrm{mol})$ & $\mathrm{q}(\mathrm{e})$ \\
\hline $\mathrm{Al}$ oct (bul/surface) & 4.7943 & $1.3298 \times 10-6$ & 1.575 \\
\hline $\mathrm{Al}$ tet (bul/surface) & 3.7064 & $1.8405 \times 10-6$ & 1.575 \\
\hline Oh (bulk/hydroxyl)a & 3.5532 & 0.1554 & -1.05 \\
\hline H (hydroxyl)a & 0 & 0 & 0.425 \\
\hline Ow (water)b & 3.5532 & 0.1554 & -0.82 \\
\hline $\mathrm{Hw}$ (water)b & 0 & 0 & 0.425 \\
\hline \multicolumn{4}{|c|}{ Bonding Parameters } \\
\hline & $\mathrm{r}_{0}(\mathrm{~A})$ & \multicolumn{2}{|c|}{$\mathrm{k}_{\mathrm{b}}\left(\mathrm{kcal} / \mathrm{mol} / \mathrm{A}^{2}\right)$} \\
\hline$|\mathrm{Ow}-\mathrm{Hw}|$ & 1 & \multicolumn{2}{|c|}{554.1349} \\
\hline |Oh-Hh| & 1 & \multicolumn{2}{|c|}{554.1349} \\
\hline & $\theta_{0}(\operatorname{deg})$ & \multicolumn{2}{|c|}{$\mathrm{k}_{\theta}\left(\mathrm{kcal} / \mathrm{mol} / \mathrm{rad}^{2}\right)$} \\
\hline Al-Oh-Hh & 110 & \multicolumn{2}{|l|}{15} \\
\hline $\mathrm{Hw}-\mathrm{Ow}-\mathrm{Hw}$ & 109.47 & \multicolumn{2}{|c|}{45.7696} \\
\hline
\end{tabular}


A.2. Comparison of MD predictions with Exp. and theoretical data of the nanofluid viscosity and thermal conductivity ratio at $\mathrm{T}=25^{\circ} \mathrm{C}$.
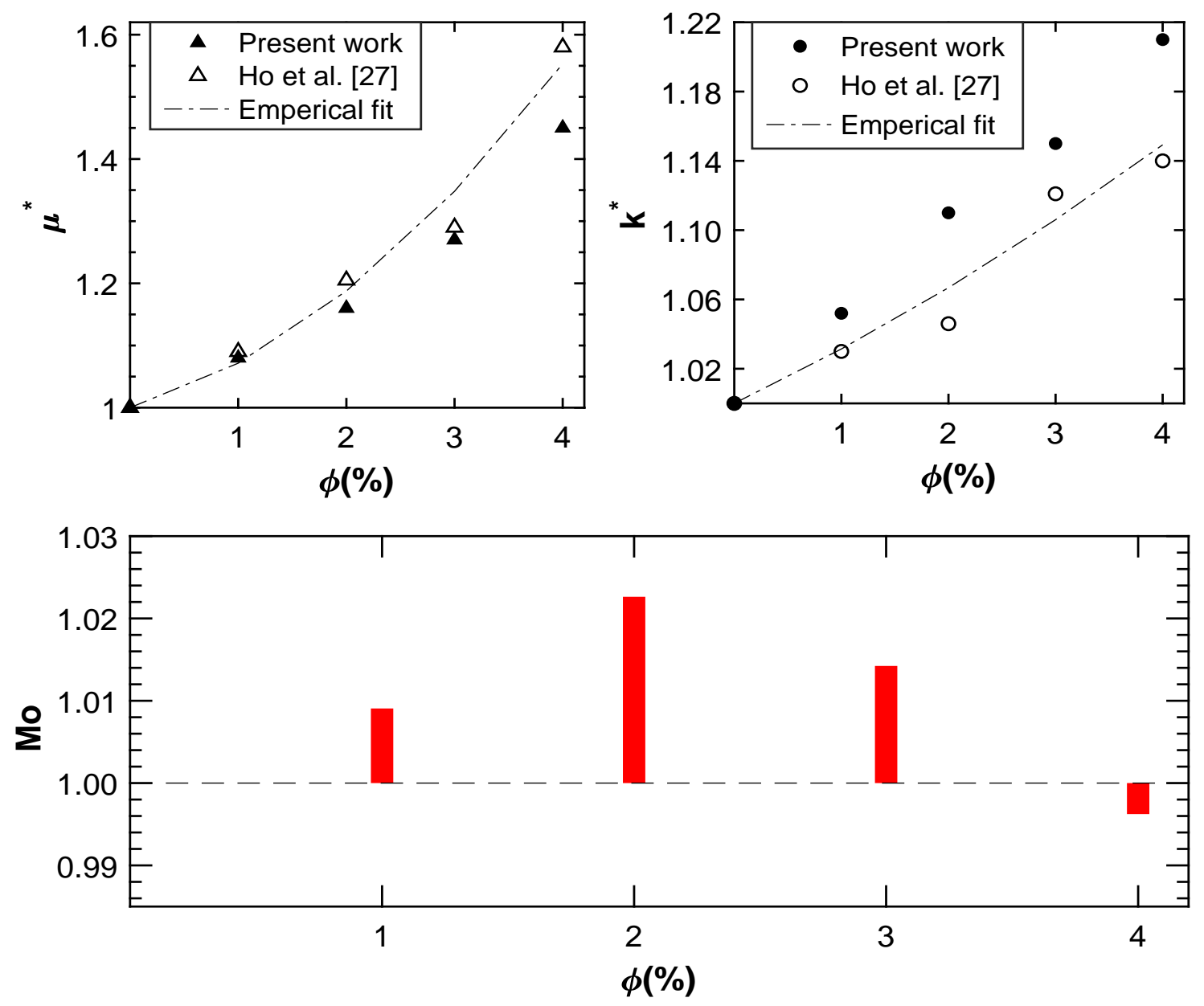

Figure A.1: Variation of the nanofluid viscosity ratio (left) and thermal conductivity ratio (right) as a function of $\phi$ for $\mathrm{T}=25^{\circ} \mathrm{C}$. 


\section{Bibliography}

[1] M. Z. Saghir, "Estimation of thermodiffusion in ternary alkane mixtures using molecular dynamics simulations and an irreversible thermodynamic theory," High Temp. High Pressures, vol. 38, p. 315, 2010.

[2] M. Pouvreau, J. A. Greathouse, R. T. Cygan, and A. G. Kalinichev, "Structure of Hydrated Gibbsite and Brucite Edge Surfaces: DFT Results and Further Development of the ClayFF Classical Force Field with Metal-O-H Angle Bending Terms," Journal of Physical Chemistry C, vol. 121, pp. 14757 - 14771, 2017.

[3] R. T. Cygan, J.-J. Liang, and A. G. Kalinichev, "Molecular Models of Hydroxide, Oxyhydroxide, and Clay Phases and the Development of a General Force Field," The Journal of Physical Chemistry B, 2004.

[4] C. J. Ho, W. K. Liu, Y. S. Chang, and C. C. Lin, "Natural convection heat transfer of alumina-water nanofluid in vertical square enclosures: An experimental study," International Journal of Thermal Sciences, 2010.

[5] B. F. Ngouana-Wakou, P. Cornette, M. Corral Valero, D. Costa, and P. Raybaud, "An Atomistic Description of the $\gamma$-Alumina/Water Interface Revealed by Ab Initio Molecular Dynamics," Journal of Physical Chemistry C, vol. 121, no. 19, pp. 10351$10363,2017$.

[6] A. Ghadimi, R. Saidur, and H. S. Metselaar, "A review of nanofluid stability properties and characterization in stationary conditions," International Journal of Heat and Mass Transfer, 2011.

[7] S. Srinivasan and M. Z. Saghir, Thermodiffusion in Multicomponent Mixtures. 2013. 
[8] S. Antoun, M. Z. Saghir, and S. Srinivasan, "An improved molecular dynamics algorithm to study thermodiffusion in binary hydrocarbon mixtures," The Journal of Chemical Physics, vol. 148, p. 104507, mar 2018.

[9] G. Galliero, "A new model for thermal diffusion: Kinetic approach," J. Am. Chem. Soc., vol. 130, p. 10963, 2008.

[10] S. Plimpton, "Fast parallel algorithms for short-range molecular dynamics," Journal of Computational Physics, 1995.

[11] D. Frenkel and B. Smit, Understanding Molecular Simulation: From Algorithms to Applications. 2002.

[12] F. Montel, "Phase equilibria needs for petroleum exploration and production industry," Fluid Phase Equilib., vol. 84, p. 343, 1993.

[13] S. Srinivasan and M. Z. Saghir, "Thermodiffusion in multicomponent mixtures: Thermodynamic, algebraic, and neuro-computing models," SpringerBriefs in Applied Sciences and Technology, 2013.

[14] I. Morozov K, "The soret effect in liquid mixturesA review," J. Non-Equilib. Thermodyn., vol. 41, p. 151, 2016.

[15] S. Srinivasan, "Measurements on thermodiffusion in ternary hydrocarbon mixtures at high pressure," J. Chem. Phys., vol. 131, p. 124508, 2009.

[16] S. Antoun, M. Z. Saghir, and S. Srinivasan, "Composition effect on thermophobicity of ternary mixtures: An enhanced molecular dynamics method," The Journal of Chemical Physics, jul 2018.

[17] H. Machrafi and G. Lebon, "The role of several heat transfer mechanisms on the enhancement of thermal conductivity in nanofluids," Continuum Mechanics and Thermodynamics, vol. 28, no. 5, pp. 1461-1475, 2016.

[18] N. Ali, J. A. Teixeira, and A. Addali, "A Review on Nanofluids: Fabrication, Stability, and Thermophysical Properties," Journal of Nanomaterials, 2018. 
[19] R. S. Vajjha and D. K. Das, "A review and analysis on influence of temperature and concentration of nanofluids on thermophysical properties, heat transfer and pumping power," International Journal of Heat and Mass Transfer, 2012.

[20] S. K. Das, S. U. Choi, W. Yu, and T. Pradeep, Nanofluids: Science and Technology. 2007.

[21] J. J. Wang, R. T. Zheng, J. W. Gao, and G. Chen, "Heat conduction mechanisms in nanofluids and suspensions," 2012.

[22] V. Bianco, O. Manca, S. Nardini, and K. Vafai, Heat transfer enhancement with nanofluids. 2015.

[23] R. V. Pinto and F. A. S. Fiorelli, "Review of the mechanisms responsible for heat transfer enhancement using nanofluids," 2016.

[24] M. Bahiraei, "Particle migration in nanofluids: A critical review," 2016.

[25] J. Mui, J. Ngo, and B. Kim, "Aggregation and Colloidal Stability of Commercially Available $\mathrm{Al}_{2} \mathrm{O}_{3}$ Nanoparticles in Aqueous Environments," Nanomaterials, 2016.

[26] K. Ramachandran, K. Kadirgama, O. I. Awad, D. Ramasamy, M. Samykano, and W. H. Azmi, "Comprehensive review of principle factors for thermal conductivity and dynamic viscosity enhancement in thermal transport applications: An analytical tool approach," International Communications in Heat and Mass Transfer, 2018.

[27] N. Bouguerra, S. Poncet, and S. Elkoun, "Dispersion regimes in alumina/water-based nanofluids: Simultaneous measurements of thermal conductivity and dynamic viscosity," International Communications in Heat and Mass Transfer, vol. 92, pp. 51-55, 2018.

[28] F. Jabbari, A. Rajabpour, and S. Saedodin, "Thermal conductivity and viscosity of nanofluids: A review of recent molecular dynamics studies," 2017.

[29] M. P. Allen and D. J. Tildesley, J Computer Simulation of Liquids. 1987.

[30] S. Plimpton, P. Crozier, and A. Thompson, "LAMMPS-large-scale atomic/molecular massively parallel simulator," Sandia National Laboratories, 2007. 
[31] M. Horodecki and J. Oppenheim, "Fundamental limitations for quantum and nanoscale thermodynamics," Nature Communications, 2013.

[32] D. J. Evans and G. Morriss, Statistical mechanics of nonequilibrium liquids, second edition. 2008.

[33] G. Lebon, D. Jou, and J. Casas-Vazquez, Understanding Non-equilibrium Thermodynamics. 2008.

[34] R. W. Hockney and J. W. Eastwood, Computer Simulation Using Particles. 2004.

[35] J. P. Ewen, C. Gattinoni, F. M. Thakkar, N. Morgan, H. A. Spikes, and D. Dini, "A comparison of classical force-fields for molecular dynamics simulations of lubricants," Materials, vol. 9, p. 651, 2016.

[36] de Groot SR and P. O. Mazur, Non-Equilibrium Thermodynamics, vol. 16. 1964.

[37] L. Onsager, "Reciprocal relations in irreversible processes. I.," Physical Review, 1931.

[38] B. Hafskjold, T. Ikeshoji, and S. Kjelstrup Ratkje, "On the molecular mechanism of thermal diffusion in liquids," Molecular Physics, 1993.

[39] O. Minster, "Thermodiffusion in multicomponent n-alkane mixtures," NPJ Microgravity, vol. 3, p. 1, 2017.

[40] Y. Demirel and S. I. Sandler, "Linear-nonequilibrium thermodynamics theory for coupled heat and mass transport," International Journal of Heat and Mass Transfer, 2001.

[41] L. Martinez, R. Andrade, E. G. Birgin, J. M. Martinez, and M. Martinez J, "PACKMOL: A package for building initial configurations for molecular dynamics simulations," Journal of Computational Chemistry, vol. 30, p. 2157, 2009.

[42] S. Kjelstrup, D. Bedeaux, I. Inzoli, and J. M. Simon, "Criteria for validity of thermodynamic equations from non-equilibrium molecular dynamics simulations," Energy, vol. 33, p. 1185, 2008.

[43] F. Muller-Plathe, "Reverse nonequilibrium molecular-dynamics calculation of the Soret coefficient in liquid benzene/cyclohexane mixtures," J. Phys. Chem., vol. 123, p. 124502, 2005. 
[44] S. Wiegand, "Soret and mass diffusion measurements and molecular dynamics simulations of n-pentanen-decane mixtures," J. Phys. Chem., vol. 116, p. 3718, 2002.

[45] G. Leaist D, J. Chan, J. J. Popov, S. Kolisnek-Kehl, and D. G. Leaist, "Soret coefficients for aqueous polyethylene glycol solutions and some tests of the segmental model of polymer thermal diffusion," J. Solution Chem., vol. 32, p. 197, 2003.

[46] F. Huang, P. Chakraborty, C. C. Lundstrom, C. Holmden, J. J. Glessner, S. W. Kieffer, C. E. Lesher, and F. Huang, "Isotope fractionation in silicate melts by thermal diffusion," Nature, vol. 464, p. 396, 2010.

[47] S. Kjelstrup, "Thermal phenomena associated with water transport across a fuel cell membrane: Soret and Dufour effects," J. Membr. Sci., vol. 431, p. 96, 2013.

[48] K. Liu, "Analysis for high-order effects in thermal lagging to thermal responses in biological tissue," Int. J. Heat Mass Transfer, vol. 81, p. 347, 2015.

[49] A. Libchaber, "Trapping of DNA by thermophoretic depletion and convection," Phys. Rev. Lett., vol. 89, p. 188103, 2002.

[50] S. Srinivasan and M. Z. Saghir, Experimental approaches to study thermodiffusion - A review, vol. 50. 2011.

[51] J. K. Platten, "The Soret Effect: A Review of Recent Experimental Results," Journal of Applied Mechanics, 2006.

[52] A. Parsa, S. Srinivasan, and M. Z. Saghir, "Impact of density gradients on the fluid flow inside a vibrating cavity subjected to soret effect," Canadian Journal of Chemical Engineering, 2013.

[53] M. Z. Saghir and M. Eslamian, "A critical review of thermodiffusion models: Role and significance of the heat of transport and the activation energy of viscous flow," Journal of Non-Equilibrium Thermodynamics, vol. 34, p. 97, 2009.

[54] B. Rousseau, "Isotopic Soret effect in ternary mixtures: Theoretical predictions and molecular simulations," J. Chem. Phys., vol. 143, p. 174503, 2015. 
[55] P. Polyakov and S. Wiegand, "Systematic study of the thermal diffusion in associated mixtures," Journal of Chemical Physics, 2008.

[56] G. Galliero, B. Duguay, J. P. Caltagirone, and F. Montel, "Thermal diffusion sensitivity to the molecular parameters of a binary equimolar mixture, a non-equilibrium molecular dynamics approach," Fluid Phase Equilibria, 2003.

[57] Z. Saghir M, "Thermodiffusion in binary and ternary hydrocarbon mixtures studied using a modified heat exchange algorithm," Therm. Sci. Eng. Prog., vol. 4, p. 168, 2017.

[58] C. Dellago, "An enhanced version of the heat exchange algorithm with excellent energy conservation properties," J. Phys. Chem., vol. 143, p. 124104, 2015.

[59] G. Galliero, "Equilibrium, interfacial and transport properties of n-alkanes: Towards the simplest coarse grained molecular model," Chemical Engineering Research and Design, 2014.

[60] M. G. Martin and J. I. Siepmann, "Transferable Potentials for Phase Equilibria. 1. United-Atom Description of n-Alkanes ," The Journal of Physical Chemistry B, 2002.

[61] B. Smit, S. Karabomi, and J. Ilja Siepmann, "Computer simulations of vapor-liquid phase equilibria of n-alkanes," The Journal of Chemical Physics, 1995.

[62] W. Allen and R. L. Rowley, "Predicting the viscosity of alkanes using nonequilibrium molecular dynamics: Evaluation of intermodular potential models," 1997.

[63] H. Hoang, S. Delage-Santacreu, and G. Galliero, "Simultaneous Description of Equilibrium, Interfacial, and Transport Properties of Fluids Using a Mie Chain CoarseGrained Force Field," Industrial and Engineering Chemistry Research, 2017.

[64] D. K. Dysthe, A. H. Fuchs, and B. Rousseau, "Fluid transport properties by equilibrium molecular dynamics. III. Evaluation of united atom interaction potential models for pure alkanes," J. Chem. Phys., vol. 112, p. 7581, 2000.

[65] P. Ungerer, C. Beauvais, J. Delhommelle, A. Boutin, B. Rousseau, and A. H. Fuchs, "Optimization of the anisotropic united atoms intermolecular potential for n-alkanes," Journal of Chemical Physics, 2000. 
[66] H. Zhang and J. F. Ely, "AUA model NEMD and EMD simulations of the shear viscosity of alkane and alcohol systems," Fluid Phase Equilibria, 2004.

[67] Z. A. Makrodimitri, D. J. Unruh, and I. G. Economou, "Molecular simulation of diffusion of hydrogen, carbon monoxide, and water in heavy n -alkanes," Journal of Physical Chemistry B, vol. 115, 2011.

[68] J. J. Potoff, J. R. Errington, and A. Z. Panagiotopoulos, "Molecular simulation of phase equilibria for mixtures of polar and non-polar components," Molecular Physics, vol. 97 , pp. $1073-1083,1999$.

[69] A. I. Jewett, Z. Zhuang, and J.-E. Shea, "Moltemplate a Coarse-Grained Model Assembly Tool," Biophysical Journal, 2013.

[70] B. Hafskjold and S. K. Ratkje, "Criteria for local equilibrium in a system with transport of heat and mass," Journal of Statistical Physics, 1995.

[71] G. Pranami and M. H. Lamm, "Estimating Error in Diffusion Coefficients Derived from Molecular Dynamics Simulations," Journal of Chemical Theory and Computation, 2015.

[72] W. Kohler, "Contribution to the benchmark for ternary mixtures: Measurement of the Soret and thermodiffusion coefficients of tetralin+isobutylbenzene $+n$-dodecane at a composition of $(0.8 / 0.1 / 0.1)$ mass fractions by two-color optical beam deflection," Eur. Phys. J. E, vol. 38, p. 24, 2015.

[73] J. W. Mutoru, W. Smith, C. S. O'Hern, and A. Firoozabadi, "Molecular dynamics simulations of diffusion and clustering along critical isotherms of medium-chain nalkanes," Journal of Chemical Physics, vol. 138, 2013.

[74] C. Bevington and R. Philip, "Data Reduction and Error Analysis for the Physical Sciences.," Journal of the American Statistical Association, 2006.

[75] D. Alonso De Mezquia, M. Mounir Bou-Ali, J. A. Madariaga, and C. Santamaria, "Mass effect on the Soret coefficient in n -alkane mixtures," Journal of Chemical Physics, vol. 140, 2014. 
[76] P. Blanco, P. Polyakov, M. Mounir Bou-Ali, and S. Wiegand, "Thermal diffusion and molecular diffusion values for some alkane mixtures: A comparison between thermogravitational column and thermal diffusion forced Rayleigh scattering," Journal of Physical Chemistry B, vol. 112, pp. 8340 - 8345, 2008.

[77] V. Shevtsova, "Contribution to the benchmark for ternary mixtures: Measurement of the Soret, diffusion and thermodiffusion coefficients in the ternary mixture THN/IBB/nC12 with 0.8/0.1/0.1 mass fractions in ground and orbital laboratories," Eur. Phys. J. E, vol. 38, p. 27, 2015.

[78] K. Platten J, "Contribution to the benchmark for ternary mixtures: Determination of Soret coefficients by the thermogravitational and the sliding symmetric tubes techniques," Eur. Phys. J. E, vol. 38, p. 28, 2015.

[79] S. Saghir M, "Contribution to the benchmark for ternary mixtures: Transient analysis in microgravity conditions," Eur. Phys. J. E, vol. 38, p. 25, 2015.

[80] V. Vaerenbergh S, "Contribution to the benchmark for ternary mixtures: Measurement of diffusion and Soret coefficients of ternary system tetrahydronaphtaleneisobutylbenzene-n-dodecane with mass fractions 80-10-10 at 25C," Eur. Phys. J. E, vol. 38 , p. 26, 2015.

[81] P. Lyubimova T, "Contribution to the benchmark for ternary mixtures: Measurement of diffusion and Soret coefficients in 1,2,3,4-tetrahydronaphthalene, isobutylbenzene, and dodecane onboard the ISS," Eur. Phys. J. E, vol. 38, p. 29, 2015.

[82] S. Van Varenbergh, "Benchmark values for the soret, thermodiffusion and molecular diffusion coefficients of the ternary mixture tetralin + isobutylbenzene $+n$-dodecane with 0.8-0.1-0.1 mass fraction," Eur. Phys. J. E, vol. 38, p. 30, 2015.

[83] Z. Saghir M, "Thermodiffusion in ternary hydrocarbon mixtures. Part 1: ndodecane/isobutylbenzene/tetralin," J. Non-Equilib. Thermodyn., vol. 36, p. 243, 2011.

[84] Z. Saghir M, "Significance of equation of state and viscosity on the thermodiffusion coefficients of a ternary hydrocarbon mixture," High Temp. - High Pressures, vol. 39, p. $65,2010$. 
[85] Z. Saghir M, "Dynamic thermodiffusion theory for ternary liquid mixtures," J. NonEquilib. Thermodyn., vol. 35, p. 51, 2010.

[86] M. Bou-Ali M, "Investigation of the soret effect in binary, ternary and quaternary hydrocarbon mixtures: New expressions for thermodiffusion factors in quaternary mixtures," Int. J. Therm. Sci., vol. 49, p. 2128, 2010.

[87] G. Galliero, S. Srinivasan, and M. Z. Saghir, "Estimation of thermodiffusion in ternary alkane mixtures using molecular dynamics simulations and an irreversible thermodynamic theory," High Temperatures - High Pressures, 2009.

[88] B. Anders S, "The national institute of standards and technology," CPA J., vol. 86, p. $72,2016$.

[89] G. Sadowski, "Thermophobicity of liquids: Heats of transport in mixtures as pure component properties," Phys. Rev. Lett., vol. 109, p. 65901, 2012.

[90] S. Antoun and M. Ziad Saghir, "Thermophysical Properties and Heat Transfer Enhancement of Alumina Nanofluid: An Improved Molecular Dynamics Study,"

[91] M. Chandrasekar, S. Suresh, and A. Chandra Bose, "Experimental investigations and theoretical determination of thermal conductivity and viscosity of $\mathrm{Al}_{2} \mathrm{O}_{3}$ /water nanofluid," Experimental Thermal and Fluid Science, 2010.

[92] X. Wang, X. Xu, and S. U. S. Choi, "Thermal Conductivity of Nanoparticle - Fluid Mixture," Journal of Thermophysics and Heat Transfer, 2008.

[93] C. T. Nguyen, F. Desgranges, N. Galanis, G. Roy, T. Mare, S. Boucher, and H. Angue Mintsa, "Viscosity data for $\mathrm{Al}_{2} \mathrm{O}_{3}$-water nanofluid-hysteresis: is heat transfer enhancement using nanofluids reliable?," International Journal of Thermal Sciences, 2008.

[94] E. B. Elcioglu, A. Guvenc Yazicioglu, A. Turgut, and A. S. Anagun, "Experimental study and Taguchi Analysis on alumina-water nanofluid viscosity," Applied Thermal Engineering, 2018.

[95] M. P. Beck, Y. Yuan, P. Warrier, and A. S. Teja, "The effect of particle size on the thermal conductivity of alumina nanofluids," Journal of Nanoparticle Research, 2009. 
[96] E. V. Timofeeva, A. N. Gavrilov, J. M. McCloskey, Y. V. Tolmachev, S. Sprunt, L. M. Lopatina, and J. V. Selinger, "Thermal conductivity and particle agglomeration in alumina nanofluids: Experiment and theory," Physical Review E - Statistical, Nonlinear, and Soft Matter Physics, 2007.

[97] K. Bashirnezhad, S. Bazri, M. R. Safaei, M. Goodarzi, M. Dahari, O. Mahian, A. S. Dalkiliça, and S. Wongwises, "Viscosity of nanofluids: A review of recent experimental studies," International Communications in Heat and Mass Transfer, 2016.

[98] D. Lee, J. W. Kim, and B. G. Kim, "A new parameter to control heat transport in nanofluids: Surface charge state of the particle in suspension," Journal of Physical Chemistry B, 2006.

[99] S. Ghosh, H. Mashayekhi, P. Bhowmik, and B. Xing, "Colloidal stability of $\mathrm{Al}_{2} \mathrm{O}_{3}$ nanoparticles as affected by coating of structurally different humic acids," Langmuir, 2010.

[100] G. Lefevre, M. Duc, and M. Fedoroff, "Effect of solubility on the determination of the protonable surface site density of oxyhydroxides," Journal of Colloid and Interface Science, 2004.

[101] M. Delhorme, C. Labbez, C. Caillet, and F. Thomas, "Acid-base properties of 2:1 clays. I. modeling the role of electrostatics," Langmuir, 2010.

[102] F. Loosli, L. Vitorazi, J. F. Berret, and S. Stoll, "Towards a better understanding on agglomeration mechanisms and thermodynamic properties of $\mathrm{TiO}_{2}$ nanoparticles interacting with natural organic matter," Water Research, 2015.

[103] Z. Jia-Fei, L. Zhong-Yang, N. Ming-Jiang, and C. Ke-Fa, "Dependence of nanofluid viscosity on particle size and pH value," Chinese Physics Letters, 2009.

[104] B. C.A.S., L. R.G., and K. N.A., "Nonadditivity of nanoparticle interactions," Science, 2015.

[105] Y. Min, M. Akbulut, K. Kristiansen, Y. Golan, and J. Israelachvili, "The role of interparticle and external forces in nanoparticle assembly," Nature Materials, 2008. 
[106] R. Wang, S. Qian, and Z. Zhang, "Investigation of the aggregation morphology of nanoparticle on the thermal conductivity of nanofluid by molecular dynamics simulations," International Journal of Heat and Mass Transfer, 2018.

[107] J. P. Meyer, S. A. Adio, M. Sharifpur, and P. N. Nwosu, "The Viscosity of Nanofluids: A Review of the Theoretical, Empirical, and Numerical Models," 2016.

[108] W. Q. Lu and Q. M. Fan, "Study for the particle's scale effect on some thermophysical properties of nanofluids by a simplified molecular dynamics method," Engineering Analysis with Boundary Elements, 2008.

[109] G. Galliero and S. Volz, "Thermodiffusion in model nanofluids by molecular dynamics simulations," Journal of Chemical Physics, 2008.

[110] C. Hu, M. Bai, J. Lv, P. Wang, and X. Li, "Molecular dynamics simulation on the friction properties of nanofluids confined by idealized surfaces," Tribology International, 2014.

[111] S. L. Lee, R. Saidur, M. F. Sabri, and T. K. Min, "Molecular dynamic simulation on the thermal conductivity of nanofluids in aggregated and non-aggregated states," Numerical Heat Transfer; Part A: Applications, 2015.

[112] M. Sedighi and A. Mohebbi, "Investigation of nanoparticle aggregation effect on thermal properties of nanofluid by a combined equilibrium and non-equilibrium molecular dynamics simulation," Journal of Molecular Liquids, 2014.

[113] H. Babaei, P. Keblinski, and J. M. Khodadadi, "Equilibrium molecular dynamics determination of thermal conductivity for multi-component systems," in Journal of Applied Physics, 2012.

[114] A. B. Rahane, M. D. Deshpande, and V. Kumar, "Structural and electronic properties of $\left(\mathrm{Al}_{2} \mathrm{O}_{3}\right) \mathrm{n}$ clusters with $\mathrm{n}=1-10$ from first principles calculations," Journal of Physical Chemistry C, 2011.

[115] J. D. Kubicki, "Molecular cluster models of aluminum oxide and aluminum hydroxide surfaces," American Mineralogist, 1998. 
[116] M. Lagauche, K. Larmier, E. Jolimaitre, K. Barthelet, C. Chizallet, L. Favergeon, and M. Pijolat, "Thermodynamic Characterization of the Hydroxyl Group on the $\gamma$ Alumina Surface by the Energy Distribution Function," Journal of Physical Chemistry C, 2017.

[117] R. Wischert, P. Laurent, C. Coperet, F. Delbecq, and P. Sautet, " $\gamma$-Alumina: The essential and unexpected role of water for the structure, stability, and reactivity of "defect" sites," Journal of the American Chemical Society, 2012.

[118] R. Reocreux, T. Jiang, M. Iannuzzi, C. Michel, and P. Sautet, "Structuration and Dynamics of Interfacial Liquid Water at Hydrated $\gamma$-Alumina Determined by ab Initio Molecular Simulations: Implications for Nanoparticle Stability," ACS Applied Nano Materials, 2018.

[119] Z. Lou and M. Yang, "Molecular dynamics simulations on the shear viscosity of $\mathrm{Al}_{2} \mathrm{O}_{3}$ nanofluids," Computers and Fluids, 2015.

[120] San Diego: Accelrys Software Inc., "Discovery Studio Modeling Environment, Release $4.5, " 2015$.

[121] H. J. Berendsen, J. R. Grigera, and T. P. Straatsma, "The missing term in effective pair potentials," Journal of Physical Chemistry, 1987.

[122] M. Sedighi and A. Mohebbi, "Investigation of nanoparticle aggregation effect on thermal properties of nanofluid by a combined equilibrium and non-equilibrium molecular dynamics simulation," Journal of Molecular Liquids, 2014.

[123] M. S. Patil, J. H. Seo, S. J. Kang, and M. Y. Lee, "Review on synthesis, thermophysical property, and heat transfer mechanism of nanofluids," 2016.

[124] A. Lenert, Y. Nam, and E. N. Wang, "HEAT TRANSFER FLUIDS," Annual Review of Heat Transfer, 2012. 
